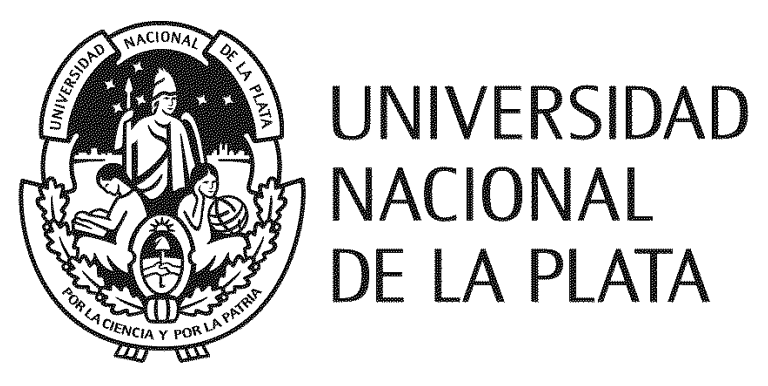

FACULTAD DE CIENCIAS EXACTAS; DEPARTAMENTO DE QUÍMICA

TESIS PRESENTADA PARA OPTAR AL GRADO DE DOCTOR DE LA FACULTAD DE CIENCIAS EXACTAS

\title{
ESTUDIO DE LAS PROPIEDADES \\ FISICOQUÍMICAS DE NANOCANALES DE ESTADO SÓLIDO MODIFICADOS CON POLIELECTROLITOS
}

Lic. Facundo Matías Gilles

Director: Dr. Omar Azzaroni

Co-director: Dr. Igal Szleifer 


\section{Agradecimientos}

Esta tesis representa el final de mi trabajo para obtener el grado de doctor en ciencias, título que representa la culminación de un sueño del chico de 12 años que alguna vez fui. Aunque soy el autor del trabajo, de los resultados y del escrito, todo esto no hubiese sido posible sin un contexto favorable para su desarrollo. Ese contexto complejo por su propia naturaleza involucra aspectos técnicos/científicos, aspectos personales/emocionales y aspectos socio-políticos, que se mezclan e interfieren entre sí.

Fueron imprescindibles mis mentores Omar e Igal, porque durante estos años me han guiado, acompañado y aconsejado, tanto en lo profesional como en lo personal. Agradezco especialmente a Mario Tagliazucchi quién desde el desafortunado incidente que sufrió Igal, ha asumido un rol fundamental para la generación y comprobación de los resultados de esta tesis, así como para la corrección del escrito final. Las discusiones que hemos tenido respecto de la teoría, el código fuente y la ciencia en general fueron y son de gran valor para mí. Quiero agradecer a todo el equipo de trabajo del Laboratorio de Materia Blanda, donde he desarrollado mis actividades. A los investigadores, Walde, Lore y Juan, porque me acompañaron y me alentaron para que termine este proyecto. A los que estuvieron antes que yo, Apu y Marcos, y los integrantes que se fueron sumando después: Agus Lorentz, Esteban, Gonza PM, Gonza F, Nico M., Juan A., Gustavo, Seba A., Jime T., Eliana M., Giya D.. A Alberto Albesa, «Beto», por las discusiones científicas, por la amistad y por su ayuda en los momentos difíciles. A Mariana T. por sus consejos durante los últimos meses y por haberme ayudado en la corrección del texto. También quiero agradecer a todos los integrantes del grupo de Modelado Molecular de Biomateriales, por que hicieron agradable mi estadía en Chicago.

En los aspectos personales/emocionales, agradezco a mis padres porque ellos me dieron dos herramientas que considero valiosas: la tenacidad y el optimismo (aunque algunas veces fui un tenaz pesimista y otras un optimista inconstante, me sigo esforzando). A mi hermana porque siempre estuvo atenta a su hermano «el científico» (con todas las cosas malas de tener un hermano científico), para ella también van mis agradecimientos. A mis amigos de siempre Gonza, Leo y Renzo, la «familia macana»: Ariel, Damián, Cynthia y Javi. Quiero agradecer a Brenda B. por sus consejos certeros, por su escucha activa, por su bendición de dragón.

Finalmente, debo agradecer al Estado argentino por financiar mi educación desde el nivel primario hasta el posgrado. A las instituciones del Estado argentino: el CONICET, institución que me ha beneficiado con una beca de doctorado, cuyo estipendio me ha permitido vivir dignamente, la UNLP y el INIFTA por aceptar el plan de trabajo y brindarme un lugar de trabajo limpio, seguro y confortable. Gracias a la comunidad científica en particular y la sociedad argentina en general por ser los benefactores de mi formación y mi trabajo. Espero poder retribuir con mis esfuerzos las oportunidades que me han dado. A continuación los frutos... 
"La verdad surge más fácilmente del error que de la confusión."

Francis Bacon 


\section{Índice general}

$\begin{array}{lr}\text { 1. Introducción } & \mathbf{5}\end{array}$

1.1. Antecedentes . . . . . . . . . . . . . . . . . . . 6

1.2. Interacciones Moleculares . . . . . . . . . . . . . . . . . . . 9

1.3. Teoría en nanocanales . . . . . . . . . . . . . . . . . . . 11

1.4. Sobre este Trabajo . . . . . . . . . . . . . . . . . . . . 12

2. Enfoque Teórico $r$

2.1. Equilibrio Químico . . . . . . . . . . . . . . . . . . . . 14

2.2. Teoría Molecular . . . . . . . . . . . . . . . . . . . 17

2.2.1. Contribuciones Entrópicas . . . . . . . . . . . . . 18

2.2.2. Interacciones Intermoleculares . . . . . . . . . . . . . . . . . . . 22

2.2.3. Intercambio de Partículas con el Entorno: Ensamble Gran Canónico . . . 23

2.2.4. Restricciones a la Energía Libre: Empaquetamiento, Electroneutralidad y Normalización de la Probabilidad de Configuraciones $P(\alpha)$. . . . . . . 24

2.3. Optimización Analítica . . . . . . . . . . . . . . . . . . 27

2.3.1. Expresión para la Energía Reducida . . . . . . . . . . . . . . 32

3. Propiedades de Transporte $\quad 33$

3.1. Conductancia $(G) \ldots \ldots \ldots \ldots$. . . . . . . . . . . . . . . . . . . . . . . . . .

3.1.1. Conductancia para distintas geometrías . . . . . . . . . . . 35

4. Adsorción $\quad 42$

4.1. Energía libre del sistema . . . . . . . . . . . . . . . . . . . . . . 43

4.2. Adsorción en nanocanales cilíndricos . . . . . . . . . . . . . . . . 47

5. Brushes Polipróticos $\quad 55$

5.1. Conductancia de Nanocanales . . . . . . . . . . . . . . . . . . . . 57

5.2. Nanocanales Cilíndricos . . . . . . . . . . . . . . . . . . 58

5.3. Nanocanales No Cilíndricos . . . . . . . . . . . . . . . . . . 65

$\begin{array}{lr}\text { 6. Conclusiones } & 68\end{array}$

$\begin{array}{lr}\text { Bibliografía } & 71\end{array}$

$\begin{array}{ll}\text { A. Tablas de Símbolos y Valores } & 77\end{array}$

$\begin{array}{lr}\text { B. Soluciones de Bulk } & \mathbf{8 0}\end{array}$

B.0.1. Simetría en las ecuaciones del grado de disociación . . . . . . . . . . . 81 
B.1. Relaciones entre las variables de bulk experimentales y las variables de entrada en la teoría . . . . . . . . . . . . . . . . . . . . . 82

C. Modelo de Capa Polimérica Homogénea $\quad 83$

$\begin{array}{lr}\text { D. Discretización } & \mathbf{8 8}\end{array}$

D.1. Ecuación de Poisson . . . . . . . . . . . . . . . . . . . . . . . 88

D.2. Densidad de probabilidad $P(\alpha) \ldots \ldots \ldots \ldots$. . . . . . . . . . 90

$\begin{array}{ll}\text { E. Aproximación de Campo Medio } & 91\end{array}$

$\begin{array}{lr}\text { F. Método Variacional } & 94\end{array}$ 


\section{Capítulo 1}

\section{Introducción}

Durante el siglo pasado la física ha avanzado en tres grandes frentes: lo grande, lo pequeño y lo complejo. Mientras que el estudio de lo grande y de lo pequeño (aquello que concierne la evolución del universo como un todo y aquello que concierne a la estructura subatómica de la materia, respectivamente), involucra el descubrimiento de las leyes fundamentales de la naturaleza, el estudio de lo complejo implica comprender como se combinan las leyes de la naturaleza conocidas. Haciendo un paralelismo con la biología moderna que ha evolucionado desde el estudio de organismos unicelulares simples (como las bacterias) hacia el estudio de organismos complejos pluricelulares (como las plantas, los invertebrados y vertebrados), durante la segunda mitad del siglo XX la física ha evolucionado desde el estudio de las interacciones entre partículas hacia el estudio de fluidos complejos.

Los fluidos complejos tienen localmente la mobilidad de líquidos pero están constituidos por polímeros, surfactantes, cristales líquidos, coloides, etc; Algunos ejemplos podrían ser: la mayonesa, la crema de afeitar o el gel para el pelo. En general, estos fluidos complejos se denominan con el nombre de materia blanda. En 1991 durante la ceremonia de entrega de su premio Nobel, Pierre-Gilles de Gennes[20], quién forjó el concepto materia blanda, comentó en su discurso el caso de indígenas de la cuenca del amazonas que construían una especie de botas a partir de la savia del árbol Hevea brasiliensis (más comúnmente llamado árbol del caucho). El procedimiento consiste en untar la savia sobre los pies y dejar secar durante un tiempo relativamente corto para obtener unas botas. Desde el punto de vista microscópico al comienzo se tiene un conjunto de cadenas poliméricas flexibles todas independientes entre sí. Luego el oxígeno del aire forma enlaces entre las cadenas lo que trae aparejado un cambio macroscópico sorprendente: el pasaje de un líquido a una red estructurada capaz de resistir tensiones, material que denominamos caucho. Este es un ejemplo de sistema de materia blanda porque frente a pequeños cambios químicos, el material responde con notables cambios macroscópicos. En la actualidad el concepto de materia blanda engloba una infinidad de sistemas tanto sintéticos como biológicos. Una definición de materia blanda debe incluir que las escalas espaciales relevantes son del orden de los nanómetros y las energías son del orden de la energía térmica $\left(\sim k_{B} T\right)$, además las propiedades más interesantes de los sistemas de materia blanda resultan de un sutil balance entre la energía y la entropía. Expliquemos esto con un ejemplo clásico: las interacciones entálpicas entre las moléculas de polímeros que se encuentran entrecruzadas en el material de una banda elástica, dependen fuertemente de la configuración de las cadenas, y las propiedades elásticas se deben casi enteramente a los cambios en la entropía configuracional de las cadenas estiradas. Por ejemplo, si se considera una cadena polimérica ideal y se la estira hasta una longitud determinada, el trabajo realizado es igual al trabajo que se hubiera hecho si 
se confinaba la cadena ideal (por ejemplo presionándola sobre una superficie) hasta obtener la misma longitud[24]. Los efectos entrópicos y de confinamiento aparecen en simultáneo a través de la imposición de una interfaz o un confinamiento geométrico, que disminuye los grados de libertad a la vez que incorpora nuevas interacciones moleculares. Estos fenómenos pueden tener múltiples consecuencias, por ejemplo el desplazamiento del equilibrio hacia estructuras más ordenadas.

Los sistemas cargados en medios acuosos también derivan muchas de sus propiedades de los efectos entrópicos y de confinamiento. Por ejemplo, las repulsiones entre dos superficies de igual carga separadas por una solución iónica puede comprenderse en términos de la presión osmótica que proviene del confinamiento de contraiones en el espacio entre las placas[53]. De la misma forma la adsorción de polielectrolitos sobre una superficie cargada proviene no de la interacción electrostática entre el polielectrolito y la superficie, sino de la ganancia entrópica de los contraiones que se liberan al seno de la solución mientras la cadena es confinada/adsorbida sobre la superficie[39].

Menos de tres décadas después de "la fundación" del concepto de materia blanda, la posibilidad de construir sistemas con materia blanda en confinamiento surge como una estrategia para el desarrollo de micro y nanotecnología. Por ejemplo, la construcción de nanocanales de estado sólido, permite estudiar sistemas de materia blanda en nanoconfinamiento, con potenciales aplicaciones en biosensado o secuenciación de ADN. Debemos distinguir entre materiales blandos cuyas escalas espaciales se encuentran en el orden del micrón $\left(10^{-6} \mathrm{~m}\right)$ como pueden ser los coloides y aquellos cuyas escalas espaciales son las dimensiones moleculares $\left(10^{-9} \mathrm{~m}\right)$ : soluciones poliméricas, surfactantes, cristales líquidos. En estos últimos las escalas espaciales son nanoscópicas y es allí donde el nanoconfinamiento compite con las interacciones intermoleculares.

\subsection{Antecedentes e hipótesis derivadas de los mismos}

Tipos de nanocanales y poros: La confección de nanocanales y nanoporos modificados químicamente abarca varias tecnologías y materiales que comparten ciertas características esenciales.

1. Es posible construir membranas con multiporos o con un único poro cuyos diámetros se encuentran entre 1 y $1000 \mathrm{~nm}$.

2. Pueden ser modificados químicamente de forma tal de otorgar funcionalidad y sensibilidad a estímulos externos.

3. Sirven como medio para el transporte selectivo en distintas aplicaciones (filtración, separación, sensado).

Ejemplos de sistemas que cumplen estas características son: canales de estado sólido construidos con haces de iones o electrones (ion/electron sculpting), películas nanoporosas coloidales, materiales mesoporosos, nanopipetas de vidrio y membranas poliméricas "track-etched". Debido al control que puede lograrse en el tamaño y la forma del nanocanal, la técnica de track-etched es una de las más utilizadas para la confección de nanocanales. Esta técnica consiste en preparar las membranas por irradiación con iones pesados de alta energía (como por ejemplo uranio y bismuto). Debido a su alta energía estos iones no son detenidos por la membrana y la atraviesan completamente, produciendo una degradación de la membrana en su trayectoria. La zona debilitada es tratada químicamente (proceso de "etching") para producir nanocanales cuya longitud 


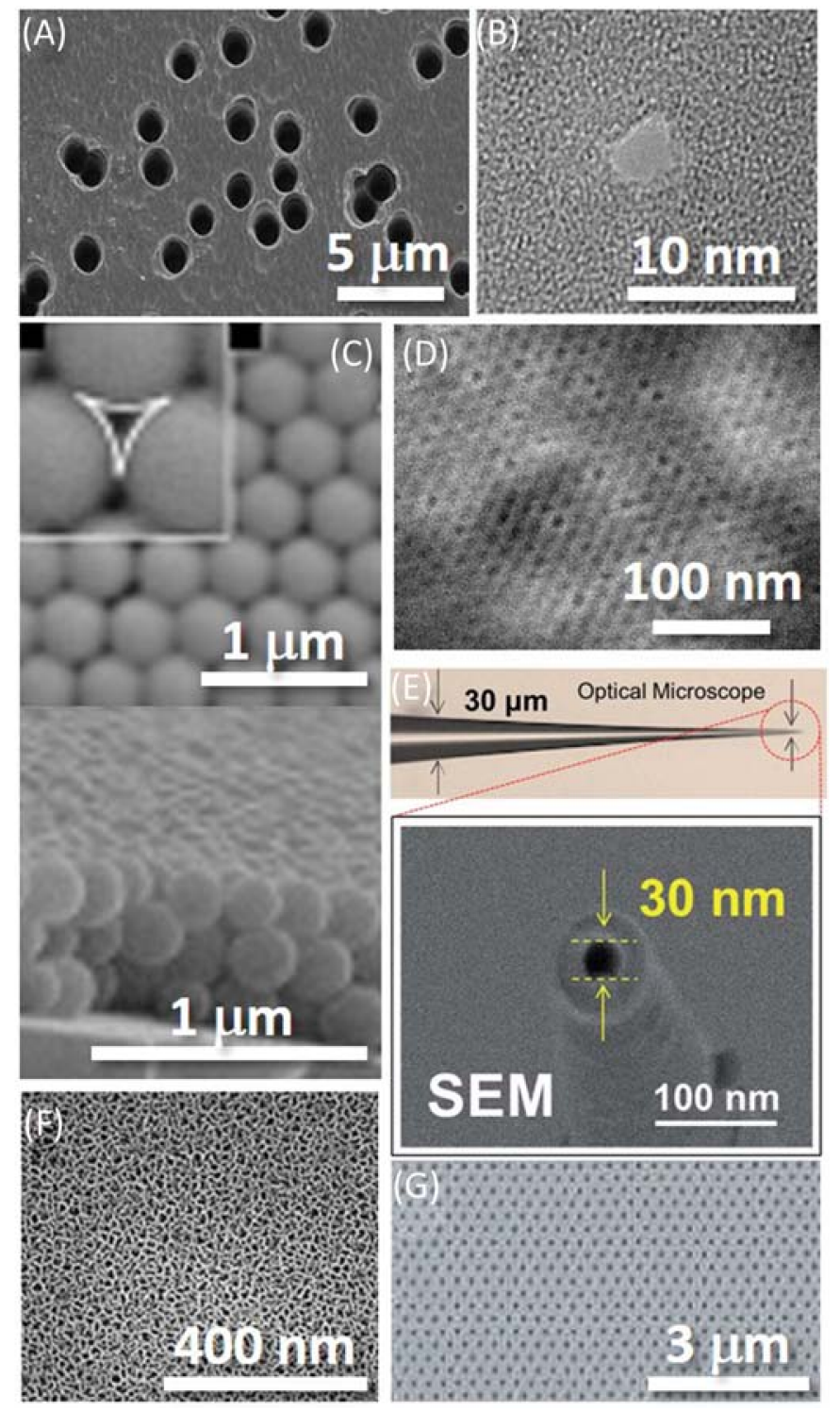

Figura 1.1.1: Imágenes de microscopía para distintos tipos de nanocanales: (A) Microscopía electrónica de barrido (SEM) de nanocanales construidos en membranas de (PET) con la técnica de track-etched; (B) Microscopía electrónica de transmisión (TEM) de una membrana ultra fina de óxido de silicio con un único nanoporo; (C) Imágen SEM de una membrana coloidal porosa (panel superior: vista superior, panel inferior: vista lateral, el detalle en el panel superior muestra que los nanoporos están formados por los espacios en la estructura fcc del arreglo coloidal); (D) Imagen SEM de una membrana mesoporosa de silica; (E) Imagen óptica (panel superior) e imagen SEM (panel inferior) de una nanopipeta de borosilicato; (F) Imagen SEM de una membrana microporosa de silicona; $(\mathrm{G})$ Imagen SEM de un nanocanal en una membrana de alumina. La imagen fue tomada de la rer. : Mario Tagliazucchi Igal Szleifer, ed., Chemically Modified Nanopores and Nanochannels (Elsevier, 2016). A su vez las imágenes fueron adaptadas de las siguientes fuentes: (A) Wen, L.; Tian, Y.; Ma, J.; Zhai, J.; Jiang, L., Construction of Biomimetic Smart Nanochannels with Polymer Membranes and Application in Energy Conversion Systems. Phys. Chem. Chem. Phys. 2012, 14, 4027À4042, (B) Storm, A.J.; Chen, J.H.; Ling, X.S.; Zandbergen, H.W.; Dekker, C., Fabrication of Solid-State Nanopores with Single-Nanometre Precision. Nat. Mater. 2003, 2, 537À540, (E) An, S.; Stambaugh, C.; Kim, G.; Lee, M.; Kim, Y.; Lee, K., et al., Low-Volume Liquid Delivery and Nanolithography Using a Nanopipette Combined with a Quartz Tuning Fork-Atomic Force Microscope. Nanoscale 2012, 4, 6493À6500, (F) Velleman, L.; Shearer, C.J.; Ellis, A.V.; Losic, D.; Voelcker, N.H.; Shapter, J.G., Fabrication of Self- Supporting Porous Silicon Membranes and Tuning Transport Properties by Surface Functionalization. Nanoscale 2010, 2, 1756À1761, y (G) Lee, W.; Ji, R.; Gosele, U.; Nielsch, K., Fast Fabrication of Long-Range Ordered Porous Alumina Membranes by Hard Anodization. Nat. Mater. 2006, 5, 741À747; (D) G. Soler-Illia's Lab. 
es más grande que los diámetros internos $[63,12]$. El proceso de etching puede ser controlado para producir nanocanales con distintas geometrías (cilíndrica[79], cónica[80], con forma de cigarro[4], etc).

Las membranas multiporo se utilizan para filtrado y están disponibles comercialmente, por ejemplo, Whatman (Nucleopore and Cyclopore) y PC, comercializan membranas de policarbonato (PC). Iteip (ipPore) comercializa membranas de PC, polyamida (PI) y poliester. La técnica de bombardeo con iones pesados, por su parte, permite detener el flujo de iones luego del primer ion impactado sobre la membrana. Esto permite la fabricación de membranas con un único canal pasante[14].

La modificación química La funcionalización química de los nanocanales es habitualmente un proceso químico de múltiples pasos en donde la primera reacción química introduce en la superficie interna del nanocanal los grupos funcionales a partir de los cuales se podrán anclar las moléculas de interés. La funcionalización de la superficie es necesaria para obtener las propiedades deseadas en la pared interna del nanocanal, tales como propiedades de mojado (wetting), carga superficial, sensibilidad ante estímulos externos, actividad catalítica o afinidad biológica.

Los materiales típicos de las membranas poliméricas que se utilizan para la confección de nanocanales son: poli(tereftalato de etileno) (PET), poli-imida (PI) y policarbonato (PC). Estos polímeros contienen grupos éster, imidas y carbonatos, respectivamente, que pueden hidrolizarse durante el proceso de etching, dejando diferentes grupos funcionales sobre la superficie. Por ejemplo, la superficies de PET hidrolizadas exhiben grupos carboxilatos y grupos alcohol[19], las membranas de PI hidrolizadas exhiben grupos amina y grupos carboxylatos [58, 54] (estos últimos se encuentran en equilibrio con los anhídridos carboxilicos[54]), y las membranas de $\mathrm{PC}$ se hidrolizan a grupos fenoxi con perdidas de $\mathrm{CO}_{2}[19]$. Estos grupos formados durante el proceso de etching, determinan la reactividad química y la carga superficial de la membrana. En el caso de las membranas de PET y PI, los grupos carboxilatos de la superficie tienen una constante de equilibrio ácido-base $p K_{a} \sim 5$, por lo que para $p H>3$ estas membranas se encuentran cargadas negativamente[59]. La máxima densidad superficial de grupos carboxilatos reportada en membranas de PET es 1,5 grupos $/ n m^{2}[57]$.

Las modificaciones químicas sobre membranas track-etched de PET y PI, generalmente involucra la funcionalización de los grupos carboxilatos con una amina en solución usando la química de la carbodiimida[37, 65]. Diferentes moléculas con un grupo amina han sido utilizados en la literatura: enzimas[5], lisinas[4], iniciadores de polimerización[79] (los cuales permiten el crecimiento de brushes poliméricos en la pared interna del canal), oligómeros de ADN con grupos terminales amino[34, 35] y aminas sustituidas con ácidos fosfóricos[1], terpiridinas[2], carbohidratos[3], alkinos[64], ciclodextrinas[30], etc.

Una alternativa para funcionalizar las membranas está basada en la generación de radicales en la superficie de la membrana polimérica por exposición a plasma. Se introducen los monómeros del polímero que se quiere utilizar en una cámara de plasma para la formación de brushes poliméricos vía polimerización por radicales libres[35, 36, 81]. Esta metodología puede ser utilizada para modificar con polímeros distintos los dos lados de una membrana de PET con un único nanocanal[81,36].

Modificaciones no-covalentes (autoensamblado electrostático) Una alternativa para la modificación química de nanocanales es la adsorción electrostática de polielectrolitos. Esta metodología implica sumergir la membrana con el nanocanal en una solución de polielectrolitos con una carga opuesta a la carga presente en las paredes del nanocanal. La modificación con 
polielectrolitos en superficies planas es un proceso muy estudiado[49], más que la adsorción en nanocanales y nanoporos $(R \leq 10 \mathrm{~nm})$, proceso más complejo debido a los efectos del nanoconfinamiento y la difusión que debe realizar el polielectrolito para permear dentro del canal. Para una funcionalización exitosa con el método de adsorción electrostática es necesario considerar cuidadosamente variables tales como: el $\mathrm{pH}$ de la solución, el diámetro del nanocanal, el peso molecular de los polielectrolitos y la fuerza iónica de la solución. Caruso estudio la adsorción de monocapas de polialilaminoa PAH, cargada positivamente, en la superficie interna cargada negativamente de meso-poros de silica[73], así como la adsorción de ácido poliacrilico PAA (cargado negativamente) sobre la superficie positiva de meso-poros de partículas de amino silica. Estos experimentos muestran un decrecimiento en la adsorción cuando se incrementa el peso molecular de los polielectrolitos o una disminución en el tamaño del poro, lo que demuestra limitaciones a la funcionalización dadas por la difusión de polielectrolitos dentro del canal o su capacidad de adsorción en ambientes confinados. Para el PAH, la cantidad adsorbida aumenta cuando aumenta la fuerza iónica, este aumento se atribuye a la formación de ovillos de polielectrolito con el aumento de la concentración de sal[72]. Luego de la adsorción de la primera capa de polielectrolitos, la carga neta en la superficie se invierte y adquiere el mismo signo que la carga del polielectrolito adsorbido, este fenómeno se conoce como inversión de carga[21].

Mediciones de conductancia Para las mediciones de conductancia iónica, se introduce la membrana polimérica con un único nanocanal en una celda de dos compartimentos, construida con material inerte. Cada lado de la celda se llena con la misma solución (típicamente soluciones de $\mathrm{KCl}$, donde ambos iones $\mathrm{K}^{+}$y $\mathrm{Cl}^{-}$tienen mobilidades similares) y un electrodo es introducido en cada una de las secciones de la celda. Al aplicar una diferencia de voltaje entre los electrodos una corriente iónica comienza a fluir a través del sistema. Usando electrodos $\mathrm{Ag} / \mathrm{AgCl}$ la corriente iónica es transformada en una corriente eléctrica según la reacción redox

$$
\mathrm{AgCl}+e^{-} \rightleftharpoons \mathrm{Ag}(s)+\mathrm{Cl}^{-}(a q)
$$

En esta reacción, el cloruro de plata $\mathrm{AgCl}$ es consumido en un electrodo y producido en el otro. La corriente eléctrica es medida mientras circula de un electrodo al otro a través de un circuito externo. Las corrientes en un sistema de nanocanal único son del orden de los nanoamper $\left(10^{-9} A\right)$ y suelen medirse con amplificadores patch clamp[61], como los que se utilizan en electrofisiologia, o con pico/femtoamperímetros[40]. Los sistemas monoporos requieren ser aislados de la radiación electromagnética mediante una jaula de Faraday para reducir el ruido en la señal.

Las mediciones de conductancia son útiles para determinar las propiedades de transporte de nanocanales construidos en las membranas poliméricas. Estas mediciones son la base para muchas aplicaciones analíticas, entre ellas resaltan los experimentos de traslocación de cadenas de ADN[22]. Estos experimentos permiten estudiar rectificación del transporte, aunque no proveen información directa sobre la selectividad iónica (transporte selectivo de aniones o cationes).

\subsection{Interacciones Moleculares}

Para entender el transporte iónico en nanocanales, es útil entender las longitudes características presentes en estos sistemas. Las longitudes características dependen de los cambios en las fuerzas intermoleculares en las cercanías de una superficie sólida. Estos cambios pueden atribuirse a interacciones hidratación (las que se encuentran en un rango de entre 1 y 2 nanó- 


\begin{tabular}{|c|c|}
\hline$C_{\text {salt }}[M]$ & $\lambda_{D}[\mathrm{~nm}]$ \\
\hline \hline $10^{-4}$ & 30,4 \\
\hline $10^{-3}$ & 9,6 \\
\hline $10^{-2}$ & 3,04 \\
\hline $10^{-1}$ & 0,96 \\
\hline 0,150 & 0,78 \\
\hline 1,0 & 0,304 \\
\hline
\end{tabular}

Figura 1.2.1: Longitud de Debye $\left(\lambda_{D}\right)$ en nanómetros para distintos valores de la concentración de sal en una solución acuosa a $25^{\circ} \mathrm{C}$.[45]

metros), interacciones de Van der Waals $(\sim 1-50 \mathrm{~nm})$, o interacciones electrostáticas (longitud de Debye $\left.\lambda_{D} \sim 1-100 \mathrm{~nm}\right)$.

Las interacciones de hidratación se basan en los enlaces de hidrógeno que se forman entre las moléculas de agua, así como entre otras moléculas. Para acercar dos superficies hidrofílicas es necesario romper la red de puentes hidrógeno existente entre ellas. En general, estas interacciones son repulsivas, por estar asociadas a un aumento de la entalpía necesaria para romper los enlaces. Sin embargo, Israelachvili y Pasley midieron las fuerzas de corto alcance entre dos superficies curvas de mica de radio $1 \mathrm{~cm}$ en una solución acuosa $10^{-3} \mathrm{M}$ de $\mathrm{KCl}$ y encontraron que, mientras la fuerza de hidratación es en general repulsiva cuando la distancia entre las superficies está por debajo de los $4 \mathrm{~mm}$, no siempre es monotónica, debajo de los 1, $5 \mathrm{~nm}$ se observa una oscilación cuyo período medio es $0,25 \pm 0,03 \mathrm{~nm}$, muy cercano al diámetro de una molécula de agua. Este experimento muestra que las fuerzas de hidratación pueden ser reguladas.

Las interacciones de Van der Waals ocurren debido a los dipolos inducidos por las fluctuaciones instantáneas de la distribución de cargas alrededor de átomos y moléculas. Típicamente estas interacciones tienen una longitud característica de $\sim 1-50 \mathrm{~nm}$.

Las interacciones electrostáticas ocurren en soluciones iónicas y en las proximidades de superficies cargadas. Para caracterizar la interacción ion-ion, se define la longitud de Bjerrum $\lambda_{B}=\frac{(Z e)^{2}}{4 \pi \varepsilon k_{B} T}$, la distancia entre iones para la cual se alcanza el equilibrio entre la energía electrostática de la interacción ion-ion y la energía térmica. Para iones de valencia $Z=1$ en una solución acuosa a temperatura ambiente $\lambda_{B} \sim 0,7 \mathrm{~nm}$. Por definición, para longitudes menores a $\lambda_{B}$ gobiernan las interacciones electrostáticas por sobre los efectos térmicos.

Una solución de electrolitos en las proximidades de una superficie cargada forma una región donde la carga superficial es neutralizada por una nube de contraiones. Esta región se denomina capa electrostática difusa (EDL, por sus siglas en inglés). Allí la concentración de iones es distinta a la concentración en el seno de la solución debido a la atracción de los contra-iones y la repulsión de los co-iones. En este contexto, a partir de la teoría de Poisson-Boltzmann emerge de forma natural la longitud de Debye $\left(\lambda_{D}\right)$ que caracteriza el apantallamiento electrostático. La longitud de Debye está definida en función de la concentración de sal de la solución, de acuerdo con la expresión: $\lambda_{D}=\sqrt{\left(\varepsilon \varepsilon_{0} k_{B} T / 2 n_{b u l k}(z e)^{2}\right)}$, donde $\varepsilon$ es la constante dieléctrica del agua, $\varepsilon_{0}$ es la permitividad del vacío, $k_{B}$ la constante de Boltzmann, $T$ la temperatura absoluta, $n_{\text {bulk }}$ las concentración de iones en el seno de la solución, $z$ es la valencia del ión y e la carga del electrón. Los valores de $\lambda_{D}$ oscilan entre las decenas de nanómetros hasta unos $3 \AA$, ver tabla 1.2.1.

Las dimensiones de los nanocanales que se pueden construir en la actualidad están en el rango de las longitudes características de cada interacción. Si las soluciones acuosas están confinadas en el rango $1-100 \mathrm{~nm}$, entonces podrían observarse cambios de comportamiento y podrían 


\section{Modeling of Nanopores and Nanochannels}

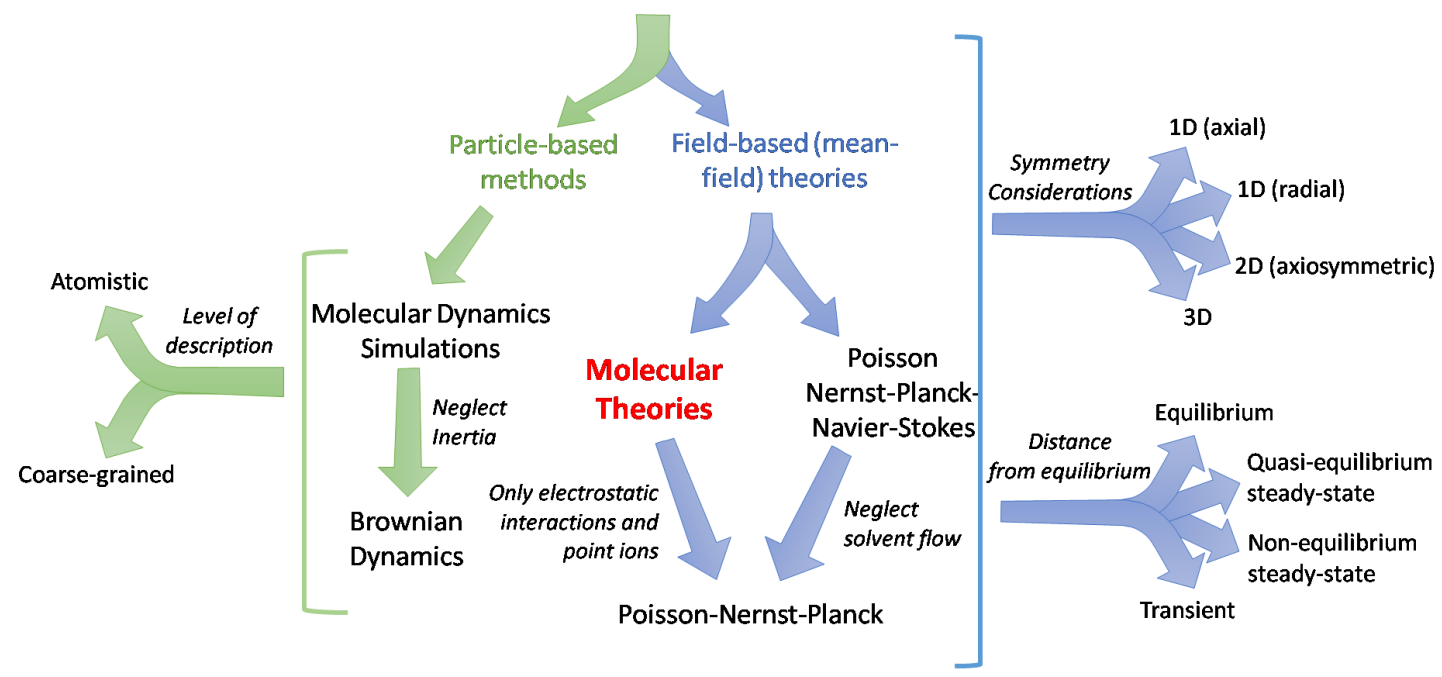

Figura 1.3.1: Mapa de los distintos modelos utilizados para el estudio de nanoporos y nanocanales. La aproximación teórica utilizada en esta tesis corresponde al grupo de las teorías moleculares. Cortesía de M. Tagliazucchi.

desarrollarse estrategias para la manipulación de las propiedades de transporte iónico, haciendo uso de la química de polímeros con el objetivo de realizar aplicaciones ingenieriles.

\subsection{Teoría en nanocanales}

Desde el punto de vista teórico/computacional, se reportan distintas teorías para abordar el transporte en nanocanales. Consideremos los diferentes modelos que pueden usarse para describir el transporte en nanoporos y nanocanales (ver figura 1.3.1). Por un lado, tenemos las teorías basadas en partículas, como por ejemplo las simulaciones de dinámica molecular y dinámica Browniana, que pueden describir sistemas de forma atomística (todos los átomos del sistema son considerados explícitamente) o mediante modelos de grano grueso (conjuntos de átomos se describen agrupados en partículas de grano grueso). Las simulaciones de dinámica molecular atomística son la herramienta más detallada para estudiar el transporte de iones en nanoporos. Esta aproximación ha sido aplicada a nanoporos biológicos y nanocanales. Sin embargo, las longitudes y los tiempos de interés en nanoporos y nanocanales de estado sólido modificados químicamente son más grandes que aquellos que pueden estudiarse con las simulaciones de dinámica molecular atomística. Por ejemplo, el poro biológico típico, $\alpha$-hemolisina, tiene un radio de $\sim 1,3 \mathrm{~nm}$ y una longitud de $10 \mathrm{~nm}$. Está lleno con una solución de electrolitos $1 M$; la $\alpha$-hemolisina contiene $\sim 60$ iones de sal y $\sim 700$ moléculas de agua. Por otro lado un nanoporo con un radio de $6 \mathrm{~nm}$ y una longitud de $50 \mathrm{~nm}$ lleno con una solución $1 \mathrm{M}$ de $\mathrm{KCl}$ contiene $\sim 2 \times 10^{5}$ moléculas de agua y $7 \times 10^{4}$ iones provenientes de la sal (y la situación empeora para nanocanales más largos ). Las simulaciones atomísticas de un sistema con tantas partículas consumen mucho tiempo, incluso disponiendo de supercomputadoras. Alternativamente, los nanocanales de estado sólido pueden describirse en el límite del continuo utilizando métodos de campo medio. Por ejemplo, las ecuaciones de Poisson-Boltzmann (PB) para sistemas en equilibrio y las ecuaciones de Poisson-Nernst-Planck y Navier-Stokes (PNP-NS) para condiciones de no equilibrio. Estas aproximaciones en el continuo han mostrado ser válidas en canales con radios mayores a 2 longitudes de Debye[16, 17] y son relativamente sencillas de 
resolver en una variedad de condiciones. Sin embargo, estos abordajes modelan los iones como partículas puntuales con carga y no incorporan ningún detalle molecular, por lo que no pueden describir el comportamiento de polielectrolitos o proteínas dentro de los nanocanales modificados. Con el objetivo de analizar sistemas moleculares específicos y capturar los acoplamientos existentes entre la organización molecular, las interacciones físicas y los estados químicos, se ha desarrollado una teoría que provee una descripción continua de los campos de interacción, así como de las distribuciones de densidad de las distintas especies, y explícitamente considera el tamaño, forma, distribución de cargas, estados químicos y conformaciones de todas las especies moleculares en el sistema. Esta teoría molecular permite estudiar los nanocanales de estado sólido modificados químicamente porque incluye el detalle químico de las moléculas en el sistema, puede lidiar con las escalas temporales y espaciales apropiadas, y puede ser derivada en un marco teórico formal, lo que permite generalizar la teoría a otros casos de interés. Esta teoría molecular se reduce a las ecuaciones de PNP cuando se consideran únicamente las interacciones electrostáticas y no hay moléculas con grados de libertad conformacionales[65].

Los fenómenos de transporte a través de nanocanales modificados no se limitan al flujo de iones, existen estudios donde se investiga el transporte de estructuras más complejas, como por ejemplo moléculas de ADN, proteínas, o liposomas[29]. Se pueden utilizar estímulos físicos (luz [82, 75, 83], temperatura, diferencias de voltaje[60], etc), químicos (concentración de sal, $\mathrm{pH}$ ) o biológicos (presencia de bioreceptores específicos[74, 6]), para controlar el transporte a través de nanocanales. Desde el punto de vista del comportamiento bioquímico, la capacidad de regular las propiedades de transporte de los nanocanales a través de estímulos externos es importante para el desarrollo de tecnología.

Resulta interesante el paradigma en el cual se intenta enmarcar los futuros avances en el control del transporte iónico. Así como la electrónica ha desarrollado estrategias de control del flujo de electrones, primero con bloques simples como capacitores, resistencias o inductancias, luego con bloques más sofisticados como son los diodos o los transistores hasta llegar a la integración de estos bloques en estructuras mucho más complejas, como por ejemplo circuitos integrados, chips y microchips, así se espera evolucionar en el control de corrientes de iones. Donde el objetivo no es reemplazar la electrónica, sino mas bien integrar de forma natural sensores químicos basados en nanocanales. Es por eso que en los últimos años se ha estado forjando dentro de la comunidad científica el concepto de: iontrónica[15, 51, 38].

\subsection{Sobre este Trabajo}

Este trabajo se realizó en las instalaciones del Instituto Nacional de Investigaciones Fisicoquímicas Teóricas y Aplicas (INIFTA) en el Laboratorio de Materia Blanda ${ }^{1}$ a cargo de Omar Azzaroni, es un trabajo teórico/computacional desarrollado en un grupo eminentemente experimental. Los recursos computacionales utilizados fueron provistos por el grupo de Modelado Molecular de Biomateriales ${ }^{2}$ a cargo del Dr. Szleifer, en donde F. M. Gilles realizó dos estadías.

El contraste entre las herramientas de trabajo, metodología y lenguaje que suelen utilizarse en el laboratorio y las utilizadas en este trabajo, es un ejemplo de la heterogeneidad con la que se trabaja en el grupo en particular y en el ámbito de la Materia Blanda en general. Sea en el laboratorio con equipamiento o en la computadora con líneas de código propio o software específico, en ambos espacios se estudian sistemas complejos que deben abarcarse de forma interdisciplinaria.

\footnotetext{
${ }^{1}$ https://softmatter.quimica.unlp.edu.ar/

${ }^{2}$ http://szleifer-group.bme.northwestern.edu/
} 
Objeto de estudio En este trabajo estudiamos las propiedades de capas poliméricas confinadas dentro de un nanocanal que atraviesa una membrana de polietilentereftalato (PET) de lado a lada. En la figura 2.0.1, se representa gráficamente el orificio que goza de simetría cilíndrica, es decir, sus propiedades geométricas son invariantes ante una rotación respecto de su eje principal. Los diámetros característicos están en el rango de las decenas de nanómetros 1-100 nm. En los sistemas que abordamos la relación de aspecto entre la longitud del canal $L$ y los diámetros característicos $D$, es tal que $L \gg D$. Bajo estas condiciones estos orificios se denominan en la literatura nanocanales de estado sólido. El ejemplo experimental de referencia son aquellos nanocanales que se construyen mediante irradiación iónica sobre películas de PET (polietilenetereftalato). Estas membranas tienen un grosor de $12 \mu \mathrm{m}$ y el orificio que se le practica tiene un radio que depende de los procesos químicos realizados durante la construcción[7]. Típicamente se logran aberturas cuyos radios están en la decena o centena de nanómetros[79, 78, 77, 80, 52].

$\mathrm{El}$ interés en las propiedades de polielectrolitos confinados surge de la necesidad de controlar el transporte iónico a través de nanocanales modificados químicamente. En el laboratorio es posible realizar tratamientos químicos para modificar la geometría e incorporar capas de polielectrolitos en la superficie interna del nanocanal. Para medir el impacto de la modificación superficial sobre el transporte iónico, se realizan experimentos que consisten en medir la corriente iónica $(I)$ en función del potencial externo aplicado $(V)$. Estas corrientes iónicas constituyen la respuesta macroscópica del sistema aunque dependen también de las propiedades geométricas y químicas del nanocanal. Son estas corrientes las que se intentan controlar mediante la funcionalización de la pared interna del nanocanal.

Objetivos del trabajo Es de interés contar con una herramienta teórico/computacional para explicar la respuesta macroscópica en función de las características moleculares involucradas y predecir resultados contrastables. Los objetivos planteados en el plan de trabajo fueron: desarrollar la Teoría Molecular para la descripción de la organización molecular y propiedades de las macromoléculas con las que se modifican los nanocanales de estado sólido (capítulo 2), descripción teórica de la conductancia iónica de los nanocanales modificados con "polymer brushes" (capítulo 3), relacionar las predicciones teóricas y los observables macroscópicos experimentales a fin de interpretar el rol de las diferentes variables del sistema: a) diámetro de poro, b) fuerza iónica, c) pH, d) peso molecular o grado de polimerización de los "polymer brushes" (capítulos 4 y 5$)$. 


\section{Capítulo 2}

\section{Enfoque Teórico}

La teoría molecular que se describe a continuación fue desarrollada originalmente para tratar el autoensamblado de surfactantes[11], más tarde generalizada para estudiar capas de polímeros[13] y más recientemente se incorporó el tratamiento de capas de polielectrolitos[46]. Este enfoque teórico combina un fructífero concepto de la física, como es el hecho de que la naturaleza puede ser descripta a través de una funcional que se debe optimizar[41], una aproximación de campo medio para el tratamiento de las probabilidades de cadenas poliméricas[10] y estrategias de cálculo numérico para la resolución de ecuaciones diferenciales[33].

El objetivo es obtener las propiedades macroscópicas del sistema en estado de equilibrio, junto con la organización molecular que da origen a esas propiedades macroscópicas. Para ello escribimos la energía libre del sistema teniendo en cuenta todas las interacciones relevantes, incluyendo además ciertas restricciones que surgen de la física del problema. Ejemplos de estas restricciones son las condiciones de incomprensibilidad y electroneutralidad global. La energía libre sometida a las restricciones constituye un funcional que llamaremos $\omega$. La minimización del funcional $\omega$ nos permite hallar las distribuciones de todas las especies, la distribución de cargas y el potencial electrostático en cada punto del sistema, en función de las variables accesibles experimentalmente: $p H, C_{\text {salt }}, C_{p o l}$ (concentración de polímero). [48, 47, 46, 44]

Para escribir la teoría se utiliza un sistema de coordenadas con el eje $\hat{z}$ sobre el eje principal del nanocanal y los ejes $\hat{r}$ y $\hat{\theta}$ en el plano perpendicular definen las direcciones radial y angular en coordenadas cilíndricas. El origen de coordenadas se ubica en el centro de la abertura más pequeña del canal que llamaremos "punta" del nanocanal. Para consultar los símbolos y valores utilizados en este trabajo, ver las tablas del apéndice A.

Vamos a considerar nanocanales modificados con polielectrolitos débiles por eso este capítulo comienza con una introducción al equilibrio químico iónico. Estos equilibrios están presentes en los monómeros de polielectrolitos débiles en la forma de un equilibrio ácido-base y tienen su contribución a la energía libre del sistema. Luego se explica en detalle la teoría molecular desarrollada para describir los nanocanales modificados químicamente.

\subsection{Equilibrio Químico}

Consideremos un compuesto cuyos estados neutro $(A H)$ y cargado $\left(A^{-}\right)$están relacionados a través de una reacción química en donde para pasar del estado $A H$ al estado $A^{-}$el compuesto debe liberar un protón $H^{+}$. De forma muy general podemos escribir la reacción química

$$
A H \rightleftharpoons H^{+}+A^{-}
$$




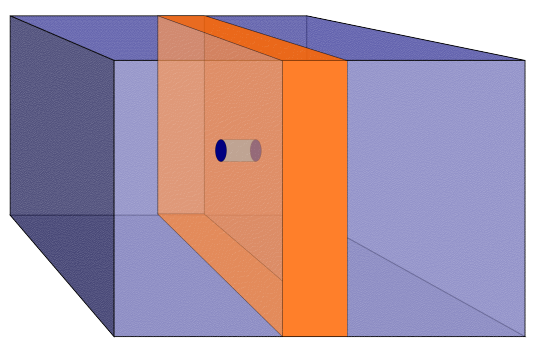

(a) Membrana de PET con un único nanocanal pasante.

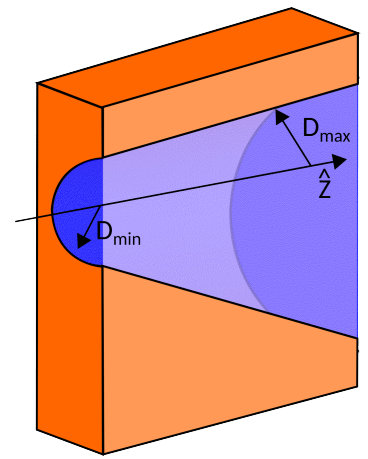

(b) Detalle del nanocanal. Se muestra una geometría asimétrica.

Figura 2.0.1: Esquema del dispositivo experimental y detalle del nanocanal con simetría azimutal. La dirección $\hat{z}$ se elige sobre el eje principal del nanocanal de forma tal que $z=0$ indica el orificio con el diámetro menor. La longitud del nanocanal es mucho más grande que cualquiera de los diámetros presentes en el canal, $(L \gg D)$. Típicamente $L \approx 12 \mu m, D \approx 10-100 \mathrm{~nm}$.

Donde las especies que se indican a la izquierda se denominan reactivos y las especies de la derecha se denominan productos. Esta reacción es un ejemplo de reacción simple ácido-base, donde se dice que $A H$ es un ácido (por que tiene la habilidad de liberar un protón) y $A^{-}$es su base conjugada (por que tiene la posibilidad de capturar un protón). La condición de equilibrio de la reacción química 2.1 implica una relación de equilibrio entre los potenciales químicos de las especies involucradas

$$
\mu_{A H}=\mu_{H^{+}}+\mu_{A^{-}}
$$

donde $\mu_{i}$ representa el potencial químico de la especie $i=A H, A^{-}, H^{+}$. El potencial químico $\mu_{i}$ puede entenderse como la energía libre por partícula de la especie $i$. Es una propiedad útil para describir sistemas inhomogéneos dado que, en la condición de equilibrio, $\mu_{i}$ debe tener el mismo valor en todos los lugares donde puedan acceder las partículas de la especie $i$. Para la descripción del equilibrio químico 2.1 nos alcanza con considerar el potencial químico en su forma más simple,

$$
\mu_{i}=\mu^{0}{ }_{i}+k_{B} T \ln \left(a_{i}\right)
$$

donde $\mu^{0}{ }_{i}$ es el potencial químico standard, $a_{i}$ representa la actividad de cada especie, $k_{B}$ es la constante de Boltzmann y $T$ la temperatura. Con la definición 2.3 el potencial químico tiene unidades de energía por partícula $(J)$, si en lugar de $k_{B} T$ se utiliza la constante de los gases $R=N_{A} k_{B}$, el potencial químico queda en unidades de energía por mol $(\mathrm{J} / \mathrm{mol})$ de partículas. La actividad es una propiedad intensiva adimensional que depende de la elección del estado normal (estado de referencia)[42], en general se dice que el término $k_{B} T \ln \left(a_{i}\right)$ representa la entropía asociada a la especie $i$.

Cada equilibrio iónico/químico tiene asociado una constante de equilibrio $(K)$. Esta constante caracteriza el balance entre las especies involucradas una vez que la reacción alcanzó el equilibrio. Si utilizamos la definición 2.3 en la condición de equilibrio 2.2 , se obtiene

$$
\mu^{0}{ }_{A H}+k_{B} T \ln \left(a_{A H}\right)=\mu^{0}{ }_{A^{-}}+k_{B} T \ln \left(a_{A^{-}}\right)+\mu_{H^{+}}^{0}+k_{B} T \ln \left(a_{H^{+}}\right)
$$




$$
-k_{B} T \ln \left(\frac{a_{A^{-}} a_{H^{+}}}{a_{A H}}\right)=\left(\mu_{A^{-}}^{0}+\mu_{H^{+}}^{0}-\mu_{A H}^{0}\right)
$$

El término de la derecha es una constante que depende únicamente de la elección de los estados de referencia, representa el cambio en la energía libre de Gibbs debido a la reacción, $\Delta G^{0}=\left(\mu_{A H}^{0}-\mu^{0}{ }_{A^{-}}-\mu^{0}{ }_{H^{+}}\right)[32]$. La constante de disociación ácido-base se define como

$$
-\ln \left(K_{t e o}\right)=\frac{\Delta G^{0}}{k_{B} T}=\frac{\left(\mu^{0}{ }_{A^{-}}+\mu^{0} H_{H^{+}}-\mu^{0}{ }_{A H}\right)}{k_{B} T}
$$

y permite calcular la fracción de la actividad de los productos sobre la actividad de los reactivos del equilibrio,

$$
K_{t e o}=\frac{a_{A^{-}} a_{H^{+}}}{a_{A H}}
$$

Típicamente tanto las actividades de los reactivos como la de los productos se aproximan con la concentración molar de las especies. Escribiremos la concentración molar como el símbolo que representa a la especie encerrado entre corchetes, por ejemplo, la concentración molar de la especie A se escribe $[A]$. Bajo esta aproximación la constante de equilibrio que caracteriza la reacción 2.1, se escribe como la fracción de las concentraciones en equilibrio de los productos sobre los reactivos,

$$
K_{\text {exp }}=\frac{[A][H]}{[A H]}
$$

esta expresión tiene la ventaja de ser accesible experimentalmente ya que comprende las concentraciones molares $\left(\mathrm{mol} / \mathrm{dm}^{3}\right)$ de las especies involucradas. De hecho las definiciones teóricas y experimentales de estas cantidades están relacionadas mediante un factor multiplicativo (como se explica en el apéndice B, ecuación B.12).

Es de utilidad definir la fracción de disociación como el cociente entre la concentración de monómeros en un estado determinado sobre el número total de monómeros. Siguiendo con el ejemplo,

$$
\begin{gathered}
f_{A H}=\frac{[A H]}{\left[A^{-}\right]+[A H]} \\
f_{A}=\frac{\left[A^{-}\right]}{\left[A^{-}\right]+[A H]}
\end{gathered}
$$

estas magnitudes miden el grado de disociación. De forma general múltiples equilibrios químicos presentes en una unidad monomérica pueden escribirse como:

$$
\begin{gathered}
A H_{m} \rightleftharpoons A H_{m-1}^{-}+H^{+}, \quad K_{1}=\frac{a_{A H^{-}-a_{H}+}}{a_{A H_{2}}} \\
A H_{m-1}^{-} \rightleftharpoons A H_{m-2}^{2-}+H^{+}, \quad K_{2}=\frac{a_{A H_{n-2}^{2-}} a_{H+}}{a_{A H_{n-1}^{-}}} \\
A H_{m-n+1}^{(n-1)-} \rightleftharpoons A H_{m-n}^{n-}+H^{+}, \quad K_{n}=\frac{a_{A H_{m-n}^{n-} a_{H+}}}{a_{A H_{m-n+1}^{(n-1)-}}} \quad n=1, . ., m
\end{gathered}
$$

donde $K_{n}$ son constantes de equilibrio medibles experimentalmente y están definidas de acuerdo con las actividades de las especies involucradas en el equilibrio químico. $m$ es el número de constantes de equilibrio y $m+1$ el número de estados del monómero. Por definición, 


$$
-\ln \left(K_{n}\right)=\frac{\Delta G_{n}^{0}}{k_{B} T}=\frac{\left(\mu_{A H_{m-n}^{n-}}^{0}+\mu^{0}{ }_{H^{+}}-\mu^{0}{ }_{A H_{m-n+1}^{(n-1)-}}\right)}{k_{B} T}
$$

Utilizando propiedades de recurrencia puede hallarse la siguiente expresión,

$$
\sum_{n=1}^{j} \ln \left(K_{n}\right)=\beta\left(\mu_{A H_{m}}^{0}-\mu^{0}{ }_{A H_{m-j}^{j-j}}-\left(n_{H^{+}, 0}-n_{H^{+}, j}\right) \mu_{H^{+}}^{0}\right)
$$

donde $\beta=1 / k_{B} T$ y $n_{H^{+}, j}$ representa el número de protones en el estado $j\left(A H_{m-j}^{j-}, j=0\right.$ representa el estado $A H_{m}$ ). Despejando la diferencias de potenciales químicos y reemplazando $\mu^{0}{ }_{A H_{m}}$ por $\mu_{0}$, y en general $\mu^{0}{ }_{A H_{m-j}^{j-}}$ por $\mu_{e}$, obtenemos,

$$
\beta\left(\mu_{0}^{0}-\mu_{e}^{0}\right)=\sum_{n=1}^{e} \ln \left(K_{n}\right)+\left(n_{H^{+}, 0}-n_{H^{+}, e}\right) \mu_{H^{+}}^{0}
$$

con $e \geq 1$.

\subsection{Teoría Molecular}

Nuestro objetivo es construir una expresión matemática para la energía libre del nanocanal modificado con cadenas de polielectrolitos. Las propiedades del sistema en estado de equilibrio se podrán obtener mediante la optimización de la energía libre. Por definición podemos escribir la energía libre de Helmholtz según la ecuación $A=-T S+U$. Donde el término $T S$ representa todas las contribuciones entrópicas que consideramos:

- Entropía traslacional de las especies libres $T S_{m i x}$

- Entropía conformacional de las cadenas poliméricas $T S_{p o l}$

- Entropía química más energía química (energía intrínseca), debido a la presencia de equilibrios ácido-base en los segmentos de las cadenas poliméricas $F_{\text {chem }}=-T S_{\text {chem }}+\mu^{0}$, $\left(T S_{\text {chem }}\right)$

y $U$ representa todas las contribuciones energéticas, en nuestro sistema consideramos,

- Interacción entre dos segmentos de cadenas poliméricas $\left(U_{\text {inter }}\right)$

- Interacciones electrostáticas $\left(U_{\text {elec }}\right)$

- Interacción entre la pared del nanocanal y los segmentos de las cadenas poliméricas $\left(U_{\text {wall }}\right)$

Todas las contribuciones energéticas y entrópicas dependen de la organización molecular del sistema y por lo tanto hemos de considerarlas como funciones de la posición dentro del nanocanal. Podemos escribir la energía libre del sistema $A$ en términos de las contribuciones locales, según la expresión 2.10.

$$
A=-T S+U=-T S_{p o l}+\int\left[-T S_{m i x}(\boldsymbol{r})+F_{\text {chem }}(\boldsymbol{r})+U_{\text {wall }}(\boldsymbol{r})+U_{\text {elec }}(\boldsymbol{r})+U_{\text {inter }}(\boldsymbol{r})\right] d \boldsymbol{r}
$$

en donde $\boldsymbol{r}$ representa el vector posición y la integral va sobre todo el nanocanal. 


\subsubsection{Contribuciones Entrópicas}

\section{Entropía de mezcla de especies libres}

El primer termino de la energía libre considera la entropía de mezcla o entropía traslacional de las partículas libres. Si consideramos como partículas libres los iones provenientes de la sal $\mathrm{Cl}^{-}, \mathrm{K}^{+}$, los iones de la solución acuosa $\mathrm{H}^{+}, \mathrm{OH}^{-}$y las moléculas de solvente $w$, la entropía de mezcla se escribe,

$$
\begin{aligned}
-T S_{m i x}(\boldsymbol{r}) & =k_{B} T \rho_{C l^{-}}(\boldsymbol{r})\left[\ln \left(\rho_{C l^{-}}(\boldsymbol{r}) v_{w}\right)-1\right]+k_{B} T \rho_{K^{+}}(\boldsymbol{r})\left[\ln \left(\rho_{K^{+}}(\boldsymbol{r}) v_{w}\right)-1\right] \\
& +k_{B} T \rho_{O H^{-}}(\boldsymbol{r})\left[\ln \left(\rho_{O H^{-}}(\boldsymbol{r}) v_{w}\right)-1\right]+k_{B} T \rho_{H^{+}}(\boldsymbol{r})\left[\ln \left(\rho_{H^{+}}(\boldsymbol{r}) v_{w}\right)-1\right] \\
& +k_{B} T \rho_{w}(\boldsymbol{r})\left[\ln \left(\rho_{w}(\boldsymbol{r}) v_{w}\right)-1\right]
\end{aligned}
$$

donde $v_{w}$ es el volumen de las moléculas de solvente y $\rho_{i}(\boldsymbol{r})$ es la densidad de partículas por unidad de volumen con $i=\mathrm{Cl}^{-}, \mathrm{K}^{+}, w, \mathrm{H}^{+}, \mathrm{OH}^{-}$.

En el capítulo 5 consideramos un nanocanal modificado con un cepillo polimérico, en ese caso no hay contribución de las cadenas del polímero a la entropía de mezcla. En el capítulo 4 en cambio, se consideran cadenas libres y por lo tanto se debe considerar el término de entropía traslacional debida a las cadenas poliméricas. En esta introducción teórica nos ocuparemos de cadenas ancladas a la superficie interna del nanocanal. Los detalles sobre la descripción teórica de cadenas libres se desarrollaron en el capítulo 4.

\section{Entropía configuracional del polímero}

El segundo término en la expresión (2.10) está referido a la entropía configuracional del polímero, luego de realizar la aproximación de campo medio cuyos detalles formales se encuentran en el apéndice E. En el caso de brushes poliméricos las cadenas están pegadas por un extremo a la pared del nanocanal, la entropía configuracional se escribe,

$$
-T S_{p o l}=k_{B} T N_{p} \sum_{\alpha} P(\alpha) \ln (P(\alpha))
$$

donde $N_{P}$ es el número de cadenas pegadas sobre la pared del nanocanal y $P(\alpha)$ la densidad de probabilidad de la conformación $\alpha$.

\section{Equilibrio químico}

La entidad de los polielectrolitos que estemos considerando, estará dada por el equilibrio químico presente en cada una de las unidades monoméricas. Estos equilibrios químicos tienen dos contribuciones a la energía libre del sistema, una contribución entrópica caracterizada por la multiplicidad de estados accesibles a cada monómero y otra contribución debida al potencial químico estándar del estado en que se encuentra el monómero. El tercer término en la energía (2.10) representa la contribución del polielectrolito que estemos considerando. De forma general podemos escribir la energía química como:

$$
F_{\text {chem }}(\boldsymbol{r})=k_{B} T\left\langle\rho_{P}(\boldsymbol{r})\right\rangle\left\{\sum_{e=0}^{m} f_{e}(\boldsymbol{r})\left[\ln \left(f_{e}(\boldsymbol{r})\right)+\beta \mu_{e}^{0}\right]\right\}
$$


donde $\left\langle\rho_{p}(\boldsymbol{r})\right\rangle$ es la densidad de monómeros en la posición $\boldsymbol{r}, \mu_{e}^{0}$ es el potencial químico standard del estado $e$. Salvo que se aclare, utilizamos números enteros, con el símbolo $e$, para nombrar los estados químicos de una unidad monomérica. Como asumimos equilibrio químico local escribimos la fracción de disociación dependiente de las coordenadas espaciales $\boldsymbol{r}: f_{e}(\boldsymbol{r})$. Dado que los equilibrios ácido-base incrementan en una unidad la carga eléctrica neta de la especie, el número de estado $e$, identifica (salvo a lo sumo el signo) la carga neta de la especie. Por ejemplo, en el caso del PMEP(figura 2.2.3), si le asignamos $e=0$ al estado de carga nula, la fracción de monómeros en el estado con carga nula será: $f_{0}$, la fracción de monómeros en el estado de carga -1 será $f_{1}$ y en general, la fracción de monómeros con carga neta $q=-e$ será $f_{e}$. En este contexto $e$ es sólo un entero.

Por definición la suma de todas las fracciones de disociación debe sumar uno, es decir:

$$
\sum_{e=0}^{m} f_{e}(\boldsymbol{r})=1
$$

Esta condición permite reducir el número de funciones $f_{e}(\boldsymbol{r})$ independientes. Elegimos escribir el grado de disociación del estado $e=0$ como dependiente de las fracciones de monómeros de los estados cargados: $f_{0}(\boldsymbol{r})=1-\sum_{e \neq 0} f_{e}(\boldsymbol{r})$.

Teniendo en cuenta la expresión para la fracción de monómeros con carga nula: $f_{0}(\boldsymbol{r})=$ $1-\sum_{e=1}^{m} f_{e}(\boldsymbol{r})$ puede re-escribirse la expresión para la energía química en términos de las constantes de equilibrio

$$
F_{\text {chem }}(\boldsymbol{r})=k_{B} T\left\langle\rho_{P}(\boldsymbol{r})\right\rangle\left\{\sum_{e=1}^{m} f_{e}(\boldsymbol{r})\left[\ln \left(\frac{f_{e}(\boldsymbol{r})}{f_{0}(\boldsymbol{r})}\right)+\beta\left(\mu_{e}^{0}-\mu_{0}^{0}\right)\right]+\ln \left(f_{0}(\boldsymbol{r})\right)+\beta \mu_{0}^{0}\right\}
$$

ahora usamos 2.8 reescrita en términos del número de estado $e$ junto con la ecuación 2.9: $\sum_{n=1}^{e} \ln \left(K_{n}\right)+\left(n_{H^{+}, 0}-n_{H^{+}, e}\right) \mu_{H^{+}}^{0}=\beta\left(\mu_{0}^{0}-\mu_{e}^{0}\right)$.

$$
\begin{array}{r}
F_{\text {chem }}(\boldsymbol{r})=k_{B} T\left\langle\rho_{P}(\boldsymbol{r})\right\rangle\left\{\sum_{e=1}^{m} f_{e}(\boldsymbol{r})\left[\ln \left(\frac{f_{e}(\boldsymbol{r})}{f_{0}(\boldsymbol{r})}\right)-\sum_{n=1}^{e} \ln \left(K_{n}\right)-\left(n_{H^{+}, 0}-n_{H^{+}, e}\right) \mu_{H^{+}}^{0}\right]\right. \\
\left.+\ln \left(f_{0}(\boldsymbol{r})\right)+\beta \mu_{0}^{0}\right\}
\end{array}
$$

En el contexto de la teoría molecular es posible incorporar la arquitectura de las cadenas de polielectrolitos, ver figura 2.2.1. Las variaciones en la arquitectura se incorporan a través de la densidad de monómeros $\left\langle\rho_{p}(\boldsymbol{r})\right\rangle$. El procedimiento consiste en escribir las contribuciones de los distintos tipos de monómeros a la energía libre:

$$
\begin{aligned}
F_{\text {chem }}(\boldsymbol{r}) & =k_{B} T \sum_{\zeta}\left\langle\rho_{P}(\boldsymbol{r})\right\rangle_{\zeta}\left\{\sum_{e=1}^{m} f_{e}(\boldsymbol{r})\left[\ln \left(\frac{f_{e}(\boldsymbol{r})}{f_{0}(\boldsymbol{r})}\right)-\sum_{n=1}^{e} \ln \left(K_{n}\right)-\left(n_{H^{+}, 0}-n_{H^{+}, e}\right) \mu_{H^{+}}^{0}\right]\right. \\
& \left.+\ln \left(f_{0}(\boldsymbol{r})\right)+\beta \mu_{0}^{0}\right\}_{\zeta}
\end{aligned}
$$

donde $\zeta$ corre sobre los tipos de monómeros que se consideren y la densidad de monómeros local total es: $\left\langle\rho_{p}(\boldsymbol{r})\right\rangle=\sum_{\zeta} \rho_{p \_\zeta}(\boldsymbol{r})$. En este trabajo se consideran solo arquitecturas homogéneas como las primeras tres de la figura 2.2.1.

Si se consideran equilibrios ácido base que acoplan con la especie $\mathrm{OH}^{-}$en lugar del protón 


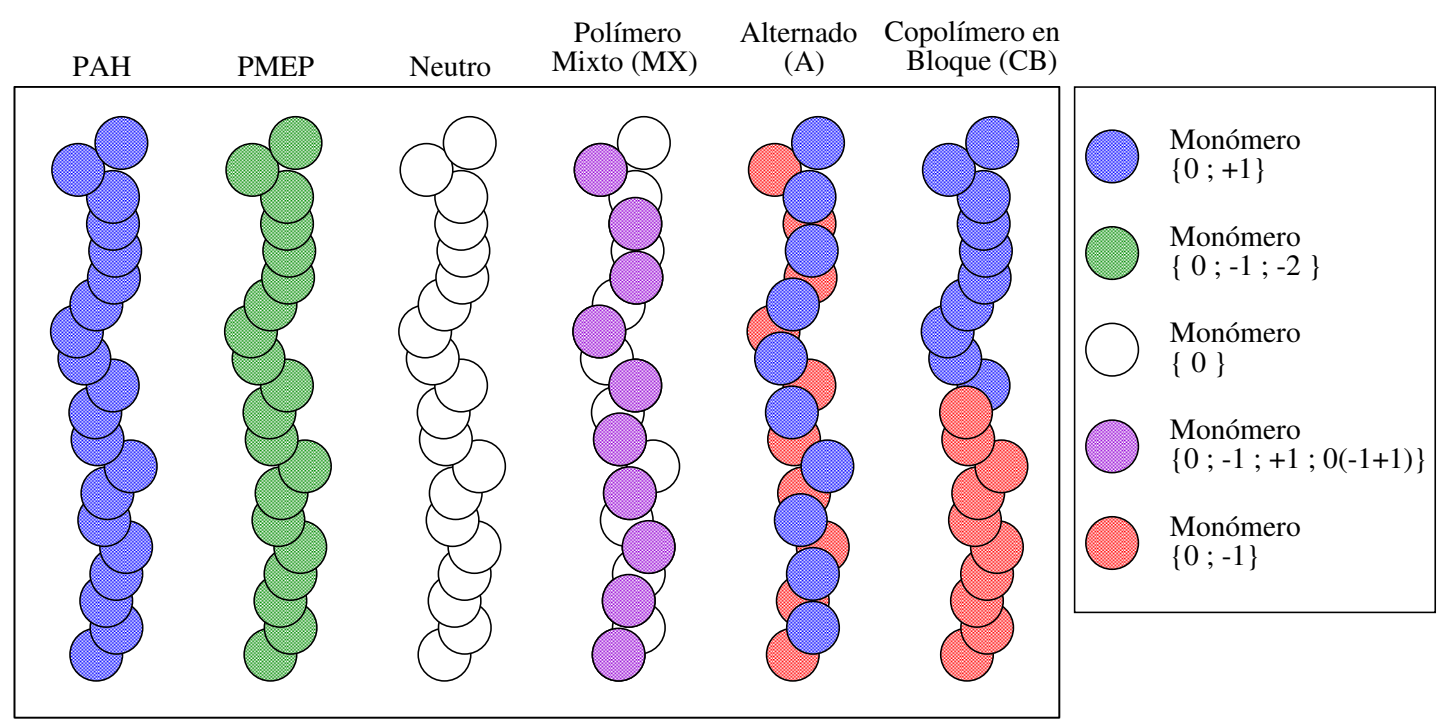

Figura 2.2.1: Arquitectura para las cadenas de polielectrolitos, susceptibles de ser incorporadas a la teoría molecular mediante el cálculo de la densidad de monómeros local de cada especie de monómero. En este trabajo consideramos sólo las primeras dos arquitecturas de cadenas homogéneas para las especies de PMEP y PAH.

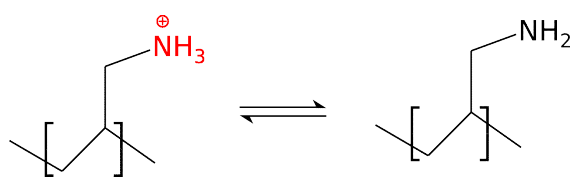

Figura 2.2.2: Estructura y equilibrio ácido-base del monómero de polialilamina (PAH)

$H^{+}$, la deducción anterior es análoga. A continuación se escriben explícitamente las expresiones 2.13 y 2.15 para dos casos particulares de polielectrolitos: polialilamina (PAH) y poli(2(metacriloiloxi) etil-fosfato (PMEP).

Primer Caso Polialilamina $(\boldsymbol{P} \boldsymbol{A} \boldsymbol{H}): \quad$ El PAH es un polielectrolito con dos estados de carga. El estado neutro con carga nula y el estado 1 con carga $+1 e$ (donde $e=-1,602 \times 10^{-19} C$ es la carga fundamental). Considérese ahora el equilibrio químico presente en las unidades del polímero de PAH. La reacción de bulk se escribe cómo,

$$
\mathrm{H}_{2} \mathrm{O}+\mathrm{B \rightleftharpoons} \mathrm{BH}^{+}+\mathrm{OH}^{-}, \quad K_{b}=\frac{a_{B H^{+}} a_{O H^{-}}}{a_{B}}
$$

este equilibrio químico acopla con la actividad de la especie $O H^{-}$(más diferencias con el acoplamiento del $H^{+}$se harán evidentes cuando escribamos las restricciones del entorno). Siguiendo la analogía con los pasos anteriores se obtiene

$$
\beta \Delta G^{0}=\beta\left(\mu_{1}^{0}+\mu_{O H^{-}}^{0}-\mu_{0}^{0}\right)=-\ln \left(K_{b}\right)
$$

donde el estado 0 corresponde a la especie $B$ y el estado 1 a la especie $B H^{+}$. La energía asociada con este equilibrio se escribe como,

$$
F_{\text {chem }}(\boldsymbol{r})=k_{B} T\left\langle\rho_{p}(\boldsymbol{r})\right\rangle\left\{f_{0}(\boldsymbol{r})\left[\ln \left(f_{0}(\boldsymbol{r})\right)+\beta \mu_{B}^{0}\right]+f_{1}(\boldsymbol{r})\left[\ln \left(f_{1}(\boldsymbol{r})\right)+\beta \mu_{B H^{+}}\right]\right\}
$$

mientras que la contribución a la energía libre en términos de la constante de equilibrio $K_{b}$ 


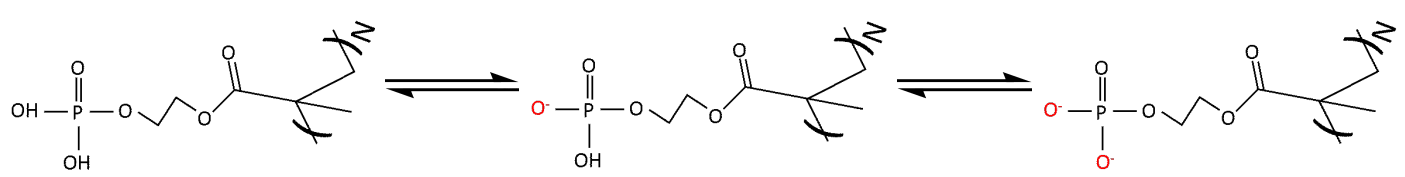

Figura 2.2.3: Estructura y equilibrio ácido-base del monómero de poli(2-(metacriloiloxi) etilfosfato (PMEP)

queda

$$
F_{\text {chem }}(\boldsymbol{r})=k_{B} T\left\langle\rho_{P}(\boldsymbol{r})\right\rangle\left\{f_{1}(\boldsymbol{r})\left[\ln \left(\frac{f_{1}(\boldsymbol{r})}{f_{0}(\boldsymbol{r})}\right)-\ln \left(K_{b}\right)-\beta \mu_{H^{+}}^{0}\right]+\ln \left(f_{0}(\boldsymbol{r})\right)+\beta \mu_{0}^{0}\right\}
$$

El valor de la constante de equilibrio para la amina en los monómeros de PAH $p K_{b}=3,4$ se corresponde con el $p K_{b}$ de una amina primaria[43] .

Segundo Caso Poli(2-(metacriloiloxi) etilfosfato (PMEP): En el caso del monómero poliprótico 2-(methacriloiloxi) etil fosfato $(P M E P)$ reconocemos que tiene tres estados de carga: totalmente protonado $\left(M E P H_{2}\right.$, estado 0$)$ con carga neta nula, parcialmente protonado $\left(M E P H^{-}\right.$, estado 1$)$ con carga neta $q=-1$ y no protonado $\left(M E P^{2-}\right.$, estado 2$)$ con carga neta $q=-2$. Este monómero tiene dos equilibrios químicos asociados caracterizados por las constantes de equilibrio $K_{a 1}=10^{-4,5}$ y $K_{a 2}=10^{-7,7}$. Estos equilibrios se corresponden con el caso $m=2$ en la ecuación general 2.7. La energía química del PMEP en la energía libre (2.10) se escribe cómo,

$$
\begin{aligned}
& F_{\text {chem }}(\boldsymbol{r})=k_{B} T\left\langle\rho_{p}(\boldsymbol{r})\right\rangle\left\{f_{0}(\boldsymbol{r})\left[\ln \left(f_{0}(\boldsymbol{r})\right)+\beta \mu_{A H_{2}}^{0}\right]+\right. \\
&\left.f_{-1}(\boldsymbol{r})\left[\ln \left(f_{-1}(\boldsymbol{r})\right)+\beta \mu_{A H^{-}}\right]+f_{-2}(\boldsymbol{r})\left[\ln \left(f_{-2}(\boldsymbol{r})\right)+\beta \mu_{A^{--}}^{0}\right]\right\}
\end{aligned}
$$

la contribución a la energía libre en términos de las constantes de equilibrio $K_{a 1}$ y $K_{a 2}$ queda,

$$
\begin{array}{r}
F_{\text {chem }}(\boldsymbol{r})=k_{B} T\left\langle\rho_{P}(\boldsymbol{r})\right\rangle\left\{\sum_{e=1}^{2} f_{e}(\boldsymbol{r})\left[\ln \left(\frac{f_{e}(\boldsymbol{r})}{f_{0}(\boldsymbol{r})}\right)-\sum_{n=1}^{e} \ln \left(K_{n}\right)-\left(n_{H^{+}, 0}-n_{H^{+}, e}\right) \mu_{H^{+}}^{0}\right]\right. \\
\left.+\ln \left(f_{0}(\boldsymbol{r})\right)+\beta \mu_{0}^{0}\right\}
\end{array}
$$

de forma explícita,

$$
\begin{aligned}
F_{\text {chem }}(\boldsymbol{r}) & =k_{B} T\left\langle\rho_{P}(\boldsymbol{r})\right\rangle\left\{f_{1}(\boldsymbol{r})\left[\ln \left(\frac{f_{1}(\boldsymbol{r})}{f_{0}(\boldsymbol{r})}\right)-\ln \left(K_{a 1}\right)-\beta \mu_{H^{+}}^{0}\right]\right. \\
& \left.+f_{2}(\boldsymbol{r})\left[\ln \left(\frac{f_{2}(\boldsymbol{r})}{f_{0}(\boldsymbol{r})}\right)-\ln \left(K_{a 1} K_{a 2}\right)-2 \beta \mu_{H^{+}}^{0}\right]+\ln \left(f_{0}(\boldsymbol{r})\right)+\beta \mu_{0}^{0}\right\}
\end{aligned}
$$

La densidad promedio $\left\langle\rho_{P}(\boldsymbol{r})\right\rangle$ representa la densidad de monómeros del polielectrolito por unidad de volumen. Su expresión en términos de la densidad de probabilidad de las confor- 
maciones de cadena depende del sistema que estemos considerando. La expresión (2.19) es la densidad de monómeros en el caso de un brush polimérico, en dónde $N_{p}$ representa el número de polímeros pegados en la superficie, $A(R)$ es el área de la superficie interna del nanoporo, $n(\boldsymbol{r} ; \alpha)$ es el número de monómeros en $\boldsymbol{r}$ pertenecientes a la conformación $\alpha$, y $P(\alpha)$ es la probabilidad de la conformación $\alpha$.

$$
\left\langle\rho_{P}(\boldsymbol{r})\right\rangle=\frac{N_{P}}{A(R)} \sum_{\alpha} n_{p}(\boldsymbol{r}, \alpha) P(\alpha)
$$

La expresión (2.19), es el resultado de la aproximación de campo medio donde asumimos que la probabilidad de hallar una cadena polimérica en la conformación $\alpha$ es independiente de otras conformaciones de cadena. (detalles de la aproximación se encuentran en el apéndice E).

\subsubsection{Interacciones Intermoleculares}

\section{Energía de interacción Pared-Polímero}

Independientemente del estado de carga de los monómeros del polímero podemos modelar las interacciones de Van der Waals entre la pared y los monómeros del polielectrolito a través de un potencial de la forma

$$
U_{\text {wall }}(\boldsymbol{r})=-\chi_{\text {wall }}(\boldsymbol{r})\left\langle\rho_{P}(\boldsymbol{r})\right\rangle
$$

en el caso de cadenas de polielectrolitos adsorvidas sobre la pared del nanocanal, utilizamos un parámetro: $\chi_{\text {wall }}(\boldsymbol{r})=\left\{\begin{array}{ll}\epsilon & r>R-\delta \\ 0 & r<R-\delta\end{array}\right.$ de interacción pared-monómero, donde $r$ es el módulo del vector posición $\boldsymbol{r}$

\section{Energía Electrostática}

La quinta contribución que consideramos en (2.10) corresponde a la energía electrostática, la expresión microscópica se escribe como,

$$
U_{\text {elect }}(\boldsymbol{r})=\left[\left\langle\rho_{q}(\boldsymbol{r})\right\rangle \Psi(\boldsymbol{r})-\frac{1}{2} \varepsilon\left(\nabla_{r} \Psi(\boldsymbol{r})\right)^{2}\right]
$$

donde $\Psi(\boldsymbol{r})$ es el potencial electrostático, $\varepsilon$ es la permitividad del solvente y $\left\langle\rho_{q}(\boldsymbol{r})\right\rangle$ es la densidad de carga media. Esta magnitud considera la carga de las especies libres: $\mathrm{H}^{+}, \mathrm{OH}^{-}$, los iones provenientes de la sal $\mathrm{Cl}^{-}, \mathrm{K}^{+}$y la carga proveniente de los monómeros del polímero. La densidad de carga promedio se escribe considerando los estados cargados de las unidades monoméricas, por ejemplo para el caso del MEP, existen tres estados $(e=0,1,2)$ cada uno con carga $q_{e}=-e$, entonces:

$$
\left\langle\rho_{q}(\boldsymbol{r})\right\rangle=-\left(f_{1}(\boldsymbol{r})+2 f_{2}(\boldsymbol{r})\right)\left\langle\rho_{p}(\boldsymbol{r})\right\rangle+\sum_{i=K^{+}, C l^{-}, H^{+}, O H^{-}} q_{i} \rho_{i}(\boldsymbol{r})
$$

Más en general si un segmento tiene $m+1$ estados entonces $e=0, \ldots, m$ y cada estado tiene una carga $q_{e}$, la densidad de carga promedio se escribe como, 


$$
\begin{gathered}
\left\langle\rho_{q}(\boldsymbol{r})\right\rangle=\left(\sum_{e=0}^{m} f_{e}(\boldsymbol{r}) q_{e}\right)\left\langle\rho_{p}(\boldsymbol{r})\right\rangle+q_{H^{+}} \rho_{H^{+}}(\boldsymbol{r})+q_{O H^{-}} \rho_{O H^{-}}(\boldsymbol{r}) \\
+q_{K^{+}} \rho_{K^{+}}(\boldsymbol{r})+q_{C l^{-}} \rho_{C l^{-}}(\boldsymbol{r})
\end{gathered}
$$

\section{Interacción de corto alcance - Van der Waals atractivo}

Las interacciones de Van de Waals están representadas por un término cuadrático en la energía libre, tienen en cuenta la calidad del solvente y la distribución no homogénea de los monómeros del polímero. La energía asociada a esta interacción cadena-cadena, $U_{\text {inter }}(r)$, está relacionada con las variables microscópicas a través de la expresión, (2.23),

$$
U_{\text {inter }}(\boldsymbol{r})=\frac{1}{2} k_{B} T \int d \boldsymbol{r}^{\prime} \chi\left(\boldsymbol{r}-\boldsymbol{r}^{\prime}\right)\left\langle\phi_{p}(\boldsymbol{r})\right\rangle\left\langle\phi_{p}\left(\boldsymbol{r}^{\prime}\right)\right\rangle
$$

donde $k_{B}$ es la constante de Boltzmann, $T$ la temperatura, $\left\langle\phi_{p}(\boldsymbol{r})\right\rangle$ es la fracción en volumen de polímero en la posición $\boldsymbol{r}$ y finalmente $\chi\left(\boldsymbol{r}-\boldsymbol{r}^{\prime}\right)$ es un parámetro que caracteriza la interacción de Van der Waal entre los segmentos de polímero ubicados en las posiciones $\boldsymbol{r}$ y $\boldsymbol{r}^{\prime}$. En este trabajo vamos a considerar únicamente la condición de buen solvente $\chi\left(\boldsymbol{r}-\boldsymbol{r}^{\prime}\right)=0$ para todo $\boldsymbol{r}-\boldsymbol{r}^{\prime}$. Esto significa que los monómeros del polímero no tienen preferencia entre otros monómeros y moléculas del solvente.

\subsubsection{Intercambio de Partículas con el Entorno: Ensamble Gran Ca- nónico}

Todos estos términos constituyen la energía libre del sistema donde el número de partículas es constante. Sin embargo el sistema se encuentra en equilibrio termodinámico con un entorno cuyas propiedades están bien definidas: $p H$ de la solución, concentración de sal $C_{\text {salt }}$, concentración de polímero $C_{p o l}$, temperatura $T$. Se hace necesario realizar las transformaciones de Legendre para usar un ensamble gran canónico donde el número de iones dentro del nanocanal es variable pero el número de cadenas poliméricas es constante. La energía libre en el nuevo ensamble queda escrita como

$$
\begin{aligned}
& A=\int\left[-T S_{\text {mix }}(\boldsymbol{r})-T S_{\text {pol }}(\boldsymbol{r})+F_{\text {chem }}(\boldsymbol{r})+U_{\text {wall }}(\boldsymbol{r})+U_{\text {elec }}(\boldsymbol{r})+U_{\text {inter }}(\boldsymbol{r})\right] d \boldsymbol{r} \\
& -\int\left[\mu_{w} \rho_{w}(\boldsymbol{r})+\mu_{C l^{-}} \rho_{C l^{-}}(\boldsymbol{r})+\mu_{K^{+}} \rho_{K^{+}}(\boldsymbol{r})+\mu_{O H^{-}}\left(\rho_{O H^{-}}(\boldsymbol{r})+\sum_{e} n_{e, O H^{-}} f_{e}(\boldsymbol{r})\left\langle\rho_{p}(\boldsymbol{r})\right\rangle\right)\right. \\
& \left.\quad+\mu_{H^{+}}\left(\rho_{H^{+}}(\boldsymbol{r})+\sum_{e} n_{e, H^{+}} f_{e}(\boldsymbol{r})\left\langle\rho_{p}(\boldsymbol{r})\right\rangle\right)\right] d \boldsymbol{r}
\end{aligned}
$$

donde $n_{e, O H^{-}}$y $n_{e, H^{+}}$son el número de iones de hidroxilo $\left(\mathrm{OH}^{-}\right)$o hidronio $\left(\mathrm{H}^{+}\right)$presentes en el estado $e$. Naturalmente esto depende del tipo de equilibrios químicos presentes en el sistema. La expresión 2.24 considera equilibrios químicos cuyo acoplamiento ocurre con la concentración de $\mathrm{OH}^{-}$y equilibrios químicos que acoplan con las concentraciones de $H^{+}$. Ver la tabla 2.1 para una descripción de los estados presentes en PMEP y en PAH. Por ejemplo, en el caso del PAH, el número total de hidronios $H^{+}$es el número de hidronios libres $H^{+}$y el número de iones hidroxilos $O H^{-}$es $n_{0, O H^{-}}=0$ en el estado $e=0$ y $n_{1, O H^{-}}=1$, en el estado $e=1$. 


\begin{tabular}{|c|c|c|c|c|c|}
\hline \multicolumn{6}{|c|}{$A H_{n-1}^{-} \rightleftharpoons A H_{n-2}^{2-}+H^{+}, \quad K_{-2}=\frac{\left[A H_{n-2}^{2-}\right]\left[H^{+}\right]}{\left[A H_{n-1}^{-}\right]}$} \\
\hline \multicolumn{6}{|c|}{$B \rightleftharpoons B H^{+}+O H^{-}, \quad K_{1}=\frac{\left[B H^{+}\right]\left[O H^{-}\right]}{[B]}$} \\
\hline & Símbolo & $e$ & $n_{e, H^{+}}$ & $n_{e, O H^{-}}$ & Observaciones \\
\hline \multirow{2}{*}{$\mathrm{PAH}$} & $B$ & $\overline{0} 0$ & $\overline{0}$ & 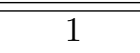 & \multirow[b]{2}{*}{$n_{e, O H^{-}}=1-e$} \\
\hline & $B H^{+}$ & 1 & 0 & 0 & \\
\hline \multirow{3}{*}{ PMEP } & $\mathrm{AH}_{2}$ & 0 & 2 & 0 & \multirow{3}{*}{$n_{e, H^{+}}=2+e$} \\
\hline & $A H^{-}$ & -1 & 1 & 0 & \\
\hline & $A^{2-}$ & -2 & 0 & 0 & \\
\hline
\end{tabular}

Cuadro 2.1: Tabla de valores y notación para los equilibrios ácido-base presentes en los monómeros de PAH y PMEP. Estos valores aparecen en la expresión 2.28.

Es de utilidad dejar la expresión 2.24 expresada en términos de los grados de disociación independientes,

$$
\begin{gathered}
A=\int\left[-T S_{m i x}(\boldsymbol{r})-T S_{p o l}(\boldsymbol{r})+F_{c h e m}(\boldsymbol{r})+U_{\text {wall }}(\boldsymbol{r})+U_{\text {elec }}(\boldsymbol{r})+U_{\text {inter }}(\boldsymbol{r})\right] d \boldsymbol{r} \\
-\int\left[\mu_{w} \rho_{w}(\boldsymbol{r})+\mu_{C l^{-}} \rho_{C l^{-}}(\boldsymbol{r})+\mu_{K^{+}} \rho_{K^{+}}(\boldsymbol{r})\right. \\
+\mu_{O H^{-}}\left(\rho_{O H^{-}}(\boldsymbol{r})+\left[\sum_{e=1}^{m}\left(n_{e, O H^{-}}-n_{0, O H^{-}}\right) f_{e}(\boldsymbol{r})+n_{0, O H^{-}}\right]\left\langle\rho_{p}(\boldsymbol{r})\right\rangle\right) \\
\left.+\mu_{H^{+}}\left(\rho_{H^{+}}(\boldsymbol{r})+\left[\sum_{e=1}^{m}\left(n_{e, H^{+}}-n_{0, H^{+}}\right) f_{e}(\boldsymbol{r})+n_{0, H^{+}}\right]\left\langle\rho_{p}(\boldsymbol{r})\right\rangle\right)\right] d \boldsymbol{r}
\end{gathered}
$$

\subsubsection{Restricciones a la Energía Libre: Empaquetamiento, Electro- neutralidad y Normalización de la Probabilidad de Configura- ciones $P(\alpha)$}

Las interacciones de volumen excluido son incorporadas en la teoría de forma explícita de dos formas. Por un lado las interacciones repulsivas entre segmentos de una misma cadena, interacciones intramoleculares, se incorporan de forma exacta al considerar solo conformaciones que no se superponen con sigo misma (self-avoiding). Por otro lado las interacciones de volumen excluido entre moléculas se incorporan a través de la condición de incomprensibilidad (condición de empaquetamiento), ecuación 2.29 .

$$
\left\langle\phi_{P}(\boldsymbol{r})\right\rangle+\sum_{i} \phi_{i}(\boldsymbol{r})=1 \quad \text { para cada } \boldsymbol{r}
$$

Formalmente debemos incorporar también la normalización de la probabilidad de las conformaciones de cadena $P(\alpha)$. Para ello vamos a considerar la normalización local, es decir vamos a pedir que la probabilidad $P(\alpha)$ cumpla con la ecuación 2.30 .

$$
\sum_{\alpha} P(\alpha)=1
$$




$$
\sigma_{q} f_{\text {wall }}+\int d \boldsymbol{r}\left\langle\rho_{q}(\boldsymbol{r})\right\rangle=0
$$

La condición 2.30 hace que la constante que normaliza la probabilidad no dependa explícitamente del tamaño del sistema. Estas condiciones junto con la condición de electroneutralidad ecuación 2.31, se imponen sobre la energía libre a través de los multiplicadores de Lagrange, $\pi(\boldsymbol{r}), Z, \mathrm{y} \lambda$. Los tres términos que se deben sumar a la expresión de la energía libre 2.24, son

$$
I_{R}=\int d \boldsymbol{r} \beta \pi(\boldsymbol{r})\left[\left\langle\phi_{P}(\boldsymbol{r})\right\rangle+\sum_{i} \phi_{i}(\boldsymbol{r})-1\right]+\lambda\left[\sigma_{q}+\int d \boldsymbol{r} \beta\left\langle\rho_{q}(\boldsymbol{r})\right\rangle\right]+Z\left(\sum_{\alpha} P(\alpha)-1\right)
$$

Habiendo presentado todos los términos relevantes para la descripción del sistema, a continuación vamos a restringir la energía libre de nuestro sistema a las condiciones $\left(I_{R}\right)$.

Cuando el sistema se encuentra en equilibrio termodinámico con su entorno, sus variables de estado deben optimizar la funcional $\omega=\frac{\beta}{\pi D L}\left(A+I_{R}\right)$, donde $L$ es el largo y $D$ el diámetro del nanocanal. Esta funcional $\omega$ está definida como la energía libre del sistema en el ensamble gran canónico más las restricciones 2.32 y representa la energía total por unidad de área y energía térmica $k_{B} T$, de la superficie modificada. En la expresión (2.33) se muestra la expresión completa de la funcional escrita en coordenadas cilíndricas. 


$$
\begin{aligned}
& \omega=\int_{0}^{R} \rho_{C l^{-}}(r)\left[\ln \left(\rho_{C l^{-}}(r) v_{w}\right)-1\right]\left(\frac{r}{R}\right) d r+\int_{0}^{R} \rho_{K^{+}}(\boldsymbol{r})\left[\ln \left(\rho_{K^{+}}(\boldsymbol{r}) v_{w}\right)-1\right]\left(\frac{r}{R}\right) d r \\
& +\int_{0}^{R} \rho_{O H^{-}}(r)\left[\ln \left(\rho_{O H^{-}}(r) v_{w}\right)-1\right]\left(\frac{r}{R}\right) d r+\int_{0}^{R} \rho_{H^{+}}(r)\left[\ln \left(\rho_{H^{+}}(r) v_{w}\right)-1\right]\left(\frac{r}{R}\right) d r \\
& +\int_{0}^{R} \rho_{w}(r)\left[\ln \left(\rho_{w}(r) v_{w}\right)-1\right]\left(\frac{r}{R}\right) d r+\int_{0}^{R} \rho_{P C}(r)\left[\ln \left(\rho_{P C}(r) v_{w}\right)-1\right]\left(\frac{r}{R}\right) d r \\
& +\sigma \sum_{\alpha} P(\alpha) \ln (P(\alpha)) \\
& +\int_{0}^{R}\left\langle\rho_{P}(r)\right\rangle\left\{\sum _ { e = 1 } ^ { m } f _ { e } ( r ) \left[\ln \left(\frac{f_{e}(r)}{f_{0}(r)}\right)-\sum_{n=1}^{e} \ln \left(K_{n}\right)-\left(n_{H^{+}, 0}-n_{H^{+}, e}\right) \beta \mu_{H^{+}}^{0}\right.\right. \\
& \left.\left.-\left(n_{O H^{-}, 0}-n_{O H^{-}, e}\right) \beta \mu_{O H^{-}}^{0}\right]+\ln \left(f_{0}(r)\right)+\beta \mu_{0}^{0}\right\}\left(\frac{r}{R}\right) d r \\
& -\int_{0}^{R} \beta \chi_{\text {wall }}(r)\left\langle\rho_{P}(r)\right\rangle\left(\frac{r}{R}\right) d r+\int_{0}^{R} \beta\left[\left\langle\rho_{q}(r)\right\rangle \Psi(r)-\frac{1}{2} \varepsilon\left(\nabla_{r} \Psi(r)\right)^{2}\right]\left(\frac{r}{R}\right) d r \\
& +\frac{1}{2} \int_{0}^{R} \int_{0}^{R} d r^{\prime}\left(2 \pi r^{\prime}\right) \chi\left(r, r^{\prime}\right)\left\langle\phi_{p}(r)\right\rangle\left\langle\phi_{p}\left(r^{\prime}\right)\right\rangle\left(\frac{r}{R}\right) d r \\
& -\int_{0}^{R} \beta\left[\mu_{w} \rho_{w}(r)+\mu_{C l^{-}} \rho_{C l^{-}}(r)+\mu_{K^{+}} \rho_{K^{+}}(r)\right. \\
& +\mu_{O H^{-}}\left(\rho_{O H^{-}}(r)+\left[\sum_{e=1}^{m}\left(n_{e, O H^{-}}-n_{0, O H^{-}}\right) f_{e}(r)+n_{0, O H^{-}}\right]\left\langle\rho_{p}(r)\right\rangle\right) \\
& \left.+\mu_{H^{+}}\left(\rho_{H^{+}}(r)+\left[\sum_{e=1}^{m}\left(n_{e, H^{+}}-n_{0, H^{+}}\right) f_{e}(r)+n_{0, H^{+}}\right]\left\langle\rho_{p}(r)\right\rangle\right)\right]\left(\frac{r}{R}\right) d r \\
& +\int_{0}^{R} \beta \pi(r)\left[\left\langle\phi_{P}(r)\right\rangle+\sum_{i} \phi_{i}(r)-1\right]\left(\frac{r}{R}\right) d r+\int_{0}^{R} \beta \lambda\left[\sigma_{q} f_{\text {wall }}+\int d r \beta\left\langle\rho_{q}(r)\right\rangle\right]\left(\frac{r}{R}\right) d r \\
& +Z\left(\sum_{\alpha} P(\alpha)-1\right)
\end{aligned}
$$

El siguiente paso es reorganizar los términos para la posterior minimización analítica de la funcional. El multiplicador de Lagrange $\lambda$ representa la contribución global al potencial electrostático que asegura electroneutralidad, vemos que puede absorberse dentro del potencial electrostático. Redefinimos el potencial electrostático como $\Psi(r)=\Psi^{*}(r)+\lambda$. Los casos que consideraremos serán todos bajo la condición de buen solvente $\chi\left(\boldsymbol{r}, \boldsymbol{r}^{\prime}\right)=0$. Luego de reorganizar los términos redujimos la expresión al caso de cadenas ancladas a la superficie del nanocanal (ver capítulo 4 para el caso de cadenas libres). Como hemos visto cada término en la expresión 2.33 tiene significado físico, por lo que es posible diferencias entre las distintas contribuciones a la energía. Luego de la reorganización de los términos en la funcional $\omega$, ecuación 2.34, ya no 
es posible identificar las distintas contribuciones.

$$
\begin{gathered}
\omega=\int_{0}^{R} \rho_{C l^{-}}(r)\left[\ln \left(\rho_{C l^{-}}(r) v_{w}\right)-1-\beta \mu_{C l^{-}}+\beta \pi(r) v_{C l^{-}}\right]\left(\frac{r}{R}\right) d r \\
+\int_{0}^{R} \rho_{K^{+}}(r)\left[\ln \left(\rho_{K^{+}}(r) v_{w}\right)-1-\beta \mu_{K^{+}}+\beta \pi(r) v_{K^{+}}\right]\left(\frac{r}{R}\right) d r \\
+\int_{0}^{R} \rho_{O H^{-}}(r)\left[\ln \left(\rho_{O H^{-}}(r) v_{w}\right)-1-\beta \mu_{O H^{-}}+\beta \pi(r) v_{O H^{-}}\right]\left(\frac{r}{R}\right) d r \\
+\int_{0}^{R} \rho_{H^{+}}(r)\left[\ln \left(\rho_{H^{+}}(r) v_{w}\right)-1-\beta \mu_{H^{+}}+\beta \pi(r) v_{H^{+}}\right]\left(\frac{r}{R}\right) d r \\
+\int_{0}^{R} \rho_{w}(r)\left[\ln \left(\rho_{w}(r) v_{w}\right)-1-\beta \mu_{w}+\beta \pi(r) v_{w}\right]\left(\frac{r}{R}\right) d r \\
+\int_{0}^{R}\left\langle\rho_{P}(r)\right\rangle\left\{\sum _ { e = 1 } ^ { m } f _ { e } ( r ) \left[\ln \left(\frac{f_{e}(r)}{f_{0}(r)}\right)-\sum_{n=1}^{e} \ln \left(K_{n}\right)-\left(n_{e, H^{+}}-n_{0, H^{+}}\right) \beta \Delta \mu_{H^{+}}\right.\right. \\
\left.-\left(n_{e, O H^{-}}-n_{0, O H^{-}}\right) \beta \Delta \mu_{o H^{-}}\right]-n_{0, O H^{-}} \beta \mu_{O H^{-}}-n_{0, H^{+}} \beta \mu_{H^{+}} \\
\left.+\ln \left(f_{0}(r)\right)+\beta \mu_{0}^{0}-\beta \chi_{w a l l}(r)+\beta \pi(r) v_{p}\right\}\left(\frac{r}{R}\right) d r \\
\quad+\int_{0}^{R} \beta\left[\left\langle\rho_{q}(r)\right\rangle \Psi(r)-\frac{1}{2} \varepsilon\left(\nabla_{r} \Psi(r)\right)^{2}\right]\left(\frac{r}{R}\right) d r \\
+\sigma \sum_{\alpha} P(\alpha) \ln (P(\alpha))-\int_{0}^{R} \beta \pi(r)\left(\frac{r}{R}\right) d r+\beta Z\left(\sum_{\alpha} P(\alpha)-1\right)
\end{gathered}
$$

Los términos que involucran al potencial químico de las especies $\mathrm{OH}^{-}$y $\mathrm{H}^{+}$y el potencial químico standard del estado $0, \mu_{0}^{0}$ contribuyen en una constante a la funcional por lo tanto pueden omitirse. La expresión completa de la funcional 2.33 y la compacta 2.34 serán de utilidad al momento de realizar el cálculo variacional para encontrar las expresiones del equilibrio. Finalmente, dejamos las expresiones para la densidad de carga local dentro del canal 2.35

$$
\left\langle\rho_{q}(r)\right\rangle=\sum_{i=K^{+}, C l^{-}, O H^{-}, H^{+}} q_{i}\left\langle\rho_{i}(r)\right\rangle+\sum_{e=1}^{m_{e}} q_{e} f_{e}(r)\left\langle\rho_{p}(r)\right\rangle, i=C l^{-}, O H^{-}, K^{+}, H_{3} O^{+}
$$

\subsection{Optimización Analítica y Expresiones Resultantes}

El procedimiento para obtener las propiedades de equilibrio del sistema consiste en optimizar la expresión (2.34) mediante cálculo variacional (ver apéndice F). La aparición de las derivadas del potencial electrostático $(\Psi(r))$ en la expresión de la funcional (2.34) trae como consecuencia natural del cálculo variacional la ecuación de Poisson, una ecuación diferencial para el potencial electrostático que debe ser discretizada (ver apéndice D) y resuelta de forma autoconsistente mediante cálculo numérico. Para el resto de las variables $P(\alpha), \rho_{i}(r), i=\mathrm{Cl}^{-}, \mathrm{OH}^{-}, \mathrm{K}^{+}$, $H^{+}, f_{e}(r)$, se obtienen expresiones en función de los valores de bulk y de los campos (presión osmótica $\pi(r)$ y potencial electrostático $\Psi(r))$.

\section{Optimización con respecto a la densidad numérica $\left(\rho_{i}(r)\right)$, comparación con bulk}

Luego de realizar la minimización con respecto a la densidad volumétrica $\rho_{i}(r)$ con $i=$ $w, \mathrm{Cl}^{-}, \mathrm{OH}^{-}, \mathrm{K}^{+}, \mathrm{H}^{+}$, se obtienen expresiones (2.36), que relacionan las densidades de las 
distintas especies químicas con el potencial electrostático local, la presión osmótica local y el potencial químico de la especie.

$$
\beta \mu_{i}=\ln \left(\phi_{i}(r) \frac{v_{w}}{v_{i}}\right)+\beta v_{i} \pi(r)+\beta q_{i} \Psi(r)
$$

Las expresiones explícitas para las moléculas de solvente y los iones libres, son:

$$
\begin{gathered}
\beta \mu_{w}=\ln \left(\rho_{w}(r) v_{w}\right)+\beta v_{w} \pi(r) \\
\beta \mu_{K^{+}}=\ln \left(\phi_{K^{+}}(r) \frac{v_{w}}{v_{K^{+}}}\right)+\beta v_{K^{+}} \pi(r)+\beta q_{K^{+}} \Psi(r) \\
\beta \mu_{C l^{-}}=\ln \left(\phi_{C l^{-}}(r) \frac{v_{w}}{v_{C l^{-}}}\right)+\beta v_{C l^{-}} \pi(r)+\beta q_{C l^{-}} \Psi(r) \\
\beta \mu_{H^{+}}=\ln \left(\phi_{H^{+}}(r) \frac{v_{w}}{v_{H^{+}}}\right)+\beta v_{H^{+}} \pi(r)+\beta q_{H^{+}} \Psi(r) \\
\beta \mu_{O H^{-}}=\ln \left(\phi_{O H^{-}}(r) \frac{v_{w}}{v_{O H^{-}}}\right)+\beta v_{O H^{-}} \pi(r)+\beta q_{O H^{-}} \Psi(r)
\end{gathered}
$$

Es posible definir los potenciales químicos del resto de las especies como potenciales químicos de intercambio entre moléculas de solvente $w$ y moléculas de la especie $i$, como $\Delta \mu_{i}^{*}=\mu_{i}-$ $\frac{v_{i}}{v_{w}} \mu_{w}[28,46]$, para $i=K^{+}, H^{+}, C l^{-}, O H^{-}$. Así definidas estas cantidades representan la energía necesaria para cambiar una molécula de solvente por un ion. El potencial químico de intercambio de las moléculas de solvente es cero, $\Delta \mu_{w}^{*}=0$, y de la ecuación (2.37), se puede calcular la presión osmótica $\pi(r)$ del sistema como el logaritmo natural de la fracción de volumen del solvente $\phi_{w}(r)=\rho_{w}(r) v_{w}$.

$$
\beta v_{w} \pi(r)=-\ln \left(\rho_{w}(r) v_{w}\right)
$$

La expresión (2.37) permite entender el significado de la presión osmótica como la fuerza que el solvente tiene que hacer para mantener su potencial químico constante en el sistema (condición de equilibrio). Aquellos lugares donde la densidad del solvente decrece la presión osmótica deberá incrementarse. Las expresiones para los potenciales químicos de intercambio quedan:

$$
\beta \mu_{i}^{*}=\ln \left(\phi_{i}(r) \frac{v_{w}}{v_{i}}\right)-\frac{v_{i}}{v_{w}} \ln \left(\rho_{w}(r) v_{w}\right)+\beta q_{i} \Psi(r)
$$

De ahora en adelante los potenciales químicos se escriben como potenciales químicos de intercambio, es decir asumimos la relación 2.42. Las mismas expresiones pueden escribirse para los potenciales químicos en bulk, donde consideramos $\Psi^{\text {bulk }}=0$.

$$
\beta \mu_{i}=\ln \left(\phi_{i}^{b} \frac{v_{w}}{v_{i}}\right)-\frac{v_{i}}{v_{w}} \ln \left(\phi_{w}^{b}\right), i=w, C l^{-}, O H^{-}, K^{+}, H^{+}
$$




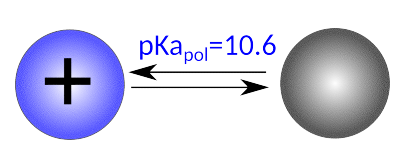

Figura 2.3.1: Equilibrio químico presente en los monómeros de PAH.

Como en (2.37), el lado izquierdo de las ecuaciones (2.38-2.41) es constante debido a la condición de equilibrio. Estas ecuaciones muestran el acoplamiento entre el potencial electrostático, la presión osmótica y la densidad de iones libres. Es útil reescribir las fracciones de volumen de iones y la fracciones de volumen del solvente como función de las propiedades de bulk, el potencial eléctrico y la presión osmótica.

$$
\begin{gathered}
\phi_{w}(r)=\phi_{w}^{b} \exp \left\{-\beta v_{w}\left(\pi(r)-\pi^{b}\right)\right\} \\
\phi_{i}(r)=\frac{v_{i}}{v_{w}} \exp \left\{\beta \mu_{i}\right\} \exp \left\{-\beta v_{i}\left(\pi(r)-\pi^{b}\right)\right\} \exp \left\{-\beta q_{i} \Psi(r)\right\} \\
\phi_{i}(r)=\frac{v_{i}}{v_{w}} \exp \left\{\beta \mu_{i}\right\}\left(\frac{\phi_{w}(r)}{\phi_{w}^{b}}\right)^{v_{i} / v_{w}} \exp \left\{-\beta q_{i} \Psi(r)\right\}
\end{gathered}
$$

Las ecuaciones 2.44 y 2.46 nos permiten calcular las fracciones de volumen de todas las especies químicas dentro del nanocanal una vez conocidos el potencial electrostático $\Psi(r)$, la presión osmótica $\pi(r)$ y las condiciones del bulk.

\section{Expresiones para el grado de disociación: $f_{e}(r)$}

La expresión general que se obtiene para el grado de disociación $f_{e}(r)$ del estado de los monómeros es

$$
\begin{aligned}
\ln \left(\frac{f_{e}(r)}{f_{0}(r)}\right) & =\sum_{n=1}^{e} \ln \left(K_{n}\right)+\left(n_{H^{+}, e}-n_{H^{+}, 0}\right) \beta \Delta \mu_{H^{+}} \\
& +\left(n_{O H^{-}, e}-n_{O H^{-}, 0}\right) \beta \Delta \mu_{O H^{-}}-\beta q_{e} \Psi(r)
\end{aligned}
$$

con $e=1, \ldots, m_{e}$, recordar que $f_{0}(r)=1-\sum_{e=1}^{m_{e}} f_{e}(r)$. La ecuación 2.47 es importante por que es una relación general para cualquier equilibrio químico de la forma (2.5-2.7). A continuación se presentan dos ejemplos de polielectrolitos explorados durante el transcurso de este trabajo.

PAH En el caso del PAH el grado de disociación es una protonación simple, $m_{e}=1$, y la minimización es respecto de un único grado de disociación, $f_{1}(r)$ (recordar que $f_{0}(r)=1-f_{1}(r)$ ) La expresión que se obtiene es,

$$
\begin{gathered}
\ln \left(\frac{f_{1}(r)}{f_{0}(r)}\right)=-\ln \left(K_{b}\right)-\beta \Delta \mu_{O H^{-}}-\beta q_{1} \Psi(r) \\
\ln \left(\frac{f_{1}(r)}{f_{0}(r)}\right)=\ln \left(K_{b} e^{-\beta \Delta \mu_{O H^{-}}} e^{-\beta q_{1} \psi(r)}\right)
\end{gathered}
$$

De donde se puede deducir las expresiones para las fracciones de disociación 


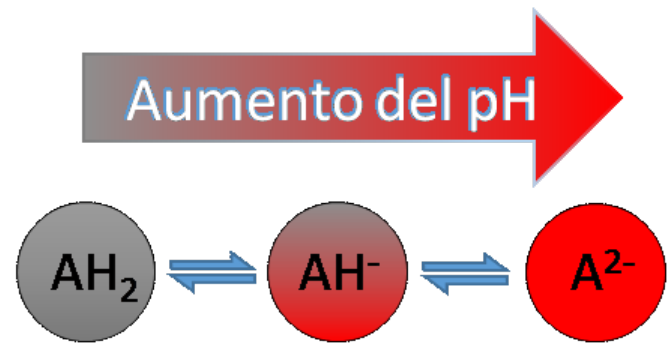

Figura 2.3.2: Transición entre los estados del PMEP en la dirección de aumento del pH de la solución.

$$
\begin{aligned}
& f_{0}(r)=\frac{1}{1+K_{b} e^{-\beta \Delta \mu_{O H^{-}}} e^{-\beta q_{1} \psi(r)}} \\
& f_{1}(r)=f_{0}(r) K_{b} e^{-\beta \Delta \mu_{O H^{-}}} e^{-\beta q_{1} \psi(r)}
\end{aligned}
$$

Para la implementación numérica se utilizó la siguiente expresión,

$$
f_{1}(r)=\frac{1}{1+e^{\beta \Delta \mu_{O H^{-}}} e^{\beta q_{1} \psi(r)} \frac{1}{K_{b}}}=\frac{1}{1+\frac{1}{K_{b}} \rho_{O H^{-}}(r)\left(\frac{\phi_{w}^{b}}{\phi_{w}(r)}\right)}
$$

donde se utilizó que $v_{w}=v_{O H}$. (Ver tabla A.3)

PMEP Para el caso del PMEP (doble deprotonación, $m_{e}=2$ ), y la minimización con respecto a los grados independientes de disociación $f_{e}(r)$ con $e=-1,-2$ (e recorre números negativos haciendo alusión a la carga neta del monómero en ese estado) (recordar que $f_{0}(r)=$ $\left.1-f_{-1}(r)-f_{-2}(r)\right)$ resultan en las expresiones explícitas

$$
\begin{gathered}
\beta \Delta \mu_{H^{+}}=\ln \left(K_{1}\right)-\ln \left(\frac{f_{-1}(r)}{f_{0}(r)}\right)-\beta q_{-1} \Psi(r) \\
2 \beta \Delta \mu_{H^{+}}=\ln \left(K_{1} K_{2}\right)-\ln \left(\frac{f_{-2}(r)}{f_{0}(r)}\right)-\beta q_{-2} \Psi(r) \\
\ln \left(\frac{f_{-1}(r)}{f_{0}(r)}\right)=\ln \left(K_{1} e^{-\beta \Delta \mu_{H^{+}}} e^{-\beta q_{-1} \Psi(r)}\right) \\
\ln \left(\frac{f_{-2}(r)}{f_{0}(r)}\right)=\ln \left(K_{1} K_{2} e^{-2 \beta \Delta \mu_{H^{+}}} e^{-\beta q_{-2} \Psi(r)}\right)
\end{gathered}
$$

Los términos de la derecha en las expresiones 2.50 y 2.51 , muestran que debe existir un balance entre el potencial eléctrico y los grados de disociación debido a la condición de potencial químico de los protones constante. Es posible encontrar una expresión para los grados de disociación, $f_{-1}(r)$ y $f_{-2}(r)$. Si usamos la condición $f_{0}(r)+f_{-1}(r)+f_{-2}(r)=1$, junto con las últimas dos expresiones 2.52 y 2.53 , podemos encontrar las ecuaciones de los grados de disociación como función de las densidades locales del solvente $\phi_{w}(r)$ y el hidrógeno $\phi_{H^{+}}(r)$.

$$
f_{0}(r)=\frac{\left(\frac{\phi_{H^{+}}(r)}{\phi_{w}(r)}\right)^{2}}{\left(\frac{\phi_{H^{+}}(r)}{\phi_{w}(r)}\right)^{2}+\frac{\phi_{H^{+}}(r)}{\phi_{w}(r)} K_{1}+K_{1} K_{2}}
$$




$$
\begin{aligned}
f_{-1}(r) & =\frac{\frac{\phi_{H^{+}}(r)}{\phi_{w}(r)} K_{1}}{\left(\frac{\phi_{H}+(r)}{\phi_{w}(r)}\right)^{2}+\frac{\phi_{H^{+}}(r)}{\phi_{w}(r)} K_{1}+K_{1} K_{2}} \\
f_{-2}(r) & =\frac{K_{1} K_{2}}{\left(\frac{\phi_{H^{+}}(r)}{\phi_{w}(r)}\right)^{2}+\frac{\phi_{H^{+}}(r)}{\phi_{w}(r)} K_{1}+K_{1} K_{2}}
\end{aligned}
$$

Optimización con respecto a la probabilidad de las conformaciones poliméricas: $P(\alpha)$

Para la función probabilidad de conformaciones $P(\alpha)$ de polímero encontramos que,

$$
\begin{aligned}
0=\sigma[\ln ( & P(\alpha))+1]-Z \\
+ & \sigma \int_{0}^{R} n_{p}(r ; \alpha)\left\{\sum_{e=1}^{m} \beta q_{e} f_{e}(r) \Psi(r)+\beta v_{p} \pi(r)+\ln \left(f_{0}(r)\right)-\beta \chi_{\text {wall }}(r)\right. \\
& +\sum_{e=1}^{m} f_{e}(r)\left[\ln \left(\frac{f_{e}(r)}{f_{0}(r)}\right)-\sum_{n=1}^{e} \ln \left(K_{n}\right)-\left(n_{e, H^{+}}-n_{0, H^{+}}\right) \beta \Delta \mu_{H^{+}}\right. \\
& \left.\left.\quad-\left(n_{e, O H^{-}}-n_{0, O H^{-}}\right) \beta \Delta \mu_{o H^{-}}\right]\right\}\left(\frac{r}{R}\right) d r
\end{aligned}
$$

Es posible, verificar que si $Z=\sigma\left(1+\ln \left(\frac{1}{Q}\right)\right)$ entonces $Q=e^{1-Z / \sigma}$ es la función de partición del sistema que normaliza la probabilidad de conformación de una sola cadena $P(\alpha)$. Donde,

$$
\begin{gathered}
Q=\sum_{\alpha} \exp \left(-\int_{0}^{R} n(r ; \alpha) \times\left\{\beta\left(\sum_{e=1}^{m_{e}} q_{e} f_{e}(r)\right) \Psi(r)+\beta \pi(r) v_{p}+\ln \left(f_{0}(r)\right)-\beta \chi_{\text {wall }}(r)\right.\right. \\
+\sum_{e=1}^{m} f_{e}(r)\left[\ln \left(\frac{f_{e}(r)}{f_{0}(r)}\right)-\sum_{n=1}^{e} \ln \left(K_{n}\right)-\left(n_{e, H^{+}}-n_{0, H^{+}}\right) \beta \Delta \mu_{H^{+}}\right. \\
\left.\left.-\left(n_{e, O H^{-}}-n_{0, O H^{-}}\right) \beta \Delta \mu_{o H^{-}}\right]\right\}\left(\frac{r}{R}\right) d r
\end{gathered}
$$

Si sabemos el grado de disociación independiente $\left(f_{e}(r)\right)$, el potencial eléctrico $(\Psi(r))$ y la presión osmótica $(\pi(r))$, es posible calcular la probabilidad de la conformación $\alpha$. En los ejemplos estudiados,

$$
\begin{array}{r}
P(\alpha)=\frac{1}{Q} \exp \left(-\int_{0}^{R} n(r ; \alpha) \times\left\{\beta\left(\sum_{e=1}^{m_{e}} q_{e} f_{e}(r)\right) \Psi(r)+\beta \pi(r) v_{p}+\ln \left(f_{0}(r)\right)-\beta \chi_{\text {wall }}(r)\right.\right. \\
+\sum_{e=1}^{m} f_{e}(r)\left[\ln \left(\frac{f_{e}(r)}{f_{0}(r)}\right)-\sum_{n=1}^{e} \ln \left(K_{n}\right)-\left(n_{e, H^{+}}-n_{0, H^{+}}\right) \beta \Delta \mu_{H^{+}}\right. \\
\left.\left.\left.-\left(n_{e, O H^{-}}-n_{0, O H^{-}}\right) \beta \Delta \mu_{o H^{-}}\right]\right\}\left(\frac{r}{R}\right) d r\right)
\end{array}
$$




\section{Optimización con respecto al potencial electrostático: $\psi(r)$}

La ecuación de Poisson 2.59 se obtiene como resultado del cálculo variacional de la energía libre (2.34) respecto del potencial eléctrico $\Psi(r)$.

$$
\nabla^{2} \psi(r)=-\frac{\left\langle\rho_{q}(r)\right\rangle}{\varepsilon}
$$

La ecuación 2.59 y la expresión 2.58 deben ser discretizadas para ser resueltas computacionalmente. Los detalles del procedimiento se encuentran en el apéndice D.

\subsubsection{Expresión para la Energía Reducida}

Si reemplazamos las expresiones obtenidas luego del cálculo variacional 2.44, 2.46, $2.47 \mathrm{y}$ 2.58, en la expresión de la energía libre 2.33, se obtiene lo que llamamos la energía libre reducida. Se interpreta como la energía del sistema en el punto estacionario, estado de equilibrio del sistema.

$$
\begin{aligned}
w=-\sum_{i=w, K^{+}, H^{+}, O H^{-}, C l^{-}} \int_{0}^{R} \rho_{i}(r)\left(\frac{r}{R}\right) d r+\frac{1}{v_{w}} \int_{0}^{R} & \ln \left(\phi_{w}(r)\right)\left(\frac{r}{R}\right) d r \\
& -\frac{1}{2} \int_{0}^{R} \rho_{q}(r) \beta \Psi(r)\left(\frac{r}{R}\right) d r-\sigma \ln (Q)
\end{aligned}
$$

Las soluciones del sistema, ecuaciones 2.42, 2.46, 2.49(para el PAH), 2.55 y 2.56 (para el PMEP), 2.58 y 2.59, se resuelven de forma auto-consistente. Primero discretizamos las expresiones (ver apéndice D) para luego hallar las soluciones de manera iterativa. El procedimiento consiste en proponer una solución inicial para la fracción de solvente $\left(\phi_{w}^{(0)}(r)=1\right)$ y el potencial electrostático $\left(\Psi^{(0)}(r)=0\right)$. Luego se calculan las distribuciones de partículas con las ecuaciones 2.46 y los grados de disociación. Finalmente se recalculan el potencial electrostático $\Psi^{(1)}(r)$ y la fracción de solvente $\phi_{w}^{(1)}(r)$ a través de la ecuación de Poisson 2.59 y la condición de empaquetamiento 2.29. Con $\Psi^{(1)}(r), \phi_{w}^{(1)}(r)$ se calcula la energía libre del sistema. El procedimiento se detiene cuando la distancia entre las soluciones $\Psi^{(n-1)}(r), \phi_{w}^{(n-1)}(r)$ y $\Psi^{(n)}(r)$, $\phi_{w}^{(n)}(r)$ es menor a un valor de corte, típicamente $10^{-8}$.

Para confirmar que los resultados obtenidos computacionalmente son compatibles se compara el valor numérico de la energía libre reducida con el valor numérico de la expresión general 2.33 . 


\section{Capítulo 3}

\section{Propiedades de Transporte}

La corriente iónica a través de una solución es un evento más complejo que el flujo de electrones a través de un metal. En el metal, los electrones de masa despreciable llevan toda la corriente mientras que en la solución iónica, existe un flujo de iones positivos y negativos, de masa no despreciable. Por consiguiente, el paso de una corriente va acompañado de un transporte de masa. Como los iones positivos y negativos tienen distinta mobilidad no llevan porciones iguales de la corriente total, de modo que se desarrollan gradientes de concentración en la solución. Además, la transferencia de carga a través de la interfaz solución-electrodo se acompaña de una reacción química (electrólisis) en cada electrodo. En este capítulo nos ocuparemos únicamente del fenómeno de transporte en la solución confinada dentro de nanocanales largos.

Si frente a un potencial externo aplicado la estructura del sistema permanece similar a la estructura del sistema en estado de equilibrio entonces es posible calcular la conductancia iónica a partir de las propiedades del equilibrio. La información que provee la teoría molecular resulta útil para estimar la conductancia del sistema en el límite de cuasi-equilibrio, límite en el cuál el potencial externo aplicado es pequeño. En este capítulo mostramos cuál es la conexión entre la organización molecular en el equilibrio y la conductancia de nanocanales largos en el régimen de cuasi-equilibrio, ecuación 3.4. Luego apartir de la conductancia de nanocanales cilíndricos desarrollamos un método para extrapolar estos resultados a geometrías más generales, ecuaciones 3.11, 3.15. Finalmente, mostramos como se calcula el valor medio de propiedades moleculares en geometrías más generales, expresión 3.18 .

\subsection{Conductancia $(G)$ de Nanocanales}

En un sistema con comportamiento óhmico la relación lineal entre el potencial aplicado $V$ y la corriente $I$ puede expresarse en función de la resistencia o la conductancia,

$$
\begin{aligned}
& V=R \times I \\
& I=G \times V
\end{aligned}
$$

Por su definición la conductancia coincide con la inversa de la resistencia. Y la resistividad coincide con la inversa de la conductividad. Expresar la relación entre el potencial $V$ y la corriente iónica $I$, en términos de la conductancia pone de manifiesto la variable experimental controlada $(V)$ que representa el estímulo externo y la variable medida $(I)$ que representa la respuesta del sistema. Los gráficos típicos en la caracterización de estos sistemas corresponden 


\begin{tabular}{|c|c|c|}
\hline $\mathrm{M}\left(\mathrm{mol} / \mathrm{dm}^{3}\right)$ & Conductividad & IUPAC \\
\hline \hline 1 & & $111.3 \frac{\mathrm{mS}}{\mathrm{cm}}$ \\
\hline 0.1 & $12.88 \frac{\mathrm{mS}}{\mathrm{cm}} \pm 1 \%$ & $12.85 \frac{\mathrm{mS}}{\mathrm{cm}}$ \\
\hline 0.01 & $1413 \frac{\mu \mathrm{S}}{\mathrm{cm}} \pm 1 \%$ & $1408 \frac{\mathrm{\mu S}}{\mathrm{cm}}$ \\
\hline 0.001 & $146.9 \frac{\mu \mathrm{S}}{\mathrm{cm}} \pm 2,5 \%$ & \\
\hline
\end{tabular}

Cuadro 3.1: Valores de la conductancia para distintas concentraciones de $\mathrm{KCl}$. Conductividad standard $\mathrm{KCl} 0,1 \mathrm{M}, 12,88 \mathrm{mS} / \mathrm{cm}$

a curvas $I$ vs $V$ (respuesta del sistema en función del estímulo externo).

La diferencia de voltaje entre dos electrodos separados por una membrana con un único nanocanal pasante, genera corrientes iónicas $(I)$ a través del nanocanal. La conductancia $(G)$ de un nanocanal cilíndrico con paredes neutras, se puede obtener conociendo las dimensiones del nanocanal y la conductividad $(\kappa)$ de la solución,

$$
G=\kappa\left(\frac{\pi R^{2}}{L}\right)
$$

la conductividad $(k)$ es la capacidad de un medio para conducir cargas y tiene unidades de $\left[\frac{S}{m}\right]^{1}$. Típicamente la conductividad de una solución depende de la concentración de sal en la solución (ver tabla $3.1,{ }^{2}$ ). Un modelo sencillo para la conductividad de la solución que da cuenta de la dependencia con la concentración de sal $C_{\text {salt }}$ es la ecuación: $\kappa=\left(\mu_{K}+\mu_{C l}\right) C_{\text {salt }} e$, donde $\mu_{K}$ y $\mu_{C l}$ representan las mobilidades de las especies iónicas ${ }^{3}$.

Con el objetivo de estimar la conductancia de canales cilíndricos, utilizamos los resultados de la teoría molecular junto con las ecuaciones de Nernst-Planck bajo la aproximación de campo constante utilizada por Goldman[27]. Como estamos considerando nanocanales largos $(L \gg R)$ la resistencia al transporte de carga iónica estará dada por lo que ocurre dentro del canal y no por lo que ocurre en sus bordes. Estas aproximaciones permiten calcular la conductancia iónica $(G)$ como la suma de la conductividades de cada una de las especies iónica móviles. Las conductividades se calculan utilizando el coeficiente de difusión $D_{i}$ de cada ion $i=\mathrm{K}^{+}, \mathrm{Cl}^{-}, \mathrm{OH}^{-}, \mathrm{H}^{+}$y la concentración promedio dentro del nanocanal $c_{i}(r)$ calculada con la teoría molecular con potencial aplicado nulo (condición de equilibrio). Con el flujo iónico en la dirección $\hat{z}$ y condiciones de equilibrio dentro del poro, la conductividad del nanocanal queda determinada por,

$$
G=\frac{I}{V}=\frac{2 \pi}{L} \frac{F^{2}}{R T} \sum_{i=K^{+}, C l^{-}, O H^{-}, H^{+}} q_{i}{ }^{2} D_{i} \int_{0}^{R} c_{i}(r) r d r
$$

donde $D_{i}$ es el coeficiente de difusión de la especie $i$.

Las aproximaciones de campo medio utilizadas son válidas para nanocanales cuyos radios son más grandes que dos veces la longitud de Debye (por ejemplo en una solución 0,1M, $R>2 n m)[17]$. La solución completa de las ecuaciones de NP- Navier-Stokes están en acuerdo cuantitativo con la aproximación de campo constante de Goldman en nanocanales cilíndricos cuya longitud es mayor a los 100 nanómetros, $L>100 \mathrm{~nm}[69]$. Se ha mostrado que las corrientes electro-osmóticas que se desprecian al reducir las ecuaciones de NP- Navier-Stokes a la aproximación de campo constante, tienen una contribución menor sobre la corriente total a través de nanocanales largos[69]. Se ha demostrado que la expresión 3.4 es válida para nano-

\footnotetext{
${ }^{1}$ La unidad de conductividad es siemens por metro y suele medirse a $T=25^{0} C$. La conductividad molar se mide en unidades de siemens por metro cuadrado sobre mol $\frac{S m^{2}}{\mathrm{~mol}}$.

2 http://uk.hach. com/conductivity-standard-kcl-0-1-m-12-88-ms-cm/product?id=24929257899

${ }^{3}$ Los valores que utilizamos para las mobilidades se encuentran en la tabla A.4
} 


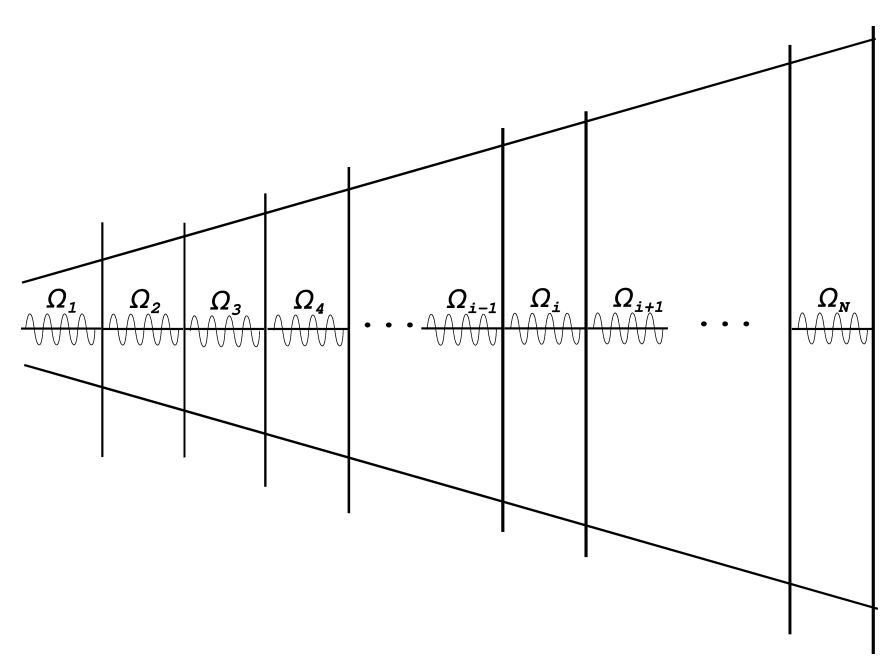

Figura 3.1.1: Esquema de la aproximación de un nanocanal de geometría no cilíndrica a partir de nanocanales cilíndricos. Modelo de resistencias conectadas en serie para un nanocanal de forma arbitraria.

canales cilíndricos cuyas dimensiones se encuentran en los rangos donde estas aproximaciones son válidas[66].

Geometrías más generales exhiben una respuesta no lineal frente al voltaje externo aplicado. Esta respuesta no lineal los define como sistemas no óhmicos, por ejemplo, los nanocanales asimétricos con carga en su superficie son de interés en potenciales aplicaciones de conversión de energía[76] por sus propiedades de rectificación de la corriente iónica. En estos casos resulta útil definir la conductancia de cuasi equilibrio como la pendiente de la curva $I$ vs $V$, en el límite $V \rightarrow 0$, ecuación 3.5 .

$$
G=\left.\frac{\partial I}{\partial V}\right|_{V=0}
$$

\subsubsection{Conductancia para distintas geometrías}

Para extender el cálculo de la conductancia de nanocanales cilíndricos a geometrías más generales consideramos dos aproximaciones. Por un lado, dada una geometría arbitraria la aproximamos por su versión discretizada. Dada una geometría donde el diámetro del nanocanal depende únicamente de la coordenada $z$, se la aproxima por la combinación de $N$ nanocanales cilíndricos conectados en serie ( ver figura 3.1.1). Este método es útil en geometrías que tienen simetría azimutal (homogéneos en la dirección angular $\hat{\theta}$ ) pero que pueden incorporar asimetrías en la dirección $\hat{z}$. Bajo esta aproximación consideramos que el i-ésimo nanocanal cilíndrico proveniente de la discretización de la geometría, tiene una resistencia $\Omega_{i}$. La resistencia total puede obtenerse como la suma algebraica de las $N$ resistencias en serie correspondientes a los $N$ nanocanales cilíndricos que aproximan la geometría, ecuación 3.6.

$$
\Omega_{t o t}=\sum_{i=1}^{N} \Omega_{i}
$$

Por otro lado, consideramos que la la i-ésima resistencia $\Omega_{i}$ correspondiente a un nanocanal puede aproximarse por la inversa de la conductancia $G_{i}$ proveniente de la expresión 3.4. En el límite del continuo la resistencia total se calcula integrando sobre la longitud del nanocanal, según la expresión 3.7, 


$$
\Omega_{t o t}=\int_{0}^{L} \frac{1}{G(z)} \frac{d z}{L}
$$

El cálculo de la resistencia total del sistema requiere conocer $G(z)$ a lo largo del eje del nanocanal. De la teoría molecular podemos conocer la conductancia $G$ para un nanocanal cilíndrico de diámetro $D$. A continuación escribimos la expresión 3.7 en términos de una integral respecto del diámetro del nanocanal. Dada una función $D(z)$ que describa la variación del diámetro del nanocanal con la posición a lo largo del eje principal $\hat{z}$, podemos relacionar la conductancia $G(D)$ con la resistencia total a través de la integral 3.7. La conductancia del nanocanal vendrá dada por la inversa de 3.7 ,

$$
G_{t o t}=\frac{1}{\Omega_{t o t}}=\frac{1}{\int_{0}^{L} \frac{1}{G(z)} \frac{d z}{L}}
$$

\section{Nanocanales con forma de Cono y Trompeta}

Como primera aproximación a nanocanales no cilíndricos se exploraron las geometrías cónica y tipo trompeta (perfil parabólico). Ambas geometrías se obtienen con la ecuación 3.9, donde $n=1$ corresponde a nanocanales cónicos y $n=2$ corresponde a nanocanales trompeta.

$$
r(z)=\Delta r\left(\frac{z}{L}\right)^{n}+R_{\text {min }}
$$

En estas geometrías se investigó la regulación de carga de polielectrolitos confinados (capítulo 5). La integración en la variable $z$ de la expresión 3.7 la escribimos en términos del diámetro. De acuerdo con la expresión que describe la geometría 3.9,

$$
\begin{gathered}
d r=\frac{n}{L}\left(R_{\max }-R_{\min }\right)\left[\frac{r-R_{\min }}{R_{\max }-R_{\min }}\right]^{(n-1) / n} d z \\
\frac{d z}{L}=\frac{1}{n} \frac{1}{\left(R_{\max }-R_{\min }\right)^{1 / n}} \frac{d r}{\left(r-R_{\min }\right)^{1-1 / n}}
\end{gathered}
$$

Con la relación 3.10 la expresión para la resistencia total del nanocanal cónico/trompeta queda,

$$
\Omega_{t o t}=\frac{1}{n} \frac{1}{\left(R_{\max }-R_{\min }\right)^{1 / n}} \int_{R_{\min }}^{R_{\max }} \frac{1}{G(D)\left(r-R_{\min }\right)^{1-1 / n}} d r
$$

Donde $n$ indica la forma del canal ( $n=1$ para conos, $n=2$ para nanocanales con forma de trompeta). Para calcular la resistencia total de un nanocanal cuya geometría es descripta por la ecuación 3.9, utilizamos la expresión 3.11 junto con la conductancia provista por la teoría molecular según la ecuación 3.4.

\section{Nanocanales con forma de bala ("Bullet-like nanochannels")}

Una geometría de interés experimental son las llamadas "bullet-like" o nanocanales con forma de bala.[8]. Estas geometrías son reproducibles y pueden obtenerse con procedimientos más o menos standard $[9,7,8]$. Estos procedimientos experimentales dan lugar a diversas geometrías[55] que pueden describirse según la ecuación 3.12,

$$
r(z)=\frac{R_{\max }-R_{\min } \exp \left\{-\left(\frac{L}{h}\right)^{n}\right\}-\Delta \exp \left\{-\left(\frac{z}{L}\right)^{n}\left(\frac{L}{h}\right)^{n}\right\}}{1-\exp \left\{-\left(\frac{L}{h}\right)^{n}\right\}}
$$




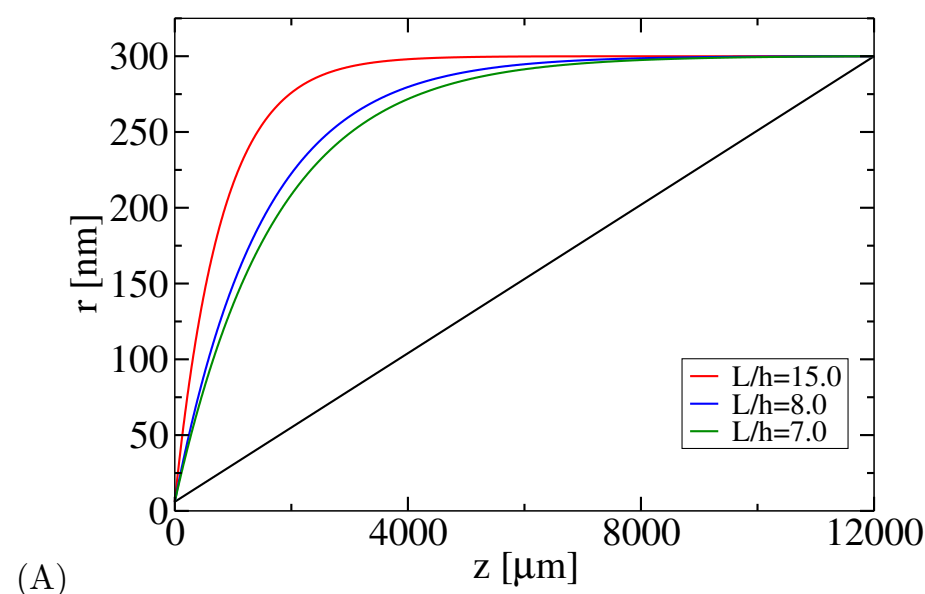

(A)

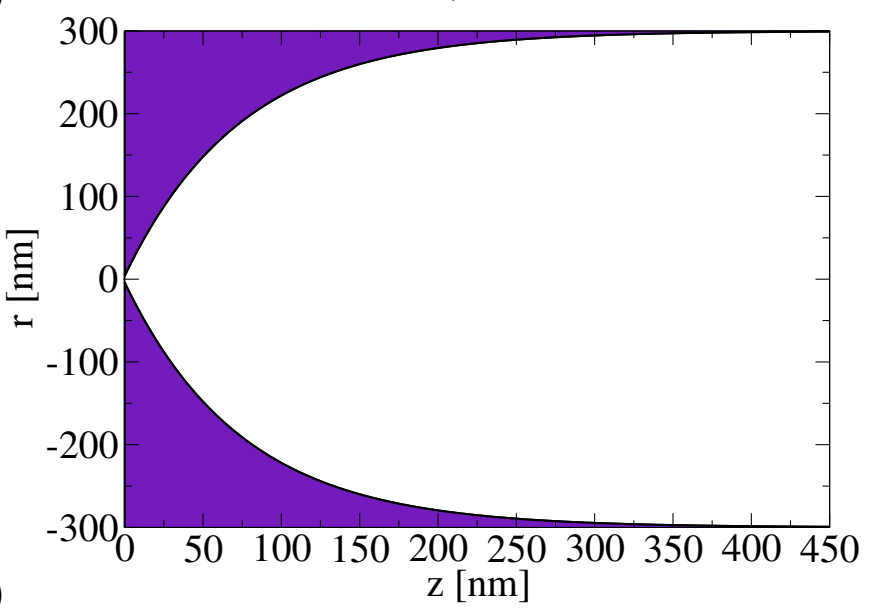

Figura 3.1.2: (A) Comparación de la geometría "bullet-like" para distintos valores del parámetro de forma $h . L=12 \mu m, R_{\max }=300 \mathrm{~nm}, R_{\min }=10 \mathrm{~nm}$. (B) Ejemplo del perfil de un nanocanal "bullet-like".

esta ecuación considera los radios mínimo $R_{\min }$ y máximo $R_{\max }$, la longitud del nanocanal $L$ y dos parámetros de forma $n$ y $h$. La familia de geometrías "bullet-like" se caracteriza por tener $n=1$. En la figura 3.1.2, se muestran los cambios en la geometría debido al parámetro $h$. Tras un reordenamiento de los términos podemos expresar la dependencia del radio con la coordenada $z$ como

$$
r(z)=R_{\min }+\Delta r\left[\frac{1-e^{-\left(\frac{z}{L}\right)\left(\frac{L}{h}\right)}}{1-e^{-\left(\frac{L}{h}\right)}}\right]
$$

y la relación entre los diferenciales $d z$ y $d r$, como

$$
\frac{d z}{L}=\frac{1}{(L / h)} \frac{\left(1-e^{-\left(\frac{L}{h}\right)}\right)}{1-\left(1-e^{-\left(\frac{L}{h}\right)}\right) \frac{\left(r-R_{\min }\right)}{\Delta r}} \frac{d r}{\Delta r}
$$

Esto nos permite reescribir la resistencia total incorporando la información geométrica en la ecuación 3.7,

$$
\Omega^{(L / h)}=\left(\frac{h}{L}\right) \frac{\left(1-e^{-\left(\frac{L}{h}\right)}\right)}{\Delta r} \int_{R_{\min }}^{R_{\max }} \frac{1}{G(r)} \frac{1}{1-\left(1-e^{-\left(\frac{L}{h}\right)}\right) \frac{\left(r-R_{\min }\right)}{\Delta r}} d r
$$

La expresión 3.15 representa la resistencia total de un nanocanal "bullet-like" expresada en 


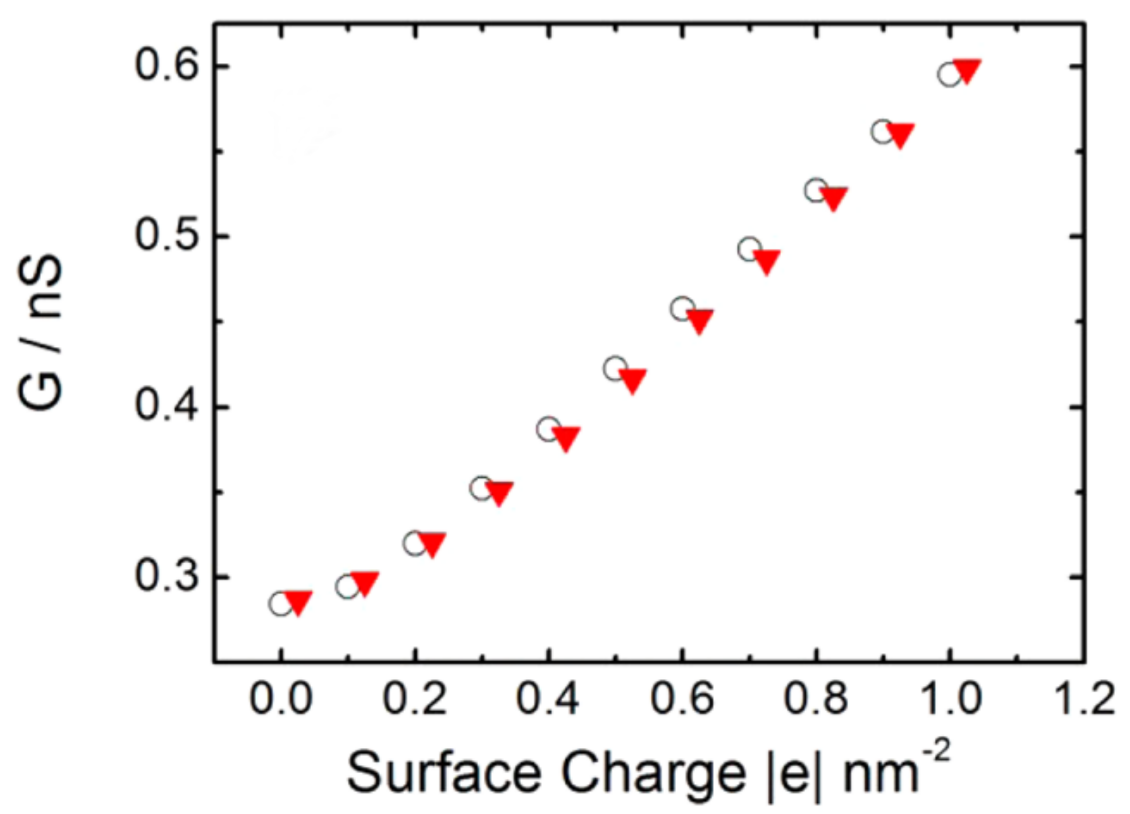

Figura 3.1.3: Comparación de la conductancia de un nanocanal cónico $\left(D_{\min }=10 \mathrm{~nm}\right.$, $\left.D_{\max }=290 \mathrm{~nm}, L=12 \mu \mathrm{m}\right)$ como función de la carga superficial. Los círculos muestran la conductancia obtenida con nuestra metodología teórica y los triángulos muestran la solución de las ecuaciones de Poisson-Nernst-Planck obtenida por cálculo de elementos finitos, ver la información suplementaria de la referencia [67].

términos de la conductancia de nanocanales cilíndricos.

\section{Sobre la calidad de la aproximación}

La conductancia obtenida como la inversa de la resistencia para geometrías no cilíndricas fue comparada con resultados obtenidos por otras técnicas. Primero consideramos un nanocanal cónico de dimensiones: $D_{\min }=10 \mathrm{~nm}, D_{\max }=290 \mathrm{~nm}, \mathrm{~L}=12 \mu \mathrm{m}$, con carga superficial. Los resultados de conductancia (inversa de la expresión 3.11 para el caso cónico, $n=1$ ), fueron comparados con los resultados obtenidos luego de resolver las ecuaciones de Poisson-Nernst-Planck por elementos finitos mediante el software COMSOL, figura 3.1.3. Ambas curvas muestran un comportamiento similar de la conductancia, aproximadamente lineal con la densidad de carga superficial.

En segundo lugar consideramos los resultados de la caracterización experimental de un nanocanal con geometría "bullet-like" mediante curvas $I$ vs $V$. Los resultados experimentales corresponden a un nanocanal con geometría "bullet-like" y con grupos carboxilato $(\mathrm{COOH})$ sobre la superficie. La constante de equilibrio ácido-base utilizada para los grupos carboxilatos sobre la pared del nanocanal es $p K a_{\text {wall }}=4,5 . \mathrm{A} \mathrm{pH}=2.0$, todos los grupos $C O O H$ están protonados (en estado neutro) por lo tanto la carga neta en superficie es cero. El valor que predice la teoría para la conductancia del canal sin cargas superficiales es $6,88 n \mathrm{n}$ en completo acuerdo con los resultados experimentales que arrojan un valor de $6,90 n S$ cuando no hay carga superficial. Este valor se obtiene para un diámetro menor de $D_{\min }=12 n m$ y un factor de forma $\frac{L}{h}=16$. En el extremo de valores altos de $\mathrm{pH}, \mathrm{pH}=9$, los grupos $\mathrm{COOH}$ están completamente deprotonados y por lo tanto existe carga superficial en el nanocanal. La consideración de la carga superficial se realiza a través del parámetro $\sigma_{q}$ que representa el número de cargas por unidad de superficie $\frac{e}{n m^{2}}$, es decir se asume que la carga superficial esta distribuida uniformemente dentro del canal. De la comparación con los datos experimentales, se obtiene que la carga superficial en la pared del nanocanal es $\sigma_{q}=0,4\left[\frac{e}{n m^{2}}\right]$. En la figura 3.1.4, mostramos la conductancia 


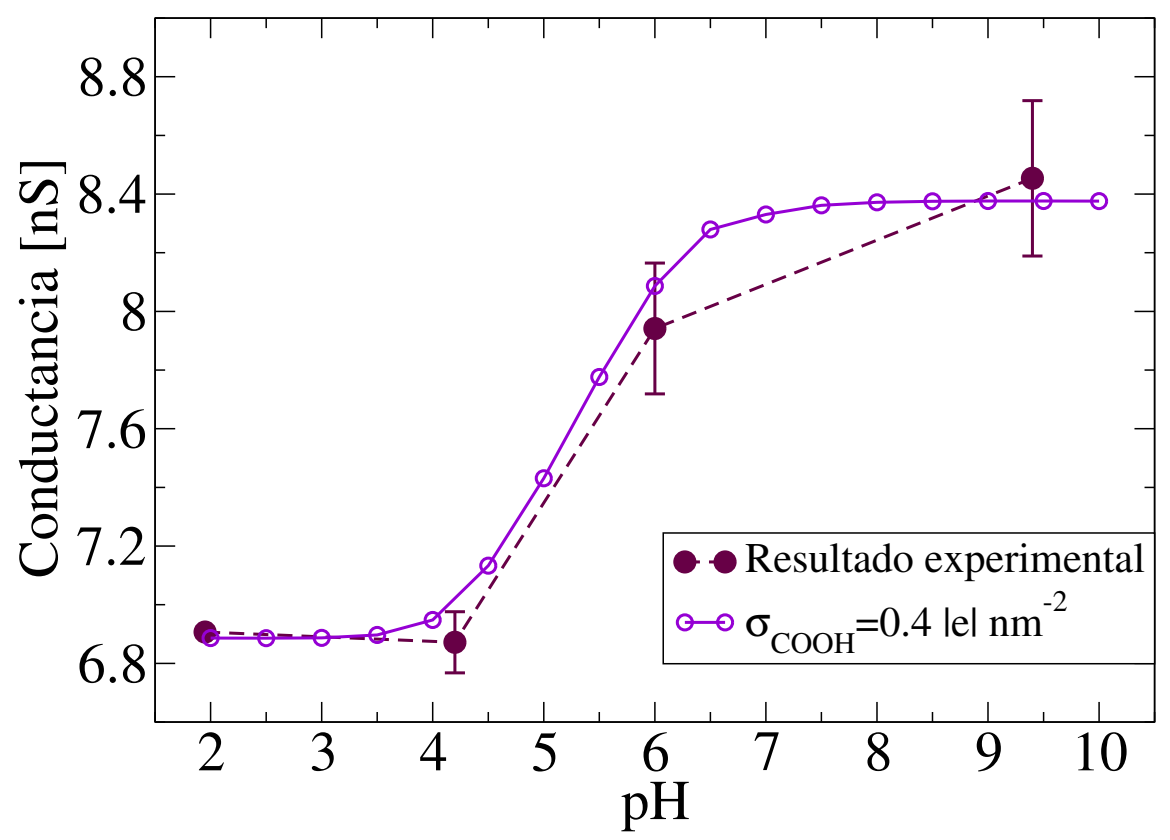

Figura 3.1.4: Comparación de la conductancia de un nanocanal "bullet-like" con carga superficial dependiente del pH de la solución. Los datos experimentales fueron medidos en un $\left(D_{\min }=12 \mathrm{~nm}, D_{\max }=600 \mathrm{~nm}, \mathrm{~L}=12 \mu \mathrm{m}\right)$ como función del pH. Los resultados experimentales corresponden a un nanocanal con forma "bullet-like" con grupos $C O O H$ en la superficie. A $\mathrm{pH}=2.0$, se puede asumir que todos los grupos $C O O H$ están protonados y por lo tanto la carga neta en superficie es cero. En la figura, se muestran los puntos experimentales junto con las curvas obtenidas con teoría molecular.

de un nanocanal tipo con sitios responsivos al pH de la solución. Las conductancias calculadas mediante la expresión 3.15 en función del pH de la solución están en completo acuerdo con los resultados experimentales. La caracterización geométrica $D_{\min }=12 \mathrm{~nm}, \frac{L}{h}=16$ y la densidad de carboxilatos en la superficie interna $\sigma_{q}=0,4\left[\frac{e}{n m^{2}}\right]$ están en completo acuerdo con lo reportado por Apel et.al [8]. Los parámetros geométricos que mejor caracterizan estos nanocanales de geometría "bullet-like" fueron calculados mediante la resolución de las ecuaciones de $\mathrm{PNP}[8]$ y se obtuvieron los siguientes rangos de validez: densidad de carboxilatos en superficie $\sigma_{q} \in[0,2 ; 0,5] \mathrm{e} / \mathrm{nm}^{2}$, parámetro geométrico $\frac{L}{h} \in[14 ; 21]$.

Nuestro método de estimación para la conductividad iónica arroja valores compatibles con los resultados obtenidos mediante métodos experimentales y computacionales.

Para que la aproximación por cilindros sea compatible con la teoría molecular debemos pedir que las las propiedades físicas y químicas del sistema no varíen entre dos cilindros adyacentes. Para ello pedimos que el cambio en el radio sea mucho menor que el radio local del nanocanal. Imponemos la condición 3.16,

$$
\frac{R_{i+1}-R_{i}}{R_{i}} \ll 1
$$

Si consideramos el contorno $D(z)$ del nanocanal, la derivada del contorno permite relacionar el cambio en el radio con la discretización del canal. Por ejemplo, para el caso de una geometría "bullet-like"

$$
\frac{d a}{d z}=\frac{\Delta r}{\left(1-e^{-\left(\frac{L}{h}\right)}\right)} \frac{1}{h} e^{-\left(\frac{z}{L}\right)\left(\frac{L}{h}\right)} \leq \frac{\Delta r}{\left(1-e^{-\left(\frac{L}{h}\right)}\right) h}<0,4
$$

si $L=12000 \mathrm{~nm}, D_{\min }=12 \mathrm{~nm}, D_{\max }=600 \mathrm{~nm}, L / h=16$ y por lo tanto $h=750 \mathrm{~nm}$, la 
pendiente local de la geometría está acotada por el valor 0,4 .

$$
\frac{\Delta z_{i}}{R_{i}} \frac{\Delta R_{i}}{\Delta z_{i}}<\frac{\Delta z_{i}}{R_{i}} 0,4 \ll 1
$$

La discretización en nanocanales cilíndricos debe ser tal que $\Delta z_{i}<2,5 \times R_{i}$.

\section{Valor Medio de una Propiedad $E(r, z)$ para una Geometría arbitraria}

La discretización de la geometría del nanocanal en nanocanales cilíndricos introduce la posibilidad de estudiar las propiedades promedio dentro de un nanocanal de forma no cilíndrica. El valor medio $(\langle E\rangle)$ de una propiedad $E(r, z)$ que depende de la posición dentro del nanocanal es calculado a través del promedio en las direcciones radial $\hat{r}$ y axial $\hat{z}$. Por definición

$$
\overline{\langle E\rangle}=\frac{\int \rho_{p}(\boldsymbol{r}) E(\boldsymbol{r}) d \boldsymbol{r}}{\int \rho_{p}(\boldsymbol{r}) d \boldsymbol{r}}
$$

donde $\rho_{p}(\boldsymbol{r})$ es la densidad de polímeros en la posición vectorial $\boldsymbol{r}$. Asumimos que el sistema es homogéneo en la coordenada angular, por lo que $E$ y $\rho_{P}$ no dependen de $\theta$. El denominador representa la cantidad total de polímero dentro del nanocanal. Si definimos $\sigma$ como la densidad superficial de cadenas poliméricas, el número total de segmentos poliméricos en una porción cilíndrica de anchoa $d z$ es,

$$
\int_{0}^{R} \int_{0}^{2 \pi} \rho_{p}(r) r d \theta d r d z=2 \pi R \sigma N d z
$$

la integración sobre la coordenada $z$ nos devuelve,

$$
\begin{aligned}
\int_{0}^{L} \int_{0}^{R(z)} \int_{0}^{2 \pi} \rho_{p}(r, z) r d \theta d r d z & =2 \pi \sigma N \int_{0}^{L} R(z) d z \\
& =2 \pi \sigma N L\left(\frac{\Delta R}{n+1}+R_{\min }\right)
\end{aligned}
$$

Para la última expresión se utilizó la descripción geométrica polinomial 3.9. A continuación relacionamos el valor medio parcial dentro de una porción cilíndrica que denotaremos con $\langle E\rangle^{\prime}$ con el valor medio total dentro de un nanocanal $(\langle E\rangle)$. La expresión para el valor medio de una propiedad $E(\boldsymbol{r})$ en el caso de una porción cilíndrica es,

$$
\langle E\rangle^{\prime}(z)=\frac{\int_{0}^{R(z)} \int_{0}^{2 \pi} \rho_{p}(r, z) E(r, z) r d \theta d r}{\int_{0}^{R(z)} \int_{0}^{2 \pi} \rho_{p}(\boldsymbol{r}) r d \theta d r}=\frac{\int_{0}^{R(z)} \rho_{p}(r, z) E(r, z) r d r}{\int_{0}^{R(z)} \rho_{p}(\boldsymbol{r}) r d r}
$$

usando esta última ecuación, el valor $\langle E\rangle^{\prime}$ queda determinado a partir de los cálculos de teoría molecular en sistemas donde las inhomogeneidades dependan sólo de la variable $r$ (Cálculos en 1D). Si combinamos las ecuaciones $3.18,3.19$ y 3.20 , para relacionar $\langle E\rangle$ y $\langle E\rangle^{\prime}$, obtenemos

$$
\langle E\rangle=\int_{0}^{L}\langle E\rangle^{\prime}(z) r(z) d z \frac{1}{L\left(\frac{\Delta R}{n+1}+R_{\min }\right)}
$$

como ejemplo, se dejan las expresiones para el valor promedio del grado de disociación y la carga promedio por monómero de un nanocanal no-cilíndrico, expresiones 3.21 y 3.22 , respectivamente. 


$$
\begin{gathered}
\left\langle f_{i}\right\rangle^{(n)}=\int_{0}^{L}\left\langle f_{i}\right\rangle^{\prime}(z) R(z) d z \frac{1}{L\left(\frac{\Delta R}{n+1}+R_{\text {min }}\right)} \\
\left\langle Q_{\text {mon }}\right\rangle^{(n)}=\int_{0}^{L}\left\langle Q_{\text {mon }}\right\rangle^{\prime}(z) R(z) d z \frac{1}{L\left(\frac{\Delta R}{n+1}+R_{\text {min }}\right)}
\end{gathered}
$$




\section{Capítulo 4}

\section{Adsorción de Polielectrolitos Débiles en Medios Confinados}

La adsorción de polielectrolitos sobre la superficie interna de los nanocanales es un método alternativo para la preparación de nanocanales modificados por polímeros que resulta sintéticamente más simple que la polimerización de brushes. Este fenómeno es posible gracias a la existencia de cargas en la superficie interna del nanocanal y una interacción atractiva (interacción de Van der Waals) entre los monómeros del polielectrolito y la pared del nanocanal. La adsorción resulta favorable por la liberación de los contraiones de la pared y del polímero más que por la atracción electrostática entre ellos (es un proceso entrópico, no entálpico). La carga superficial en la pared del canal se debe a la presencia de grupos carboxilatos que se presuponen distribuidos de forma homogénea sobre la pared interna del nanocanal.

Para la modelización teórica es frecuente encontrar en la literatura trabajos donde se considera que luego de la adsorción del polielectrolito, la densidad de carga superficial es homogénea a lo largo de la pared interna del nanocanal. Esta hipótesis es razonable en el caso de nanocanales cilíndricos debido a la simetría del nanocanal. Sin embargo, cuando la geometría del nanocanal no es cilíndrica, por ejemplo geometrías asimétricas como nanocanales cónicos o geometrías tipo bala (bullet-like), podrían aparecer otro tipo de distribuciones.

Aunque existen experimentos donde es posible estimar la carga superficial dentro del nanocanal, actualmente no es posible medir si la adsorción del polielectrolito depende de la curvatura del nanocanal. Los esfuerzos teórico/computacionales de la comunidad de los últimos años se orientaron hacia la descripción de las propiedades de transporte de estos sistemas más que a la organización molecular. Más específicamente a la medición y predicción de las propiedades de rectificación.

En este capítulo estudiamos la adsorción de cadenas poliméricas en medios confinados, ver figura 4.0.1(A). Para ello consideramos un nanocanal cuyas dimensiones radiales son menores a su largo, $L \gg R$. Consideramos que el sistema alcanza el equilibrio con un entorno que tiene polielectrolitos "libres", en una concentración $C_{p o l}$ en solución. Dos nuevos términos deben incorporarse a la energía libre del sistema, un término correspondiente a la entropía traslacional de las cadenas poliméricas y otro correspondiente al potencial químico de las cadenas poliméricas $\mu_{p o l}$. Consideramos además una interacción de Van der Waals entre los monómeros del polielectrolito (PAH) y la pared del nanocanal. La condición de equilibrio termodinámico para los polielectrolitos implica que el potencial químico de las cadenas libres debe igualarse al potencial químico de las cadenas adsorbidas[23]. Cada monómero de la cadena posee un equi- 

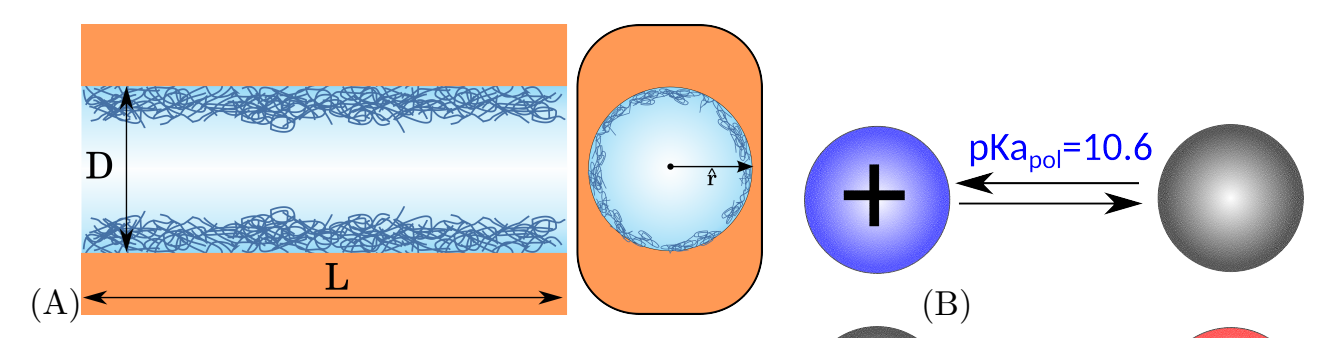

(B)
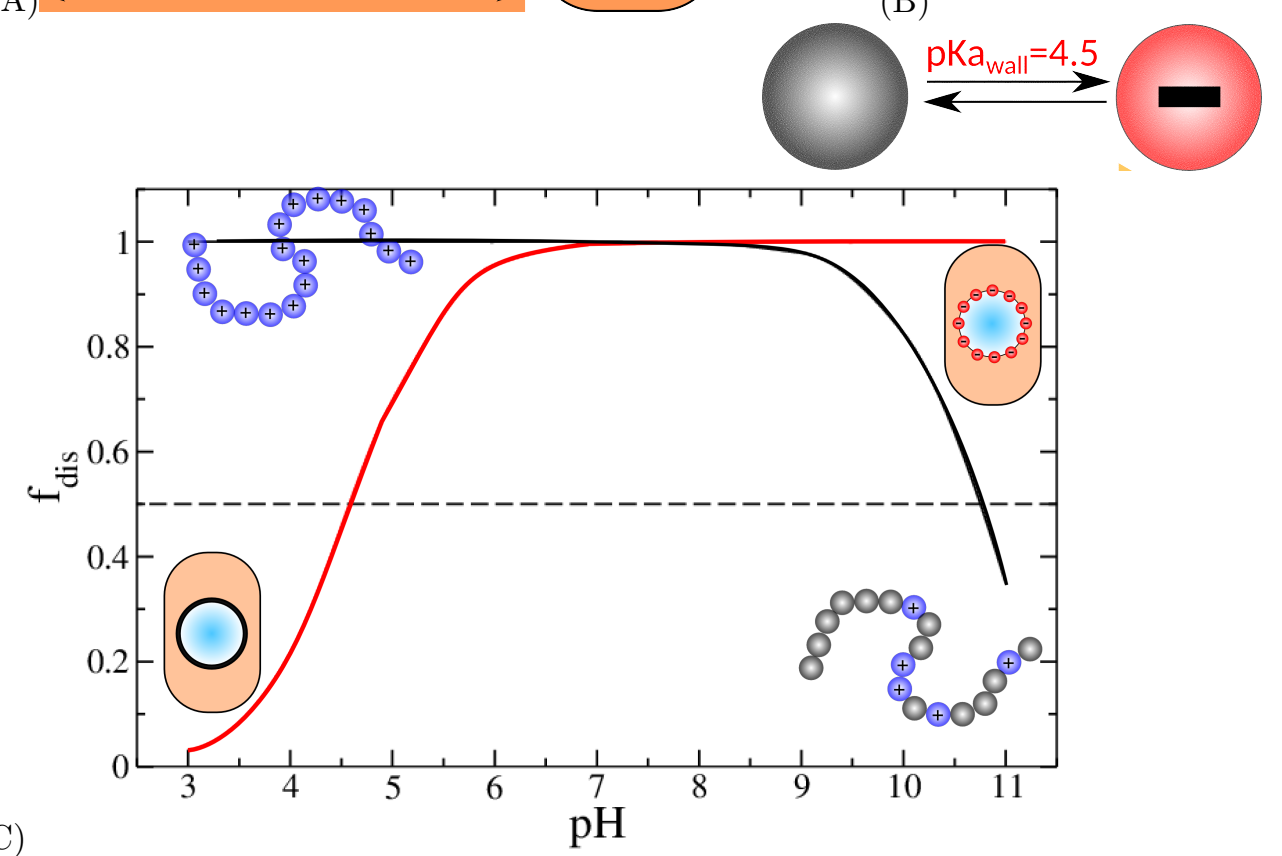

Figura 4.0.1: El sistema en estudio es un nanocanal cilíndrico modificado con una capa de PAH. A) Muestra una vista longitudinal y transversal del nanocanal cilíndrico. B) Muestra los equilibrios químicos del monómero de PAH y del grupo carboxilato presente en la pared del nanocanal. C) Gráfico del grado de disociación promedio del polímero y la pared como función del $\mathrm{pH}$ de la solución.

librio químico simple, ver figura 4.0.1(B), donde los estados del monómero son dos: con carga nula o carga neta $+1 e$. Sobre la superficie interna del nanocanal existen grupos carboxilatos que también poseen un equilibrio químico simple que conecta dos estados: carga nula 0 y carga $-1 e$, ver figura 4.0.1(B).

Finalmente se estudia el caso de adsorción en nanocanales no-cilíndricos. En particular estudiamos un ejemplo de nanocanales asimétricos, nanocanales cónicos de amplio interés en la comunidad por sus propiedades de rectificación del flujo iónico. Consideraremos simetría en la dirección $\hat{\theta}$ e incorporamos cambios en el diámetro del nanocanal a lo largo de la dirección $\hat{z}$.

\subsection{Energía libre del sistema}

La adsorción de polielectrolitos débiles sobre la pared interna de nanocanales cilíndricos implica un cambio en la descripción teórica presentada en el capítulo 2. En este sistema debemos considerar cadenas poliméricas libres por lo que la descripción de las conformaciones estará dada por un par $(\boldsymbol{r}, \alpha)$, donde $\alpha$ indica la conformación y una coordenada espacial $\boldsymbol{r}$ indica la posición en la que se encuentra dicha conformación.

Decimos que una conformación $\alpha$ se encuentra en la posición $\boldsymbol{r}$, si $\boldsymbol{r}$ es la posición del monómero de la cadena más alejado del eje principal del nanocanal (eje $\hat{z}$ ). En analogía con los términos de la ecuación 2.11, escribimos la entropía de mezcla de cadenas libres como, 


$$
-T S_{m i x-P o l .}(\boldsymbol{r})=k_{B} T \rho_{P C}(\boldsymbol{r})\left[\ln \left(\rho_{P C}(\boldsymbol{r}) v_{w}\right)-1\right]
$$

donde $\rho_{P C}(\boldsymbol{r})$ es la densidad de cadenas en $\boldsymbol{r}$. La densidad de cadenas es necesaria para poder escribir el término entrópico de mezcla. La densidad local de monómeros está relacionada con la densidad de cadenas a través de la ecuación 4.2

$$
\left\langle\rho_{P}(\boldsymbol{r})\right\rangle=\rho_{P C}(\boldsymbol{r}) \int d \boldsymbol{r}^{\prime} \sum_{\alpha} n_{p}\left(\boldsymbol{r} ; \boldsymbol{r}^{\prime}, \alpha\right) P\left(\boldsymbol{r}^{\prime}, \alpha\right)
$$

donde $n_{p}\left(\boldsymbol{r} ; \boldsymbol{r}^{\prime}, \alpha\right)$ es la densidad de monómeros en la posición $\boldsymbol{r}$, debido a la conformación $\left(\boldsymbol{r}^{\prime}, \alpha\right)$, ver apéndice E. El caso más general de entropía conformacional de cadenas poliméricas libres se escribe como,

$$
-T S_{p o l}=\int k_{B} T \rho_{P C}(\boldsymbol{r}) \sum_{\alpha} P(\boldsymbol{r}, \alpha) \ln (P(\boldsymbol{r} ; \alpha)) d \boldsymbol{r}
$$

donde $P(\boldsymbol{r}, \alpha)$ es la función densidad de probabilidad de la configuración $(\boldsymbol{r}, \alpha)$. Formalmente debemos incorporar también la normalización de la probabilidad de las conformaciones de cadena $P(\boldsymbol{r}, \alpha)$. Para ello vamos a considerar la normalización local, es decir vamos a pedir que la probabilidad $P(\boldsymbol{r}, \alpha)$ cumpla con la ecuación 4.4.

$$
\sum_{\alpha} P(\boldsymbol{r}, \alpha)=1 \quad \text { para cada } \boldsymbol{r}
$$

Como el sistema esta en equilibrio con un entorno que contiene polielectrolitos libres en solución, debemos considerar que la cantidad de cadenas del polielectrolito dentro del canal estará regulada por el potencial químico del polímero $\mu_{p o l}$. La expresión general de la energía queda de la forma 


$$
\begin{aligned}
& \omega=\int_{0}^{R} \rho_{C l^{-}}(r)\left[\ln \left(\rho_{C l^{-}}(r) v_{w}\right)-1\right]\left(\frac{r}{R}\right) d r+\int_{0}^{R} \rho_{K^{+}}(\boldsymbol{r})\left[\ln \left(\rho_{K^{+}}(\boldsymbol{r}) v_{w}\right)-1\right]\left(\frac{r}{R}\right) d r \\
& +\int_{0}^{R} \rho_{O H^{-}}(r)\left[\ln \left(\rho_{O H^{-}}(r) v_{w}\right)-1\right]\left(\frac{r}{R}\right) d r+\int_{0}^{R} \rho_{H^{+}}(r)\left[\ln \left(\rho_{H^{+}}(r) v_{w}\right)-1\right]\left(\frac{r}{R}\right) d r \\
& +\int_{0}^{R} \rho_{w}(r)\left[\ln \left(\rho_{w}(r) v_{w}\right)-1\right]\left(\frac{r}{R}\right) d r+\int_{0}^{R} \rho_{P C}(r)\left[\ln \left(\rho_{P C}(r) v_{w}\right)-1\right]\left(\frac{r}{R}\right) d r \\
& +\int_{0}^{R} \rho_{P C}(r) \sum_{\alpha} P(r, \alpha) \ln (P(r ; \alpha))\left(\frac{r}{R}\right) d r-\mu_{p o l} \int_{0}^{R} \rho_{P C}(r)\left(\frac{r}{R}\right) d r \\
& +\int_{0}^{R}\left\langle\rho_{P}(r)\right\rangle\left\{\sum _ { e = 1 } ^ { m } f _ { e } ( r ) \left[\ln \left(\frac{f_{e}(r)}{f_{0}(r)}\right)-\sum_{n=1}^{e} \ln \left(K_{n}\right)-\left(n_{H^{+}, 0}-n_{H^{+}, e}\right) \beta \mu_{H^{+}}^{0}\right.\right. \\
& \left.\left.-\left(n_{O H^{-}, 0}-n_{O H^{-}, e}\right) \beta \mu_{O H^{-}}^{0}\right]+\ln \left(f_{0}(r)\right)+\beta \mu_{0}^{0}\right\}\left(\frac{r}{R}\right) d r \\
& -\int_{0}^{R} \beta \chi_{\text {wall }}(r)\left\langle\rho_{P}(r)\right\rangle\left(\frac{r}{R}\right) d r+\int_{0}^{R} \beta\left[\left\langle\rho_{q}(r)\right\rangle \Psi(r)-\frac{1}{2} \varepsilon\left(\nabla_{r} \Psi(r)\right)^{2}\right]\left(\frac{r}{R}\right) d r \\
& +\frac{1}{2} \int_{0}^{R} \int_{0}^{R} d r^{\prime}\left(2 \pi r^{\prime}\right) \chi\left(r-r^{\prime}\right)\left\langle\phi_{p}(r)\right\rangle\left\langle\phi_{p}\left(r^{\prime}\right)\right\rangle\left(\frac{r}{R}\right) d r \\
& -\int_{0}^{R} \beta\left\{\mu_{w} \rho_{w}(r)+\mu_{C l^{-}} \rho_{C l^{-}}(r)+\mu_{K^{+}} \rho_{K^{+}}(r)\right. \\
& +\mu_{O H^{-}}\left(\rho_{O H^{-}}(r)+\left[\sum_{e=1}^{m}\left(n_{e, O H^{-}}-n_{0, O H^{-}}\right) f_{e}(r)+n_{0, O H^{-}}\right]\left\langle\rho_{p}(r)\right\rangle\right) \\
& \left.+\mu_{H^{+}}\left(\rho_{H^{+}}(r)+\left[\sum_{e=1}^{m}\left(n_{e, H^{+}}-n_{0, H^{+}}\right) f_{e}(r)+n_{0, H^{+}}\right]\left\langle\rho_{p}(r)\right\rangle\right)\right\}\left(\frac{r}{R}\right) d r \\
& +\int_{0}^{R} \beta \pi(r)\left[\left\langle\phi_{P}(r)\right\rangle+\sum_{i} \phi_{i}(r)-1\right]\left(\frac{r}{R}\right) d r+\int_{0}^{R} \beta \lambda\left[\sigma_{q} f_{\text {wall }}+\int d r \beta\left\langle\rho_{q}(r)\right\rangle\right]\left(\frac{r}{R}\right) d r \\
& +\int_{0}^{R} \beta Z(r)\left(\sum_{\alpha} P(r, \alpha)-1\right)\left(\frac{r}{R}\right) d r
\end{aligned}
$$

Luego de reordenar los términos obtenemos en coordenadas cilíndricas, 


$$
\begin{aligned}
& \omega=\int_{0}^{R} \rho_{C l^{-}}(r)\left[\ln \left(\rho_{C l^{-}}(r) v_{w}\right)-1-\beta \mu_{C l^{-}}+\beta \pi(r) v_{C l^{-}}\right]\left(\frac{r}{R}\right) d r \\
& +\int_{0}^{R} \rho_{K^{+}}(r)\left[\ln \left(\rho_{K^{+}}(r) v_{w}\right)-1-\beta \mu_{K^{+}}+\beta \pi(r) v_{K^{+}}\right]\left(\frac{r}{R}\right) d r \\
& +\int_{0}^{R} \rho_{O H^{-}}(r)\left[\ln \left(\rho_{O H^{-}}(r) v_{w}\right)-1-\beta \mu_{O H^{-}}+\beta \pi(r) v_{O H^{-}}\right]\left(\frac{r}{R}\right) d r \\
& +\int_{0}^{R} \rho_{H^{+}}(r)\left[\ln \left(\rho_{H^{+}}(r) v_{w}\right)-1-\beta \mu_{H^{+}}+\beta \pi(r) v_{H^{+}}\right]\left(\frac{r}{R}\right) d r \\
& +\int_{0}^{R} \rho_{w}(r)\left[\ln \left(\rho_{w}(r) v_{w}\right)-1-\beta \mu_{w}+\beta \pi(r) v_{w}\right]\left(\frac{r}{R}\right) d r \\
& +\int_{0}^{R} \rho_{P C}(r)\left[\ln \left(\rho_{P C}(r) v_{w}\right)-1-\beta \mu_{p o l}+\beta \pi(r) v_{p}+\sum_{\alpha} P(r, \alpha) \ln (P(r ; \alpha))\right]\left(\frac{r}{R}\right) d r \\
& +\int_{0}^{R}\left\langle\rho_{P}(r)\right\rangle\left\{\sum _ { e = 1 } ^ { m } f _ { e } ( r ) \left[\ln \left(\frac{f_{e}(r)}{f_{0}(r)}\right)-\sum_{n=1}^{e} \ln \left(K_{n}\right)-\left(n_{e, H^{+}}-n_{0, H^{+}}\right) \beta \Delta \mu_{H^{+}}\right.\right. \\
& \left.-\left(n_{e, O H^{-}}-n_{0, O H^{-}}\right) \beta \Delta \mu_{o H^{-}}\right]-n_{0, O H^{-}} \beta \mu_{O H^{-}}-n_{0, H^{+}} \beta \mu_{H^{+}} \\
& \left.+\ln \left(f_{0}(r)\right)+\beta \mu_{0}^{0}-\beta \chi_{\text {wall }}(r)\right\}\left(\frac{r}{R}\right) d r \\
& +\int_{0}^{R} \beta\left[\left\langle\rho_{q}(r)\right\rangle \Psi(r)-\frac{1}{2} \varepsilon\left(\nabla_{r} \Psi(r)\right)^{2}\right]\left(\frac{r}{R}\right) d r-\int_{0}^{R} \beta \pi(r)\left(\frac{r}{R}\right) d r \\
& +\int_{0}^{R} \beta Z(r)\left(\sum_{\alpha} P(r, \alpha)-1\right)\left(\frac{r}{R}\right) d r
\end{aligned}
$$

Donde $Z(r)$ es el multiplicador de Lagrange que impone la condición de normalización de $P(r, \alpha)$ de forma local. Por ello su dependencia con la coordenada radial. La densidad de monómeros local en función de las probabilidades de cadena 4.7.

$$
\left\langle\rho_{P}(r)\right\rangle=\int_{0}^{R} \rho_{P C}\left(r^{\prime}\right) \sum_{\alpha} P\left(r^{\prime}, \alpha\right) n\left(r ; r^{\prime}, \alpha\right) d r^{\prime}
$$

Uno de los resultados más importantes es el que se obtiene después de optimizar la funcional 4.6 respecto de la densidad de cadenas de polielectrolito, el potencial químico del polímero:

$$
\beta \mu_{P}=\ln \left(\rho_{P C}(r) v_{w}\right)-\ln (Q(r))
$$

Como consideramos que todas las cadenas están adsorbidas en la superficie interna del nanocanal, es posible definir una densidad superficial de cadenas $\sigma_{\text {pol }}$ cuya relación con la densidad $\rho_{P C}(R)$ es:

$$
\rho_{P C}(R)=\rho_{P C}=\frac{\sigma_{p o l}}{\delta}
$$

en esta definición se asume que todas las cadenas que están a menos de una distancia $\delta$ de la superficie están efectivamente unidas a la misma. En este trabajo usamos $\delta=0,5 n m$, que es equivalente a la longitud de un segmento del polímero en el modelo molecular utilizado. La elección de $\delta$ implica simplemente un termino aditivo fijo entre la diferencia de los potenciales químicos del polímero en solución y en superficie, es decir no modificara nuestros resultados en forma cualitativa. 


\subsection{Adsorción en nanocanales cilíndricos}

Por consideraciones geométricas sabemos que la distribución de polímero en nanocanales cilíndricos largos debe ser homogénea a lo largo de la dirección $\hat{z}$ del nanocanal. En nanocanales largos, la cantidad total de polímero adsorbido depende de la curvatura y la carga superficial de la pared del nanocanal. Estamos interesados en la carga superficial que experimentaría un ion libre al atravesar un nanocanal modificado con polielectrolitos. Por ello, definimos la densidad de carga superficial efectiva $\sigma_{e f f}$, como la suma de la densidad de cargas proveniente de las cadenas de polielectrolitos en la superficie $\left(N \sigma_{\text {pol }} f_{1}\right)$ más la densidad de carga superficial presente en la pared $\left(\sigma_{q} f_{w}\right)$. Para calcular $\sigma_{p o l}$ se contaron el número de cadenas con al menos 1 monómero sobre la superficie del nanocanal (a una distancia menor a $\delta$ de la superficie). En los gráficos de la figura 4.2.1, mostramos $\sigma_{\text {eff }}$ (sobre el eje de la izq.) y la densidad superficial de monómeros de cadenas pegadas a la superficie de la pared, $N \sigma_{\text {pol }}$ (sobre el eje de la der.), los gráficos están en función del diámetro del nanocanal. Utilizamos $\mathrm{pH}=7.0$ porque los grupos carboxilatos sobre la pared y los monómeros del PAH están, ambos, completamente cargados (ver figura 4.0.1), y una densidad de carga sobre la pared de $\sigma_{q}=0,1 e / \mathrm{nm}^{2}$.

La densidad de carga superficial efectiva $\sigma_{e f f}$ puede aumentar o disminuir con el diámetro del nanocanal dependiendo de las diferentes concentraciones de polielectrolitos $\left(C_{p o l}\right)$ y de la sal en solución $\left(C_{\text {salt }}\right)$. En determinadas condiciones incluso se observa una inversión en el signo de la carga efectiva, es decir, las cargas del polielectrolito adsorbido sobrecompensan a las presentes en la pared.

Las curvas en la figura 4.2.1A corresponden a una concentración de sal en solución de $C_{\text {salt }}=0,1 M$. Se observa que el comportamiento de $\sigma_{\text {eff }}$ en función del diámetro del canal cambia con la concentración de polielectrolitos en solución $\left(C_{p o l}\right)$. Cuando $C_{p o l}=10^{-3} \mathrm{M}$ la densidad de carga efectiva tiene signo negativo $\sigma_{\text {eff }}<0\left(\sigma_{\text {eff }} \sim-0,03 e \times n m^{-2}\right)$ y es independiente del diámetro del canal. Si $C_{p o l}>1 \mathrm{mM}$ entonces la densidad de polielectrolitos adsorbidos aumenta al aumentar el diámetro del nanocanal, además, se observa una inversión en el signo de la carga efectiva para alguna combinación de $C_{p o l}$ y $D$. Como la densidad de carga de la pared es constante el cambio de signo se debe exclusivamente a la adsorción de los polielectrolitos. Cuando $\sigma_{e f f}>0$ la carga de las cadenas adsorbidas es mayor que la carga proveniente de los grupos carboxilatos en la pared del nanocanal. Es decir, ocurre sobrecompensación de la carga en la pared, siendo este fenómeno conocido en la literatura. ${ }^{1} \mathrm{Si}$ $\sigma_{\text {eff }}<0$ entonces ocurre una sub-compensación de la carga en la pared.

$\mathrm{Al}$ disminuir la concentración de sal en solución decrece el efecto de la curvatura sobre la adsorción de polímeros, ver figuras 4.2.1A , 4.2.1B y 4.2.1C. Si bien las cargas en la pared del nanocanal pueden ser apantalladas con los iones proveniente de la sal o con las cadenas de polielectrolitos, al disminuir la concentración de sal también ocurre una disminución de la adsorción, observar el cambio en las escalas de $\sigma_{\text {eff }}$ con la concentración de sal.

Resulta interesante investigar el caso de la figura 4.2.1C, donde la concentración de sal es muy baja $C_{\text {salt }}=10^{-4} M$. En estas condiciones los desbalances en la densidad de carga sobre la superficie son pequeños $\left(\sim 10^{-4}\right)$ y el apantallamiento ocurre exclusivamente por la adsorción de polielectrolitos. Cuando la concentración de polielectrolito es alta, $C_{p o l} \geq 1 \mathrm{mM}$, se observa una sobre compensación de las cargas de la pared $\left(\sigma_{e f f}>0\right)$ y diámetros menores implican

\footnotetext{
${ }^{1}$ Debe su causa al potencial químico de las cadenas poliméricas que debe mantenerse constante (condición de equilibrio) entre su valor para las cadenas adsorbidas y su valor para las cadenas en el seno de la solución. Esto es, hay que tener en cuenta la interacción pared-cadena. Aunque nuestra investigación consideró este caso, aquí mostramos una sobrecompensanción de la carga debido únicamente a interacciones electrostáticas más entropías traslacionales y repulsiones de Van der Waals.
} 


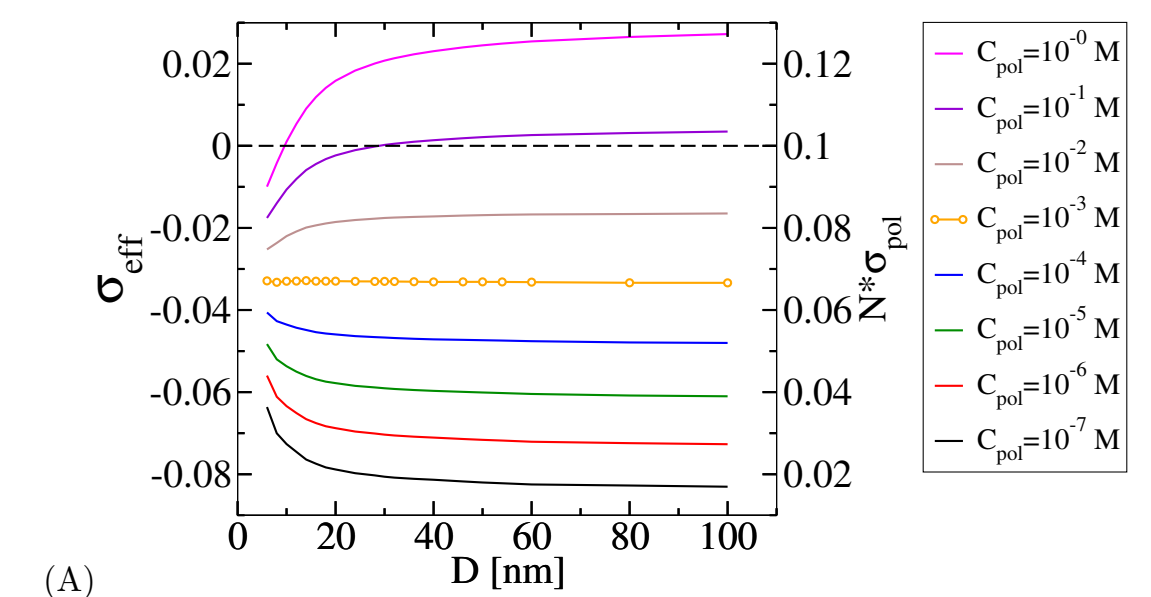

(A)

(B)

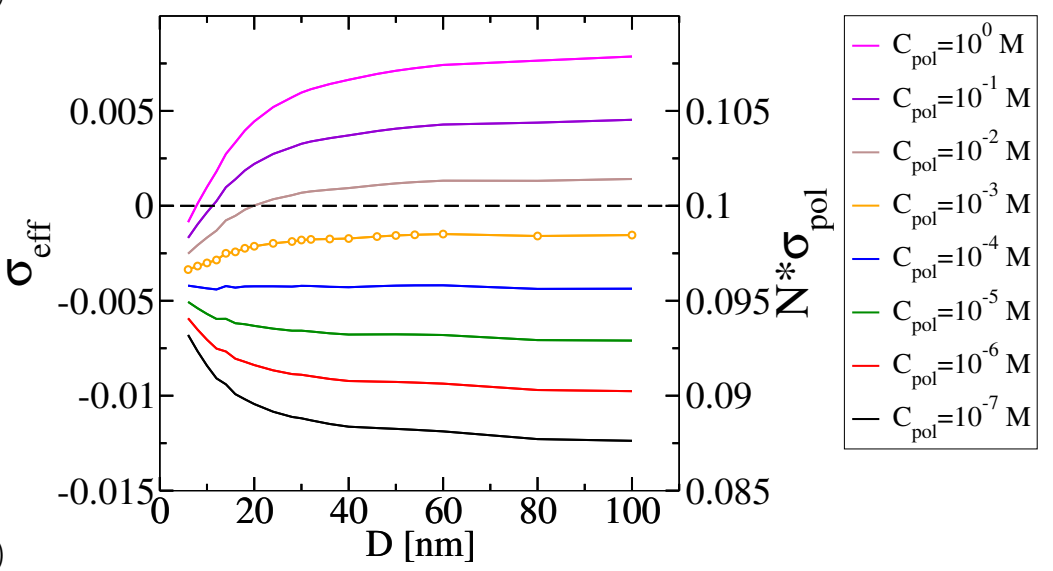

(C)

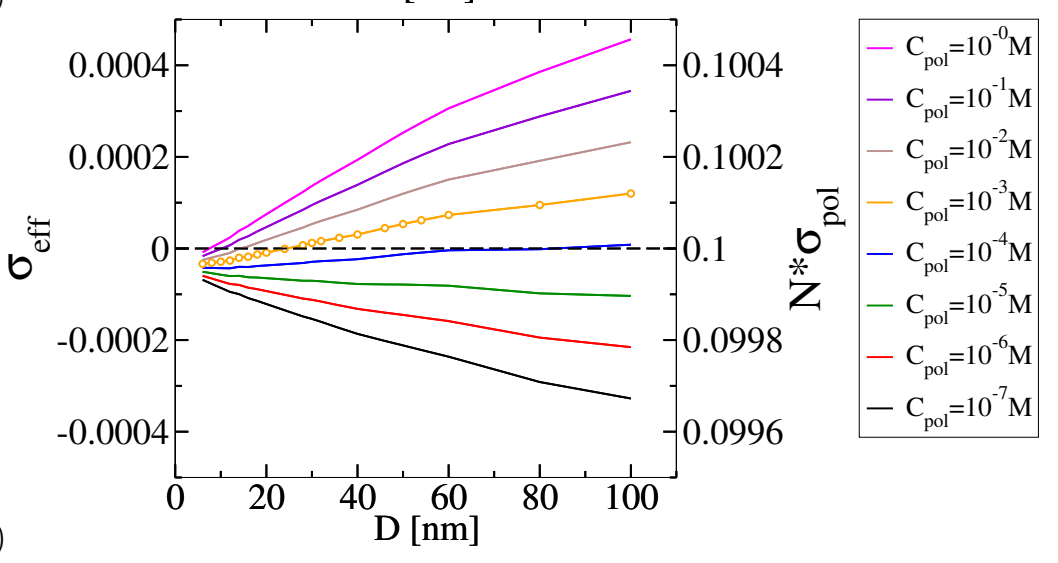

Figura 4.2.1: Densidad de carga efectiva en la superficie del nanocanal, definida como $\sigma_{\text {eff }}=$ $N \sigma_{\text {pol }} f_{1}-\sigma_{\text {wall }}$ en función del diámetro del nanocanal. Las curvas pertenecen a distintos valores de la concentración de polímero en solución, desde $C_{p o l}=10^{-7} M$ hasta $C_{p o l}=1 M$. (A) $C_{\text {salt }}=$ $10^{-1} M$, (B) $C_{\text {salt }}=10^{-2} M$ y (C) $C_{\text {salt }}=10^{-4} M$. El eje de la derecha muestra la densidad de polímero adsorbido en la superficie. Otros parámetros: $N=50, \sigma_{q}=0,1$ 


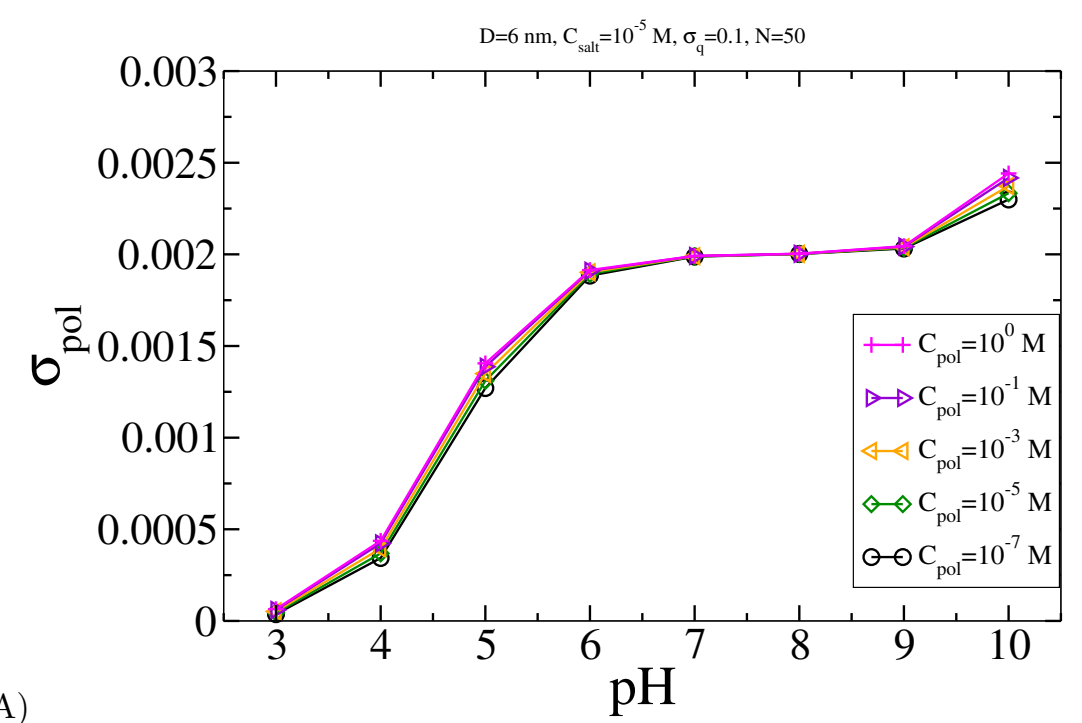

(A)

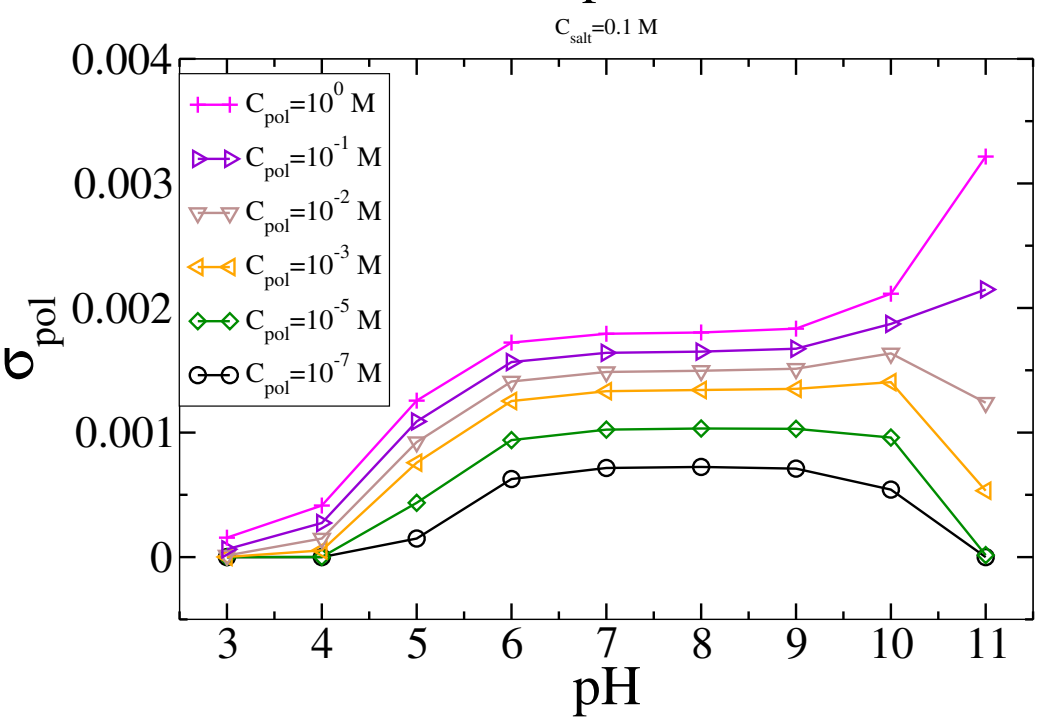

(B)

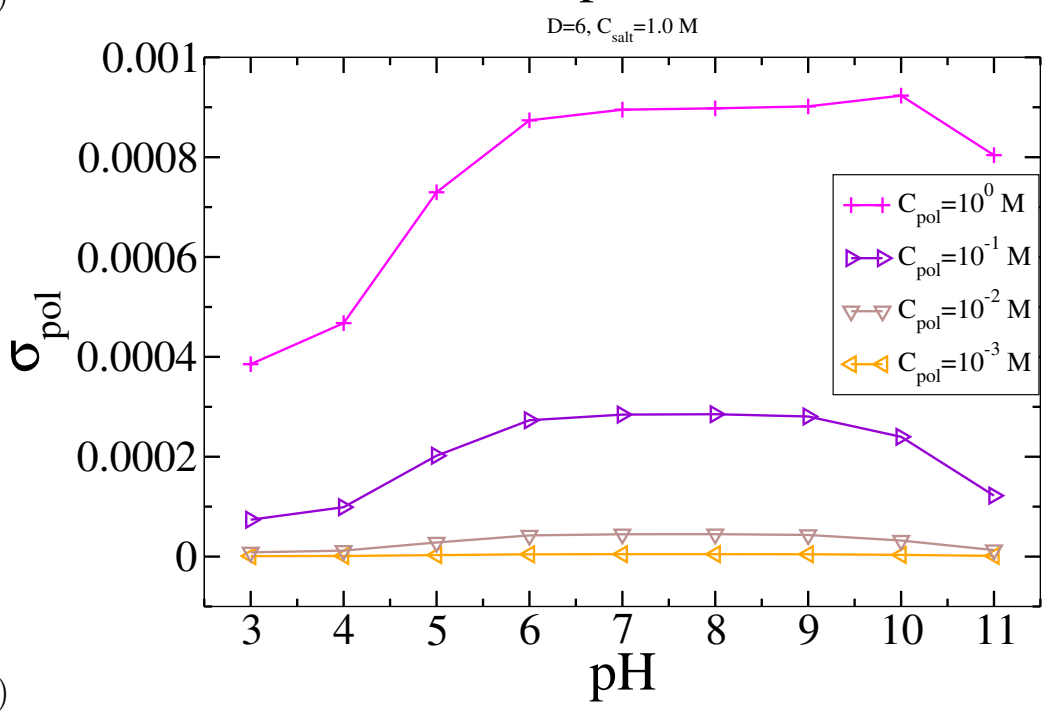

Figura 4.2.2: Densidad superficial de polímero (número de cadenas por $\mathrm{nm}^{2}$ ) en función del $\mathrm{pH}$ de la solución. La concentración de polímero en solución fija el potencial químico del polímero. (A) $C_{\text {salt }}=10^{-5} \mathrm{M}$, (B) $C_{\text {salt }}=0,1 \mathrm{M}$, (C) $C_{\text {salt }}=1,0 \mathrm{M}$. Otros parámetros: $D=6 \mathrm{~nm}$, $\sigma_{q}=0,1 e / n m^{2}, N=50$. 


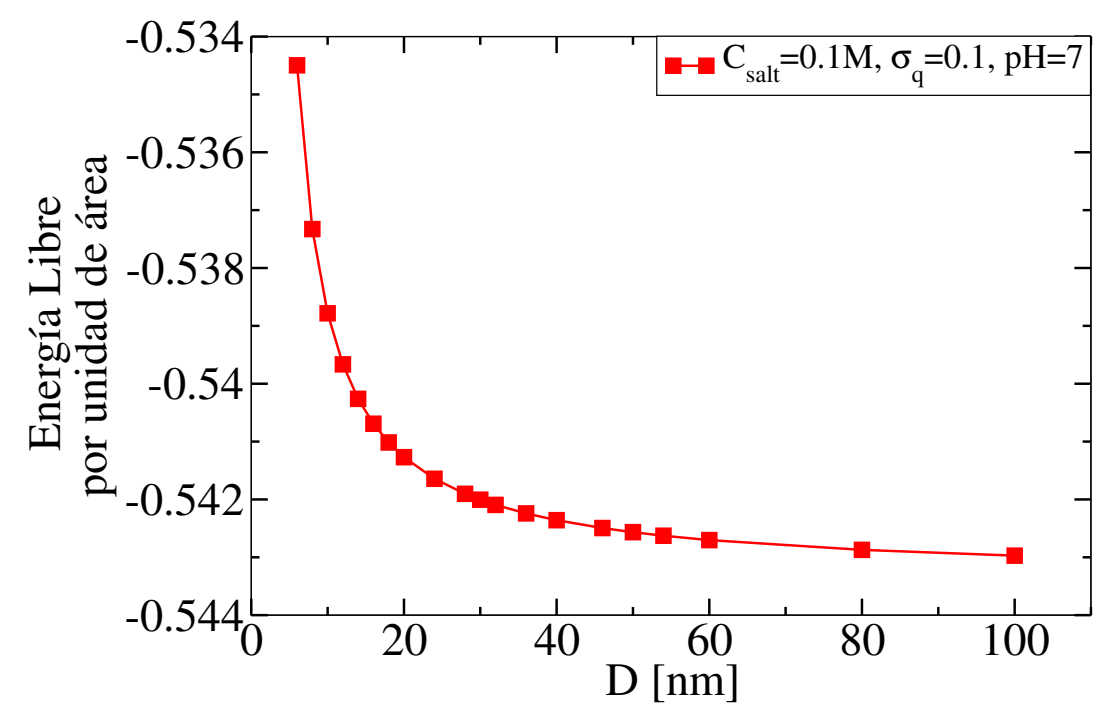

Figura 4.2.3: Energía libre de la superficie en función del diámetro del nanocanal. El sistema tiene carga superficial pero no hay polielectrolitos adsorbidos. Parámetros: $C_{\text {salt }}=0,1 M, \sigma_{q}=$ $0,1 e / n m^{2}, p H=7,0$

una disminución en la cantidad de polielectrolitos adsorbidos. Cuando la carga de la pared esta sub-compensada $\left(\sigma_{e f f}<0\right)$, condición que ocurre si la concentración de polielectrolito es baja $\left(C_{\text {pol }}<10^{-4} M\right)$, entonces diámetros menores implican un aumento en la cantidad de polímero adsorbido. Finalmente en los tres gráficos de la figura 4.2.1, existe una concentración de polímero intermedia para la cual la cantidad de polímero adsorbido es independiente del diámetro del nanocanal.

Para explicar estos resultados consideremos primero un nanocanal sin polímero con densidad de carga superficial constante. A medida que disminuye el diámetro del nanocanal, aumenta la energía libre por unidad de área (ver figura 4.2.3)debido a que la distancia media entre las cargas sobre la pared del nanocanal decrece. Al incorporar polielectrolitos pueden ocurrir dos cosas: si la carga en la pared esta sub-compensada por la carga proveniente de los polielectrolitos entonces, al disminuir el diámetro el sistema compensa el aumento de las repulsiones electrostáticas aumentando la cantidad de polielectrolito adsorbido, de forma tal de disminuir la magnitud de la carga superficial efectiva. Por otro lado, si hay una sobre compensación de la carga superficial entonces, la carga superficial efectiva sobre la superficie es positiva. Este caso es análogo al anterior, cuanto más se reduce el diámetro del nanocanal la cantidad polielectrolito adsorbido disminuye, con el fin de disminuir las repulsiones electrostáticas debido al exceso de carga.

El ejemplo en la figura 4.2.1C discutido en el párrafo anterior es útil para entender el mecanismo a través del cual el nanoconfinamiento regula la cantidad de polielectrolitos adsorbidos. Sin embargo, como dijimos la magnitud del efecto es pequeña. Consideramos que la magnitud del efecto es pequeña porque para concentraciones de sal bajas, el único mecanismo para compensar la carga en la superficie es la adsorción de polielectrolito y, por lo tanto, no puede desviarse demasiado de la condición de compensación absoluta de carga $\sigma_{E f f} \sim 0$ sin aumentar significativamente la energía libre del sistema. En el caso de soluciones de sal intermedias $\left(C_{\text {salt }}=0,1 M\right.$ para la figura $4.2 .1 \mathrm{~A}$ y $0,01 M$ para la figura $\left.4.2 .1 \mathrm{~B}\right)$, los cationes de la sal compiten con el polielectrolito adsorbido para apantallar la carga de la pared. Esto realza el efecto del confinamiento sobre la adsorción de polielectrolitos. Como la carga en la pared esta parcialmente compensada por los cationes de la sal, la densidad de carga efectiva (carga de la pared más carga de los polielectrolitos adsorbidos) para la cuál no hay cambios en la cantidad 
de polielectrolito adsorbido en función de la curvatura del nanocanal, no es $\sigma_{\text {eff }}=0$ sino que corresponde a $\sigma_{\text {eff }}<0$.

También es interesante observar que el efecto del diámetro en la cantidad de polielectrolito adsorbido decae hacia diámetros grandes independientemente del signo de $\sigma_{\text {eff }}$. Este decaimiento es menos pronunciado para concentraciones de sal bajas que para las concentraciones altas y es más grande que $100 \mathrm{~nm}$ para $C_{\text {salt }}=10^{-4} \mathrm{M}$ (figura 4.2.1C). En la práctica esto significa que el efecto de la curvatura en la adsorción de polielectrolitos será importante para concentraciones de sal intermedias, por que para concentraciones de sal bajas el efecto es menor y para concentraciones de sal altas la curvatura afectará sólo los nanocanales angostos.

En la figura 4.2.2 mostramos la densidad superficial de cadenas de polielectrolitos adsorbidos en función del pH de la solución. Cuando $3<p H<9$, aumentar el pH de la solución incrementa la densidad superficial de cadenas adsorbidas por que la pared incrementa su carga según el equilibrio ácido-base de sus grupos carboxilatos, mientras que la carga del polielectrolito se mantiene constante. La densidad superficial de polímero muestra un plateau para valores de $\mathrm{pH}$ mayores a 6 y menores que 9. Esto está en completo acuerdo con la figura 4.0.1C donde se muestra que en esta región de $\mathrm{pH}$ ambos grados de disociación, la disociación de la pared y de los polielectrolitos, se mantienen constantes.

Para valores de $p H>9$ el grado de disociación del polielectrolito disminuye mientras la densidad de carga en la pared se mantiene constante. Este comportamiento depende de la concentración de sal $C_{\text {salt }}$ y la concentración de monómeros $C_{p o l}$ en solución. Cuando la concentración de sal es suficientemente baja $\left(C_{\text {salt }}=10^{-5} \mathrm{M}\right)$, figura $4.2 .2 \mathrm{~A}$, las cargas en la pared están principalmente compensadas por las cargas del polielectrolito. La densidad superficial de cadenas aumenta con el $\mathrm{pH}$ de la solución debido al incremento en la carga de la pared y el comportamiento es independiente de $C_{p o l}$, ver figura 4.2.2A. Para $C_{\text {salt }}=0,1 M$, figura 4.2.2B, hay una competencia entre los iones de la sal y las cadenas de polielectrolitos para apantallar el exceso de la carga en la pared. El sistema intenta minimizar su energía libre y para hacerlo existe un balance entre la entropía traslacional de las cadenas libres y los iones. Para concentraciones $C_{p o l}$ altas la adsorción de cadenas poliméricas es energeticamente favorable por eso hay un incremento en la adsorción de polímero. Al disminuir $C_{p o l}$ se reduce el potencial químico de las cadenas de polielectrolitos, lo que genera un desbalance energético entre iones libres y el polielectrolito, haciendo que sea preferible la adsorción de iones para alcanzar la condición de electroneutralidad.

Cuando $C_{\text {salt }}$ es $1,0 M$, figura $4.2 .2 \mathrm{C}$ la densidad superficial de cadenas $\sigma_{p o l}$, depende de la concentración de polímero $C_{p o l}$ en solución, aunque la adsorción de las cadenas es menor al caso $C_{\text {salt }}=0,1 M$ en la figura 4.2.2B. Si $C_{p o l} \leq 10^{-2} M$, entonces la condición de electroneutralidad en las cercanías de la superficie cargada se cumple principalmente con los iones de la sal $\mathrm{Cl}^{-} \mathrm{y}$ $K^{+}$.

\section{Distribución de polímero dentro del canal}

La distribución radial de los monómeros de las cadenas adsorbidas se muestra en la figura 4.2.4 a una concentración de polielectrolitos en solución $C_{p o l}=1,0 M$ y un $p H=7$ en estas condiciones las cadenas están completamente cargadas y tienen conformaciones tipo varilla (rod-like). Sin embargo, la organización molecular de las cadenas adsorbidas varía con la concentración de sal. Por ejemplo, la capa polimérica sobre la pared del nanocanal se "hincha" cuando aumenta la concentración de sal. Al aumentar $C_{\text {salt }}$ el apantallamiento iónico de la carga polimérica permite conformaciones más flexibles, lo que significa un incremento en la entropía 


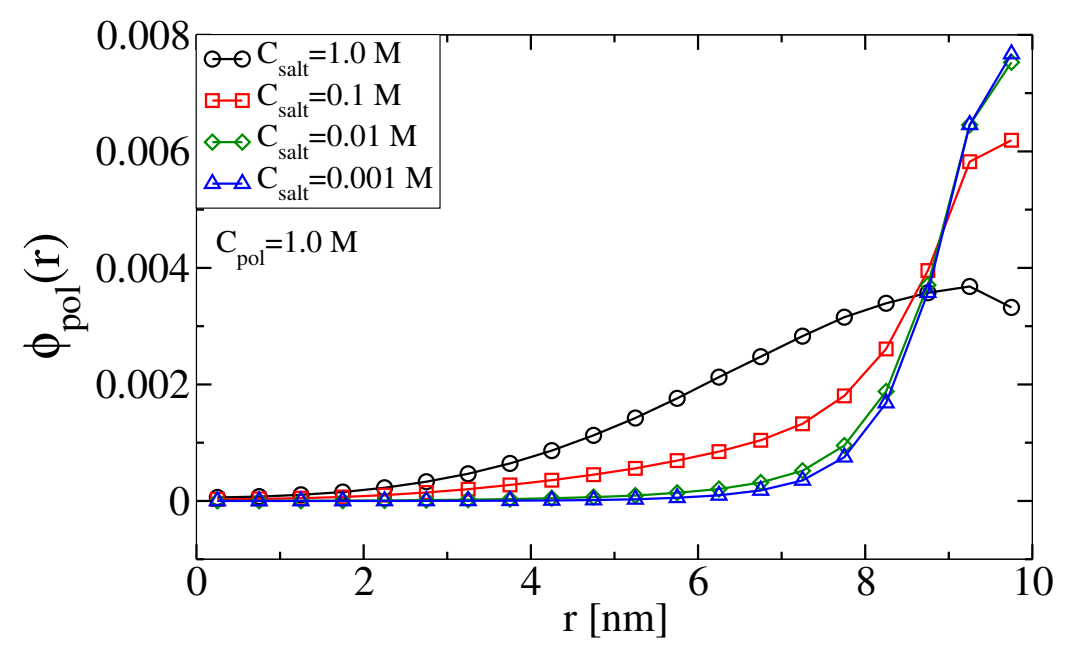

Figura 4.2.4: Fracción de volumen ocupada por el polímero en función de la distancia al centro del nanocanal. Las curvas corresponden a los valores de concentración de sal $C_{\text {salt }}=1,0 \mathrm{M}$, $C_{\text {salt }}=0,1 M, C_{\text {salt }}=0,01 M$ y $C_{\text {salt }}=0,001 M$. Otros parámetros: $\sigma_{q}=0,1 \mathrm{e} / \mathrm{nm}^{2}, N=50$, $C_{\text {pol }}=1,0 M$.

conformacional (capa polimérica más ancha).

En la figura 4.2.5 mostramos la distribución del potencial electrostático dentro del nanocanal, en función de la coordenada radial (distancia al centro del nanocanal) para canales con diámetros $D=10 \mathrm{~nm}$ y $D=100 \mathrm{~nm}$, y concentraciones de sal en solución $C_{\text {salt }}=10^{-5} \mathrm{M}$ y $C_{\text {salt }}=1 M$. Si comparamos la figura 4.2.5A2 con la figura 4.2.5B2, observamos que el potencial electrostático $\Psi(r)$ decrece más rápido cuando $C_{\text {salt }}$ es mayor, esto es por que la concentración de sal determina la longitud característica de las interacciones electrostáticas (longitud de Debye $\lambda_{D}=\left(\frac{\varepsilon k_{B} T}{\sum_{j=1}^{N} n_{j} q_{j}^{2}}\right)^{1 / 2}$ ). Por otro lado el diámetro del nanocanal determina el grado de confinamiento, haciendo que los iones libres provenientes de la sal deban interactuar con la doble capa eléctrica cerca de la pared del nanocanal. El valor del potencial electrostático en el centro del nanocanal está determinado por $C_{\text {salt }}$ y $D$. Para diámetros chicos y concentraciones de sal bajas, ver figura 4.2.5A1, el potencial puede considerarse constante si la distancia a la pared del nanocanal es mayor a $1 \mathrm{~nm}$.

Consideremos a continuación un nanocanal largo no cilíndrico. donde $D \ll L$. En este caso las propiedades del nanocanal a una dada posición $z$ sobre el eje principal, pueden ser aproximadas localmente por las propiedades de un nanocanal cilíndrico de diámetro $D(z)$, donde $D(z)$ es el diámetro de un nanocanal no cilíndrico dependiente de la posición $z[25]$. De acuerdo con nuestros resultados, la cantidad de polielectrolito adsorbido y el perfil del potencial electrostático dentro del nanocanal dependen del diámetro, por eso esperamos que en el equilibrio la distribución de polielectrolitos adsorbidos en la superficie interna de nanocanales no cilíndricos inducirá una carga efectiva $\sigma_{\text {eff }}$ inhomogenea sobre la pared del nanocanal.

Los nanocanales asimétricos con densidad de carga homogénea o nanocanales cilíndricos con distribuciones de carga homogéneas pueden rectificar la corriente iónica. Nos preguntamos ahora como cambiarán las propiedades de transporte de un canal no cilíndrico con una adsorción inhomogenea de polielectrolitos. Utilizamos el software COMSOL para resolver las ecuaciones de Poisson-Nernst-Planck sin aproximaciones a través del cálculo por elementos finitos $(E F)$. Mostramos los resultados en la figura 4.2.6, las curvas $I-V$ (corriente-voltaje) que se predicen para un nanocanal cónico con una densidad de carga neta $\sigma_{e f f}$, determinada por las curvas de la figura 4.2.1. Se observa que cuando $C_{p o l}=0,1 M$, si la concentración de sal es $C_{\text {salt }}=$ 
(A1)



(A2)

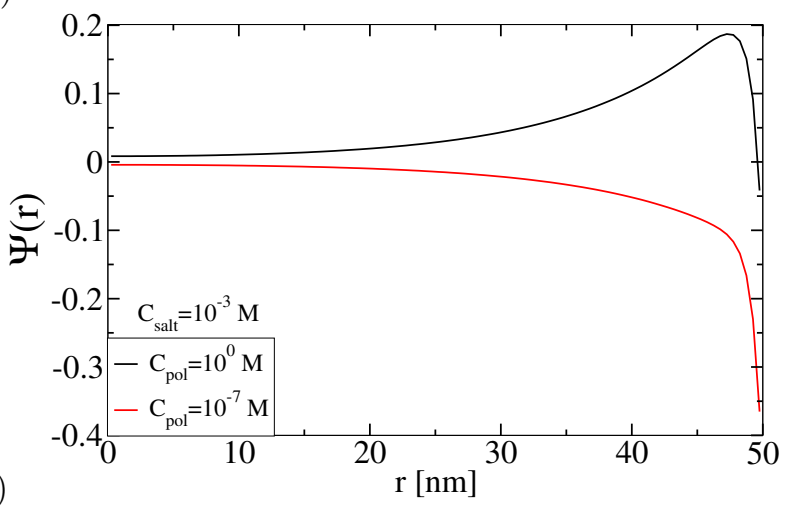

(B1)
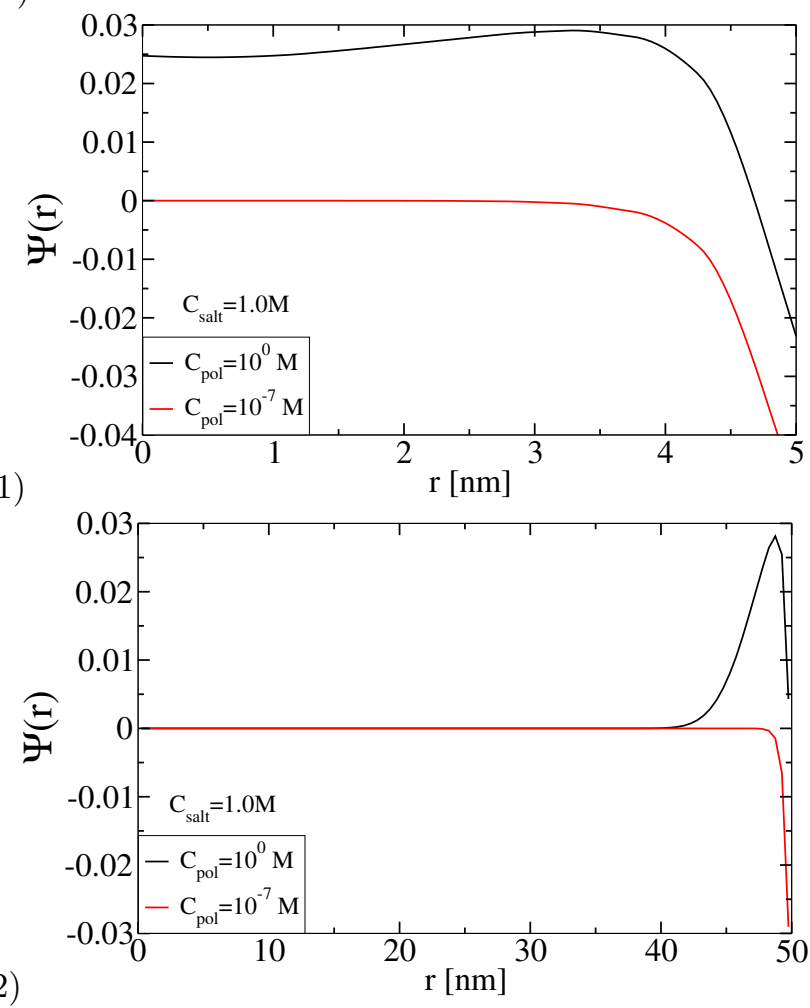

Figura 4.2.5: Potencial electrostático dentro del nanocanal en función de la distancia al centro del nanocanal. (A1) $D=10 \mathrm{~nm}, C_{\text {salt }}=10^{-3} \mathrm{M}$, (A2) $D=100 \mathrm{~nm}, C_{\text {salt }}=10^{-3} \mathrm{M}$, (B1) $D=10 \mathrm{~nm}, C_{\text {salt }}=1,0 M$ y (B2) $D=10 \mathrm{~nm}, C_{\text {salt }}=1,0 M$. Los cambios en el potencial electrostático con la concentración de sal están relacionados con el cambio en la longitud de Debye $\lambda_{D}$. El grado de confinamiento (tamaño del diámetro) modifica el potencial en el centro del canal. 


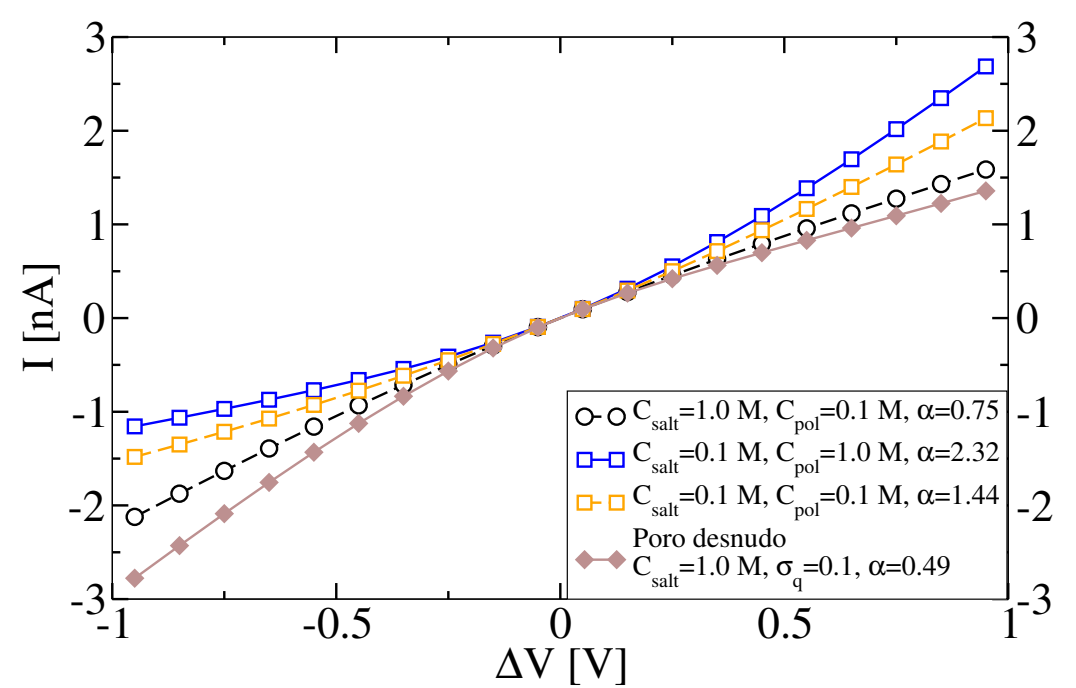

Figura 4.2.6: Corriente iónica en función del voltaje aplicado. Entre los extremos de un nanocanal cónico largo $(L \gg D)$,modificado con una capa de PAH. La distribución de PAH es no uniforme y sigue las predicciones hechas por la teoría molecular.

$1,0 M$ (círculos blancos) la rectificación es en una dirección y si $C_{\text {salt }}=0,1 M$ (cuadrados blancos) se cambia la dirección de la rectificación. Esto evidencia una inversión de carga en la superficie interna del nanocanal. En cambio si $C_{\text {salt }}=0,1 M$ y variamos la concentración de polielectrolitos de $C_{p o l}=0,1 M$ a $C_{p o l}=1,0 M$ (cuadrados obscuros) vemos que aumenta el grado de rectificación desde $\alpha=1,44$ a $\alpha=2,32$. Estos resultados son compatibles con las observaciones experimentales. 


\section{Capítulo 5}

\section{Efectos de Confinamiento en Nanocanales Modificados Con Brushes Polipróticos}

La teoría molecular es una teoría de campo medio que ha logrado describir correctamente nanocanales cilíndricos con regulación de cargas en la superficie interna. A través de ciertas aproximaciones permite relacionar el detalle molecular con alguna propiedad macroscópica dependiente de las características del sistema en equilibrio. En este capítulo se utiliza la teoría molecular extendida a nanocanales largos, no cilíndricos. El objetivo es obtener el comportamiento macroscópico de la conductancia de nanocanales no-cilíndricos y relacionar este comportamiento con la estructura y composición de la capa polimérica anclada en la superficie interna de estos nanocanales largos $(r<<L)$ con geometría arbitraria. Esta metodología se utiliza para explorar el acoplamiento entre el equilibrio ácido-base y la geometría en nanocanales cilíndricos, cónicos y con forma de trompeta, modificados con un brush polimérico de poli(2-(metacriloiloxi) etil-fosfato (PMEP), un ácido diprótico.

Nos enfocamos en el estudio de la conductancia iónica y las curvas de especiación (la fracción de segmentos deprotonados, monoprotonados y diprotonados) como función del $\mathrm{pH}$ de la solución. Veremos que las constantes de equilibrio $(p K a)$ y el ancho de las transiciones entre los distintos estados ácido-base determinados con las curvas de especiación dependen del diámetro, la geometría y la concentración de sal del bulk. Estudiaremos el límite de nanocanales anchos, allí las constantes de equilibrio y los anchos de las transiciones pueden estimarse con un modelo analítico derivado de la teoría más general presentada en los primeros capítulos.

Tanto la teoría molecular como el modelo simplificado predicen que debido a efectos de regulación de carga la primera transición ácido-base transición (0/-1) es más ancha que la segunda transición (-1/-2), ambas transiciones son más anchas que la transición ideal esperada para un grupo ácido-base aislado en bulk. Se mostrará también que el punto de inflexión que aparecen en las curvas de conductancia proveen una buena estimación de las constantes de equilibrio aparente $p K a$ de polielectrolitos en canales cilíndricos pero que la calidad de la estimación disminuye en geometrías no-cilíndricas. 




Figura 5.0.1: Esquema de una sección del nanocanal modificado con un poliácido débil (poli(2(metacriloiloxi)etil-fosfato), PMEP, anclado a la pared interna del nanocanal. La solución dentro del nanocanal contiene moléculas de agua, cationes (círculos azules) y aniones (círculos rojos) provenientes de la sal $\mathrm{KCl}$, aniones $\mathrm{OH}^{-}$y cationes $\mathrm{H}_{3} \mathrm{O}^{+}$. Cada monómero de PMEP tiene tres estados químicos posibles: $\mathrm{PMEPH}_{2}\left(\mathrm{AH}_{2}\right), \mathrm{PMEPH}^{-}\left(A H^{-}\right), P M E \mathrm{P}^{2-}\left(A^{2-}\right)$.

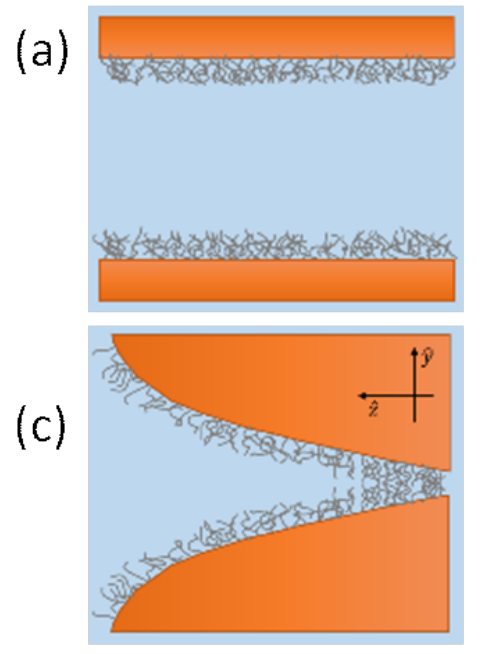

(b)

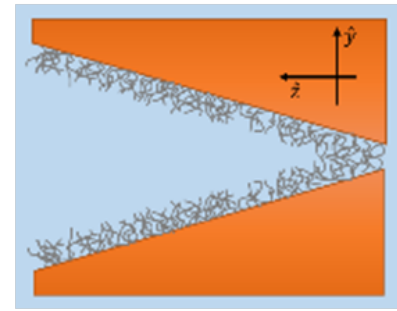

(d)

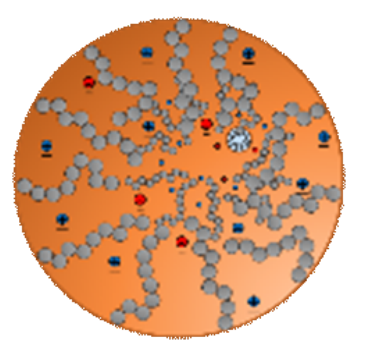

Figura 5.0.2: Los paneles (a), (b), y (c) muestran esquemas con las geometrías exploradas. Estos esquemas no están en escala, recordar que $L=12 \mu m$ y $D \leq 600 \mathrm{~nm}$. Localmente cada geometría puede ser aproximada por cilindros debido a la relación de aspecto del nanocanal. El panel (d) muestra una vista transversal del nanocanal. Todas las geometrías exploradas tienen simetría cilíndrica, es decir, las inhomogeneidades se encuentran únicamente en la dirección radial $\hat{r}$ y en la dirección del eje principal del nanocanal $\hat{z}$. 


\subsection{Conductancia de Nanocanales con Geometrías Cilín- dricas y No-Cilíndricas}

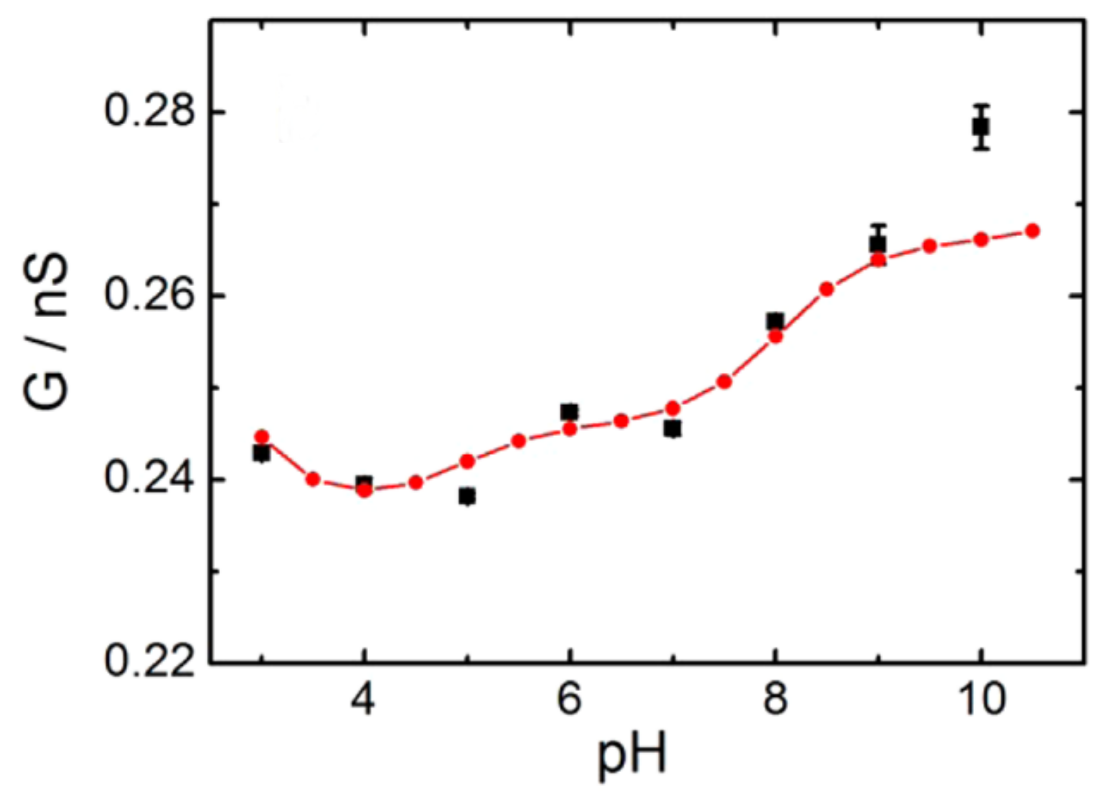

Figura 5.1.1: Con símbolo y barras de error curva experimental de conductancia en función del pH, para un nanocanal modificado con PMEP, en línea llena se muestra el ajuste teórico. La conductancia experimental se obtuvo de las curvas de corriente $I$ en función del voltaje $V$ cuando $V \rightarrow 0$ para el nanocanal modificado con PMEP (datos publicado en la figura 3a de la referencia [80]; las barras de error representan el error asociado a la estimación de la pendiente de las curvas alrededor de $V=0$ ).

El modelo desarrollado anteriormente nos permite calcular la conductancia en condiciones de cuasi-equilibrio (low-bias) de poros largos modificados con polielectrolitos con geometría arbitraria. La validación de nuestro modelo se realizó comparando los resultados en dos escenarios distintos. El primer escenario consiste en un nanocanal cónico con densidad de carga superficial constante ( $\sin$ la presencia de polielectrolitos adheridos a la superficie). Elegimos este sistema por que puede ser descripto con las ecuaciones de Poisson-Nernst-Planck y resuelto sin aproximaciones a través del cálculo por elementos finitos $(E F)$. El cálculo por elemento finitos es una técnica que consiste en discretizar las ecuaciones diferenciales que describen el sistema y luego resolver numéricamente. Existe software privativo que trae implementado esta técnica. Para la comparación con nuestro modelo, utilizamos el software Comsol. La figura 5.1.1(a) muestra que la conductancia predicha por el modelo de particiones está en completo acuerdo con los resultados obtenidos por el método de $E F$ que considera explícitamente el nanocanal completo.

En el segundo escenario se comparó los resultados de la teoría con resultados experimentales. El sistema experimental reportado [80] consiste en un nanocanal de geometría no cilíndrica, modificado con PMEP. Experimentalmente se logra determinar algunas características geométricas como pueden ser el largo del nanocanal y el diámetro de la base. Esta información se incorpora a nuestro modelo para poder comparar el comportamiento macroscópico medido con el predicho por la teoría. Los puntos negros en la figura 5.1.1(b), representan las conductancias experimentales determinadas como la pendiente de las curvas $I-V$, reportadas en la figura 3(a) de la referencia [80], en el límite $V \rightarrow 0$. Para aproximar los datos experimentales utilizamos las dimensiones del nanocanal reportadas[80]: $D_{\min }=16 \mathrm{~nm}, D_{\max }=290 \mathrm{~nm}, \mathrm{~L}=12 \mu \mathrm{m}$, las cons- 
tantes de equilibrio de bulk del PMEP y la concentración de sal de la solución $C_{\text {salt }}=0,1 M$. Permitimos que el grado de polimerización $N$ y el recubrimiento superficial $\sigma$ variaran (usamos $N=14$ y $\sigma=0,01$ cadenas $/ \mathrm{nm}^{2}$; en la práctica el parámetro relevante es $N \times \sigma[66]$, por lo que otras combinaciones de $N$ y $\sigma$ son posibles). También permitimos que la geometría se aparte levemente del cono truncado ideal. Utilizamos $n=1,3$ en 3.9, que genera una geometría cercana al cono $(n=1)$. Podemos decir que este modelo teórico está en completo acuerdo con los cálculos realizados por EF 5.1.1(a), en donde la comparación se realiza con la solución numérica exacta del sistema cónico, y describe correctamente los resultados experimentales 5.1.1(b), por lo tanto, queda validada su utilización para el estudio de la conductancia de nanocanales cilíndricos y no cilíndricos, largos. El comportamiento macroscópico del sistema surge naturalmente de sus propiedades microscópicas. ¿Cómo es la organización molecular compatible con los comportamientos macroscópicos que podemos medir? ¿Son éstas propiedades indiferentes al confinamiento? A continuación describimos la organzación microscópica del sistema con la lupa que nos brinda la teoría.

\subsection{Equilibrios Ácido-base y Conductancia en Nanocana- les Cilíndricos}

El marco teórico que utilizamos nos brinda una descripción microscópica compatible con el comportamiento macroscópico del sistema. A continuación se muestra la descripción microscópica que se obtiene como resultado de la minimización de la energía libre que describe al sistema.

La figura 5.2.1 muestra las predicciones para un nanocanal cilíndrico modificado con un polielectrolito poli(2-(metacriloiloxi)etil-fosfato. El diámetro del nanocanal es $D=16 \mathrm{~nm}$ y la concentración de sal $C_{\text {salt }}=0,1 M$. La Figura 5.2.1a muestra la dependencia del grado de disociación $\left\langle f_{e}\right\rangle$ con el $\mathrm{pH}$ de la solución. El subíndice $e=0,-1,-2$ nombra cada uno de los tres estados de carga accesibles al monómero de PMEP (ver figura 5.0.1). Estos estados de carga se corresponden con las especies del ácido fosfórico: neutra $\left(\mathrm{AH}_{2}=\mathrm{R}-\mathrm{PO}_{4} \mathrm{H}_{2}, e=0\right)$, cargada monovalentemente $\left(A H^{-}=R-P_{4} H^{-}, e=-1\right)$ y el fosfato cargado divalentemente $\left(A^{2-}=R-P O_{4}^{2-}, e=-2\right)$. La transición entre estados de carga se caracteriza por una constante de equilibrio, para el fosfato (grupo protonable del monómero de PMEP) las constantes de equilibrio químico son $p K_{a 1}^{b u l k}=4,5$ y $p K_{a 2}^{b u l k}=7,7$. Estos valores fueron medidos en solución y por lo tanto no consideran los efectos de confinamiento sobre el equilibrio ácido-base.

La teoría desarrollada considera el detalle molecular de las especies involucradas y realiza una descripción microscópica local, por lo tanto incorpora los efectos locales del entorno sobre la transición entre estados. Esta descripción teórica da lugar a constantes de equilibrio aparentes dentro del nanocanal cuyos valores resultan distintos a los valores de bulk. A continuación discutiremos no sólo el corrimiento de estos valores respecto de los valores de bulk, sino que también mostramos cómo se modifica el ancho de estas transiciones.

El primer equilibrio químico involucra la transición entre el estado neutro (a $\mathrm{pH}=2.0$ ) y el estado con carga -1 (a $p H \approx 8,0$ ). En este rango de $\mathrm{pH}$ podemos considerar que el número de monómeros con carga -2 es constante y muy cercano a cero. Para valores de pH mayores a 8 , se inicia la segunda transición, en donde la fracción de monómeros en el estado neutro es cercano a cero $\left(\left\langle f_{0}\right\rangle \approx 0\right)$ y el número de monómero en el estado - 2 se incrementa a expensas de la población de monómeros en el estado -1 . El incremento de la carga polimérica ocurre hasta $p H=12$ aproximadamente. Para valores de $\mathrm{pH}$ mayores, las cadenas de PMEP se comportan como 

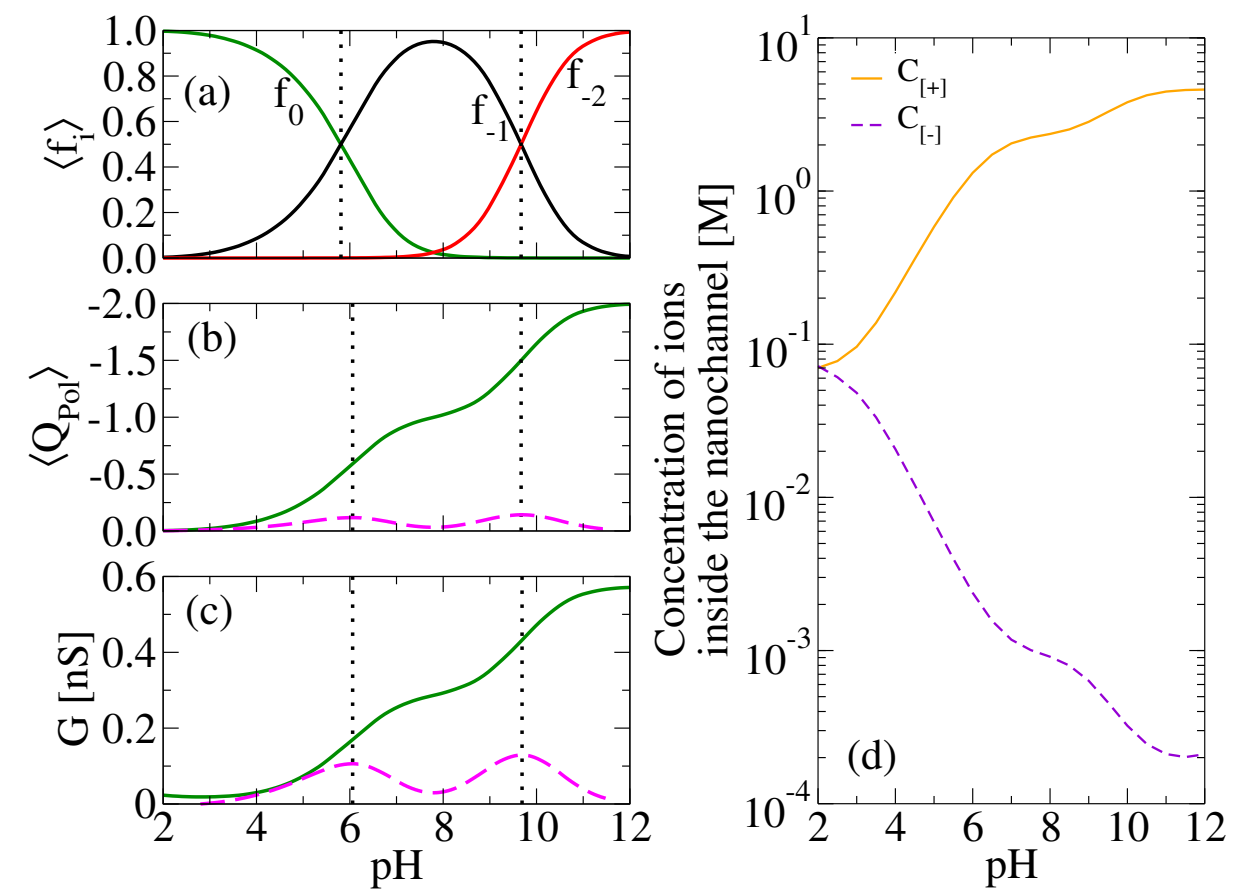

Figura 5.2.1: Comportamiento de un nanocanal cilíndrico modificado con PMEP en la superficie interna. $p K a_{1}^{\text {bulk }}=4,5, p K a_{2}^{\text {bulk }}=7,7$. (a) Grado de disociación medio. $\left\langle f_{0}\right\rangle$ fracción de monómeros en el estado neutro, $\left\langle f_{-1}\right\rangle$ fracción de monómero en el estado con carga $-1, \mathrm{y}$ fracción de monómero en el estado con carga $-2,\left\langle f_{-2}\right\rangle$, son mostradas en verde, negro y rojo respectivamente. El pKa aparente $\left(p K a^{a p p}\right)$ fue determinado desde las curvas de $\mathrm{pHs}$ donde $\left\langle f_{0}\right\rangle=\left\langle f_{-1}\right\rangle\left(p K a_{1}^{a p p}=5,81\right)$ y $\left\langle f_{-1}\right\rangle=\left\langle f_{-2}\right\rangle\left(p K a_{1}^{a p p}=9,67\right)$. (b) Carga media por monómero en función del pH de la solución (línea rayada en magenta). (c) Conductancia del canal (línea sólida en verde) y su primera derivada con respecto al $\mathrm{pH}$ (línea rayada en magenta). Los pKa aparentes estimados a partir del máximo de la primer derivada son: $p K a_{1}^{\text {cond }}=6,06 \mathrm{y}$ $p K a_{1}^{\text {cond }}=9,69$. (d) Concentración media de aniones y cationes dentro del nanocanal en función del $\mathrm{pH}$ de la solución. Parámetros utilizados: $D=16 \mathrm{~nm}, C_{\text {salt }}=0,1 \mathrm{M}, \mathrm{N}=28$ unidades por cadena, $N_{P} / A(R)=0,2^{\text {cadenas }} / \mathrm{nm}^{2}$. 

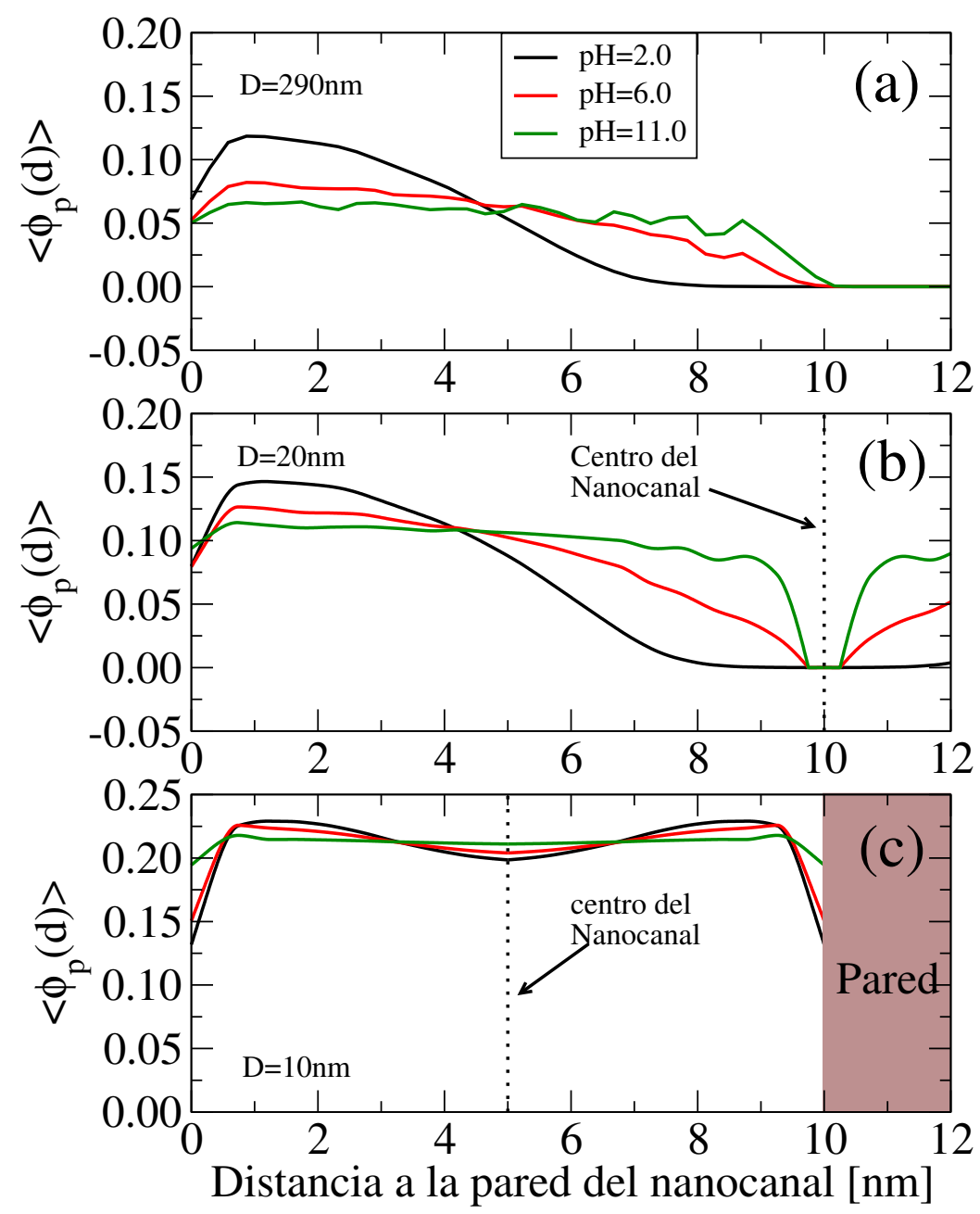

Figura 5.2.2: Fracción de volumen del polímero $\left\langle\phi_{P}(d)\right\rangle$ en función de la distancia a la pared del nanocanal, $d$. Las curvas corresponden a distintos valores del $\mathrm{pH}$ de bulk: 2.0 (negro), 6.0 (rojo), 11.0 (verde). Los diámetros de los nanocanales son: (a) $D=290 \mathrm{~nm}$, (b) $D=20 \mathrm{~nm}$, (c) $D=10 \mathrm{~nm}$. Otros parámetros: $N=28$ unidades por cadena, $N_{P} / A(R)=0,2$ cadenas $/ \mathrm{nm}^{2}$, $C_{\text {salt }}=0,1 M$. 
un polielectrolito fuerte donde todos los grupos están completamente deprotonados. Interesa conocer el comportamiento de la carga promedio por monómero $\left\langle Q_{m o n}\right\rangle=-\left\langle f_{1}\right\rangle|e|-2\left\langle f_{2}\right\rangle|e|$ porque el exceso de carga fija confinada dentro del nanocanal es el responsable del incremento de la conductancia del nanocanal. Se muestra en la figura 5.2.1(b) como $\left\langle Q_{m o n}\right\rangle$ incrementa su valor absoluto a medida que aumenta el valor de $\mathrm{pH}$ y alcanza su máximo $\left\langle Q_{\text {mon }}\right\rangle=-2|e|$ cuando $p H \approx 12$. La conductancia del sistema $(G)$ en 5.2.1(c) también aumenta al aumentar el $\mathrm{pH}$. Este efecto es debido al incremento en la concentración de contra-iones dentro del canal, necesario para compensar la creciente carga del polielectrolito. Estos contra-iones son cargas móviles y por lo tanto aumentan la conductancia del nanocanal de acuerdo con la ecuación 3.4. A $p H=12$ el nanocanal es altamente selectivo, la teoría predice una concentración de contra-iones 4 ordenes de magnitud superior a la concentración de co-iones; ver figura 5.2.1(d). Dada la asimetría en las concentraciones de iones libres dentro del nanocanal se dice que la solución dentro del nanocanal es una solución "unipolar"[18].

Las constantes de equilibrio químico $p K_{a}$ están definidas iguales a los valores de $\mathrm{pH}$ donde la fracción de monómeros de los dos estados involucrados son iguales. Esta es la definición habitual de las constantes aparentes en solución. En confinamiento utilizamos el mismo criterio, definimos la primera y la segunda constante de equilibrio químico aparente como los valores de $\mathrm{pH}$ de la solución para los cuales $\left\langle f_{0}\right\rangle=\left\langle f_{-1}\right\rangle$ y $\left\langle f_{-1}\right\rangle=\left\langle f_{-2}\right\rangle$, respectivamente. Con esta definición la teoría predice que los valores de las constantes de equilibrio aparente $p K_{a}$ de los polielectrolitos confinados son diferentes de los valores de bulk[66]. Por ejemplo, hallamos que $p K_{a 1}^{a p p}=5,81$ y $p K_{a 2}^{a p p}=9,67$ para el sistema de la figura 5.2.1 $(\mathrm{N}=28$ monómeros por cadena, $\left.N_{p} / A(R)=0,2_{\text {cadenas }} / \mathrm{nm}^{2}, D=16 \mathrm{~nm}, C_{\text {salt }}=0,1 M\right)$. Para determinar las constantes de equilibrio aparentes $p K_{a 1}^{a p p}$ es necesario conocer el grado de disociación promedio dentro del nanocanal, el problema que tiene esta magnitud es que no es accesible experimentalmente. Por otro lado la conductancia del nanocanal es una cantidad medible que está relacionada con la cantidad de carga confinada y por lo tanto se la utiliza para estimar el estado de carga del polielectrolito[66]. Para estudiar la exactitud en la estimación de las constantes aparentes $p K_{a}$ a partir de los datos de conductancia, definimos las constantes de equilibrio $p K_{a 1}^{c o n d}$ y $p K_{a 2}^{c o n d}$ como el valor de $\mathrm{pH}$ donde la primer derivada de la conductancia alcanza el máximo, es decir, el punto de inflexión de las curvas $G$ vs $p H$. Usaremos los superíndices "cond" para indicar las constantes de equilibrio estimadas a partir de la conductancia. Las líneas punteadas verticales en la figura 5.2.1(c) muestran las $p K_{a}^{\text {cond }}$ determinadas a partir de las curvas de conductancia predichas. En el caso de nanocanales cilíndricos, figura 5.2.1, las constantes de equilibrio aparentes para ambos equilibrios ácido-base estimados desde las curvas de conductancia son compatibles con los valores obtenidos a partir de los grados de disociación dentro del nanocanal. El valor de $p K_{a 1}^{c o n d}=6,06$ es un poco más grande que $p K_{a 1}^{a p p}=5,81$ debido a una pequeña contribución de contra-iones a la conductancia total, relacionados con la electroneutralidad de la especie $R-P O_{4}^{2-}$, cuando $p H=p K_{a 1}^{a p p}$. Los métodos de estimación de las constantes de equilibrio aparentes son sensibles a la geometría del nanocanal. Veremos más adelante que para nanocanales no cilíndricos, el acuerdo entre las constantes de equilibrio $p K_{a 1}^{\text {cond }}, p K_{a 2}^{\text {cond }}$ y $p K_{a 1}^{a p p}, p K_{a 2}^{a p p}$, empeora ligeramente.

El exceso de carga en las cadenas poliméricas provoca cambios en la organización molecular del sistema. A continuación mostramos cómo se reorganiza la capa polimérica. 


\section{Distribución de polímero}

Las cadenas poliméricas totalmente extendidas tienen una longitud de $h \approx 10 \mathrm{~nm}$, esta longitud es la que debe compararse con el radio del nanocanal para obtener una estimación cualitativa del grado de confinamiento. Por otro lado debemos considerar la longitud de Debye $\lambda_{D}=\sqrt{\frac{\varepsilon k_{B} T}{\sum_{j} n_{j} q_{j}^{2}}}$ que mide el alcance de la interacción electrostática en un medio con cargas móviles. Cuando el radio del canal es mayor o igual a $10 \mathrm{~nm}$ (figura $5.2 .2 \mathrm{a}$ con $D=290 \mathrm{~nm}$ y figura 5.2.2b con $D=20 \mathrm{~nm}$ ), aumentar el $\mathrm{pH}$ de la solución hace que la capa de polielectrolitos se expanda hasta su ancho máximo 10nm. Este cambio conformacional ocurre para disminuir la repulsión electrostática. Las cadenas de polielectrolito adoptan conformaciones más extendidas a medida que se cargan sus unidades monoméricas.

Este acoplamiento entre el estado de carga de los monómeros y las conformaciones de cadena es menos importante en el segundo equilibrio químico. Durante la segunda deprotonación los monómeros ya se encuentran cargados y por lo tanto el polielectrolito está casi completamente extendido. Este resultado muestra que la capacidad de estiramiento del polímero está saturada luego de la primera deprotonación.

Para entender el rol de la geometría queremos comparar el comportamiento de las cadenas confinadas en el nanocanal con un brush polimérico sobre una superficie plana. Esta comparación surge naturalmente si pensamos que para un diámetro suficientemente grande las cadenas interactúan localmente como si estuvieran en una superficie plana. Para cadenas totalmente extendidas en una superficie plana (o un nanocanal suficientemente ancho), la fracción de volumen de polímero $\left\langle\phi_{p}\right\rangle$ puede ser estimada suponiendo que el perfil de densidad es una función escalón con ancho 10nm. Bajo esta aproximación se obtiene la fracción de volumen $\left\langle\phi_{p}\right\rangle \approx N_{p} N v_{p} / A(R) h=0,053$. Este resultado esta en completo acuerdo con lo que se muestra en la figura 5.2.1a para canales suficientemente anchos $(D=290 \mathrm{~nm})$.

En el caso de canales angostos $D=20 \mathrm{~nm}(h \approx D / 2)$ se observa una fracción de volumen $\left\langle\phi_{p}\right\rangle \approx 0,11$ (ver curva verde en 5.2.1b). Vemos que en este caso, la fracción de volumen de polímero es más grande que el valor esperado para superficies planas. Esto se debe a un efecto de curvatura, las superficies curvadas hacen que el volumen disponible para los monómeros disminuya al acercarnos al centro del nanocanal. Por lo tanto, si se mantiene constante el recubrimiento de cadenas poliméricas (número de cadenas por unidad de área), la densidad promedio de monómeros de la capa polimérica aumentará al disminuir $D$.

La figura 5.2.2c muestra la fracción de volumen de polímero dentro del nanocanal con diámetro $D=10 \mathrm{~nm}$ y diferentes valores de $\mathrm{pH}$ de solución. En este caso el radio del canal es menor que la longitud de las cadenas poliméricas extendidas, por lo tanto la distribución de monómeros $\left(\left\langle\phi_{p}\right\rangle\right)$ es homogénea dentro del nanocanal e independiente del estado de carga de los monómeros. En este caso, $\left\langle\phi_{p}\right\rangle \approx 0,2$, la densidad de monómeros es mucho más grande que el valor predicho para superficies planas debido al solapamiento de las capas poliméricas en el centro del canal y efectos de curvatura explicados en el caso anterior.

\section{Efectos de confinamiento en los equilibrios químicos}

En canales cilíndricos con diámetro $D=16 n m$ y concentración de sal $0,1 M$ (ver figura 5.2.1) la teoría predice que las constantes de equilibrio aparente deben tener valores $p K_{a 1}^{a p p}=5,81 \mathrm{y}$ $p K_{a 2}^{a p p}=9,67$, estos valores son más grandes que las correspondientes a los equilibrios químicos en solución: $p K_{a 1}^{b u l k}=4,5$ y $p K_{a 2}^{b u l k}=7,7$. Este desplazamiento de 1.3 y 2.0 unidades de pH, emerge de la competencia entre las interacciones y la organización molecular para minimizar la energía libre del sistema. A medida que aumenta el pH de la solución la carga neta de los 


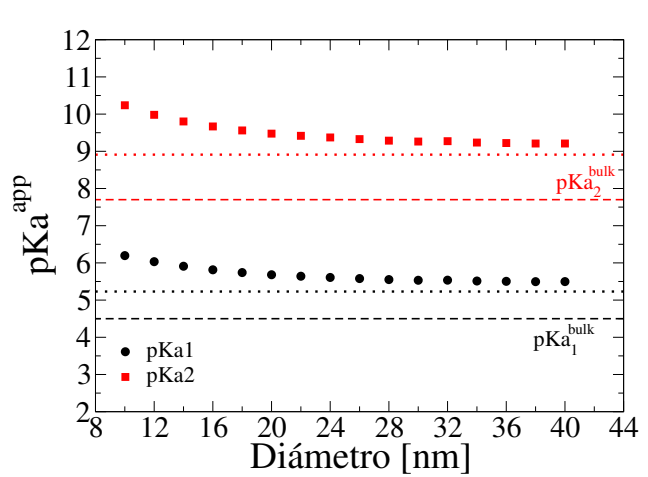

(a)

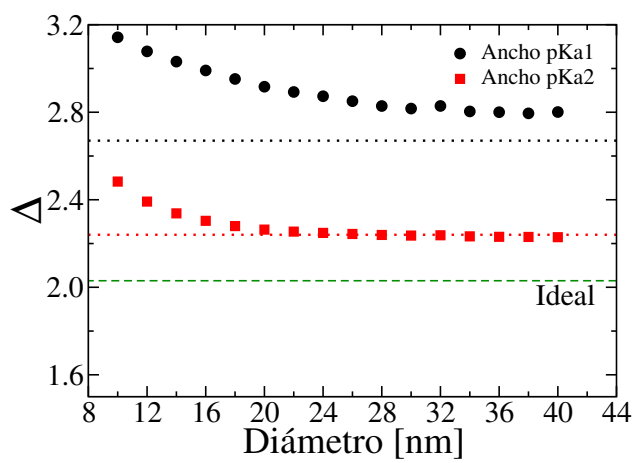

(b)

Figura 5.2.3: (a) $p K a^{a p p}$ como función del diámetro del nanocanal cilíndrico, $D$. El $p K_{a 1}^{a p p}$ esta definido como el valor de $\mathrm{pH}$ donde $\left\langle f_{0}\right\rangle=\left\langle f_{-1}\right\rangle$, y el $p K_{a 2}^{a p p}$ esta definido como el valor de $\mathrm{pH}$ donde $\left\langle f_{-1}\right\rangle=\left\langle f_{-2}\right\rangle$. (b) Ancho de la transición $(\Delta)$ como función de $D$. $\Delta$ está definido como la diferencia entre los valores de $\mathrm{pH}$ donde el grado de disociación alcanza los valores $1 / 11$ y $10 / 11\left(\Delta_{1}\right.$ para $\left\langle f_{-1}\right\rangle$ y $\Delta_{2}$ para $\left.\left\langle f_{-2}\right\rangle\right)$. Las líneas horizontales rayadas muestran los valores de bulk esperados de $p K a$ y $\Delta$. Las horizontales punteadas muestran las predicciones del modelo analítico sencillo ecuaciones $(5.1,5.2,5.3,5.4)$, (C.6, C.7, C.8, C.9). Parámetros: $N_{p} / A(R)=0,2_{\text {cadenas }} / A(R)$, grado de polimerización $N=28, p K_{a 1}^{\text {bulk }}=4,5, p K_{a 2}^{\text {bulk }}=7,7$, concentración de sal $C_{\text {salt }}=0,1 M$.

polielectrolitos aumenta, haciendo que la repulsión electrostática entre los monómeros también aumente. Esta repulsión electrostática puede ser reducida mediante tres estrategias: incorporando contra-iones en el seno del nanocanal (cuyo costo energético es la reducción de la entropía traslacional de estos iones), mediante el estiramiento de los polímeros (cuyo costo energético es la reducción de la entropía conformacional de las cadenas) o produciendo un corrimiento en los equilibrios químicos hacia las especies menos cargadas (cuyo costo energético es un incremento en la energía libre de los equilibrios químicos). El tercero de estos mecanismos (desplazamiento del equilibrio químico) implica que la constante de equilibrio aparente $p K_{a}^{a p p}$ para un poliácido es mayor a la que se encuentra en solución ( $p K_{a}^{\text {sol }}$ en solución)[68]. Como se muestra en trabajos previos[70, 46], todos los mecanismos están activos, aunque el estiramiento de las cadenas es más importante durante la primer deprotonación que durante la segunda, comparar la distribución de polímeros a $p H=2,0$ y $p H=6,0$ en la figura 5.2.1a.

Los equilibrios químicos en confinamiento no sólo están desplazados sino que también muestran transiciones más anchas de las que se encuentran en solución; por eso, para una descripción completa deben considerarse no sólo las constantes de equilibrio $p K_{a}$, sino también el ancho de las transiciones. Definimos el ancho de las transiciones como la diferencia entre los valores de $p H$ donde los grados de disociación alcanzan los valores 1/11 y 10/11 (ver figura C.0.1).

$$
\begin{aligned}
& \Delta_{1}=p H\left(\left\langle f_{-1}\right\rangle=\frac{10}{11}\right)-p H\left(\left\langle f_{-1}\right\rangle=\frac{1}{11}\right) \\
& \Delta_{2}=p H\left(\left\langle f_{-2}\right\rangle=\frac{10}{11}\right)-p H\left(\left\langle f_{-2}\right\rangle=\frac{1}{11}\right)
\end{aligned}
$$

Con esta definición un equilibrio ácido-base en solución tiene un ancho de transición $\Delta=2$. En el caso del monómero MEP en solución, ambas transiciones tendrán un ancho $\Delta=2,03$, levemente distinto al ideal. Esto se debe a la presencia de la segunda transición en el mismo monómero. Las constantes de equilibrio distan entre si, $p K_{a 2}-p K_{a 1}=3,2$ [62] unidades de $\mathrm{pH}$, por lo tanto es esperable un acoplamiento pequeño entre los dos equilibrios ácido-base. 
En las figuras 5.2.3a y 5.2.3b, se reporta el efecto del diámetro de nanocanales cilíndricos sobre los valores de $p K_{a 1}^{a p p}$ y $p K_{a 2}^{a p p}$, y el ancho de las transiciones $\Delta_{1}$ y $\Delta_{2}$. La constantes de equilibrio $p K_{a}^{a p p}$ como los anchos de transición $\Delta$ aumentan al disminuir el diámetro del nanocanal. El $p K_{a}^{a p p}$ difiere del $p K_{a}^{b u l k}$ por que, como se explicó anteriormente, el equilibrio se desplaza hacia las especies menos cargadas para minimizar la repulsión electrostática en el sistema (este proceso se conoce como regulación de carga).

Para entender los efectos del confinamiento sobre las transiciones químicas, se propuso un modelo analítico sencillo, que consiste en una formulación simplificada de la teoría molecular general. Este modelo es útil para entender el desplazamiento de las constantes de equilibrio $p K_{a}$ y el ancho de las transiciones $\Delta$, que se muestran en las figuras 5.2.3a y 5.2.3b. El modelo se describe en profundidad en el apéndice $\mathrm{C}$ allí se considera una capa polimérica homogénea en contacto con una solución homogénea, es decir describe un sistema de dos fases y se asume $f_{-2}=0$ durante la primera transición y $f_{0}=0$ durante la segunda transición. Las expresiones analíticas, ecuaciones 5.1 y 5.2, para el primer y segundo equilibrio requieren los parámetros de entrada: $f_{-1}$ y $f_{-2}$. (las fracciones de disociación de los monómeros de MEP en la capa polimérica), $\phi_{p}$ (fracción de volumen de polímero en la capa, se uso el valor $\phi_{p}=0,053$ para ambas transiciones), y $C_{\text {salt }}$ (concentración de sal en el seno de la solución). Las constantes aparentes $p K_{a}$ pueden ser estimadas como el valor de $\mathrm{pH}$ cuando $f_{-1}=0,5\left(\right.$ para $p K_{a 1}^{a p p}$ ) o $f_{-2}=0,5\left(\right.$ para $\left.p K_{a 2}^{a p p}\right)$.

$$
\begin{aligned}
& p H\left(f_{-1}\right)=p K_{a 1}^{\text {bulk }}+\log _{10}\left(\frac{f_{-1}}{1-f_{-1}}\right)+\log _{10}\left(\frac{1}{0,602 C_{\text {salt }} v_{p}} \frac{f_{-1} \phi_{p}}{\left(1-\phi_{p}\right)^{v_{s} / v_{p}}}\right) \\
& p H\left(f_{-2}\right)=p K_{a 2}^{\text {bulk }}+\log _{10}\left(\frac{f_{-2}}{1-f_{-2}}\right)+\log _{10}\left(\frac{1}{0,602 C_{\text {salt }} v_{p}} \frac{\left(1+f_{-2}\right) \phi_{p}}{\left(1-\phi_{p}\right)^{v_{s} / v_{p}}}\right)
\end{aligned}
$$

Las predicciones del modelo sencillo (líneas punteadas en la figura 5.2.3a) son consistentes con los cálculos de la teoría molecular (símbolos en la figura 5.2.3a) para diámetros grandes $(D>50 \mathrm{~nm})$. Este resultado es importante porque muestra que es posible capturar el desplazamiento de los equilibrios químicos con un modelo sencillo en el rango de diámetros grandes $(D>h)$. Como era esperado, al reducir la concentración de sal aumenta el costo en la energía libre de mantener descargado los monómeros de MEP en la capa polimérica e incrementa el desplazamiento de las constantes aparentes $p K_{a}$ con respecto a los valores de solución. (ver figura C.0.3).

Para radios menores a la longitud máxima de estiramiento de las cadenas poliméricas $(\sim 10 \mathrm{~nm}$ en nuestro caso), el modelo simple falla en la reproducción de las predicciones realizadas por la teoría molecular porque el modelo sencillo no toma en cuenta los efectos de la curvatura de la superficie. En conclusión, para nanocanales angostos donde los equilibrios químicos se encuentran desplazados hacia las especies menos cargadas, los detalles moleculares del polímero son importantes y deben ser tenidos en cuenta.

Las expresiones para los anchos de las transiciones de protonación del modelo sencillo son:

$$
\begin{gathered}
\Delta_{1}=\Delta^{\text {ideal }}+\log _{10}\left(10 \frac{\phi_{p}^{B}}{\left(1-\phi_{p}^{B}\right)^{v_{s} / v_{w}}} \frac{\left(1-\phi_{p}^{A}\right)^{v_{s} / v_{w}}}{\phi_{p}^{A}}\right) \\
\Delta_{1}=\Delta^{\text {ideal }}+\log _{10}\left(\frac{21}{12}\right)=2,24
\end{gathered}
$$

Las ecuaciones 5.3 y 5.4, muestran que el ancho de las transiciones tienen dos contribuciones. 
Una contribución está relacionada con las transiciones en solución, $\Delta^{\text {ideal }}=2$, mientras la otra esta relacionada con la distribución de polímero en la capa polimérica homogénea. $\phi_{p}^{A}$ y $\phi_{p}^{B}$ son las fracciones de volumen de la capa polimérica homogénea cuando $\left\langle f_{-1}\right\rangle=1 / 11 \mathrm{y}\left\langle f_{-1}\right\rangle=10 / 11$, respectivamente. Usamos $\phi_{p}^{B}=0,053$ y estimamos $\phi_{p}^{A}=0,1$ de los resultados de la figura 5.2.2, (los criterios para decidir estos valores se discuten en torno a la figura C.0.2). El ancho de la segunda transición $\Delta_{2}$ es independiente de la fracción de volumen de polímero en la capa polimérica porque asumimos que el segundo paso de deprotonación deja invariante $\phi_{p}\left(=\phi_{p}^{B}\right)$, ver figura 5.2.1a.

La figura 5.2.3b muestra el ancho de las transiciones 0/-1 y -1/-2 para una concentración de sal $C_{\text {salt }}=0,1 M$. La linea rayada horizontal verde indica el ancho de la transición ideal en bulk para un ácido diprótico (2.03 unidades de $\mathrm{pH}$ ), mientras que las líneas punteadas roja y negra muestran el ancho de las transiciones obtenidos por nuestro modelo de capa polimérica homogénea.

Como se discutió, el costo en la energía libre del proceso de protonación de los monómeros de PMEP es mayor dentro del nanocanal que en el seno de la solución debido a las repulsiones electrostáticas entre las cargas negativas de los monómeros. Este costo energético depende de la organización molecular de la capa polimérica, como por ejemplo las conformaciones del polielectrolito o el número de contra-iones dentro del canal. La organización molecular cambia a medida que varía el pH de la solución (ver figuras 5.2.1 y 5.2.2), lo cual resulta en un cambio continuo del costo energético del proceso de protonación y por lo tanto producen transiciones más anchas que las transiciones ideales. En otras palabras la contribución no ideal al ancho de las transiciones, ecuaciones 5.3 y 5.4, tienen su origen en la variación de las interacciones electrostáticas dentro de la capa polimérica durante el proceso de protonación[26, 50, 71, 31]. (Es posible pensar este efecto como una titulación ácido-base donde el $p K_{a}$ efectivo se desplaza con el pH). Es interesante observar también que el ancho de la segunda transición es siempre menor que el ancho de la primera transición. Este resultado se explica con el hecho de que la reorganización del sistema durante la transición 0/-1 es más dramática que durante la transición -1/-2. (ver la concentración de contra-iones en la figura 5.2.1 y los perfiles de la fracción de volumen de polielectrolito en la figura 5.2.2).

\subsection{Equilibrio Ácido-Base y Conductancia en Nanocana- les no Cilíndricos}

En esta sección se comparan los resultados obtenidos para nanocanales cilíndricos con los resultados para canales no cilíndricos. Las geometrías exploradas corresponden a nanocanales que asemejan un cono truncado y nanocanales con perfiles parabólicos con forma de trompeta. Los cálculos para estas geometrías fueron hechos con valores experimentales reportados en la bibliografía: $D_{\min }=10 \mathrm{~nm}$ (diámetro de la punta), $D_{\max }=290 \mathrm{~nm}$ (diámetro de la base), y $L=12 \mu m$ longitud del nanocanal. En todos los casos se investigan nanocanales modificados con brushes polipróticos (PMEP).

Las figuras 5.3.1b1 y 5.3.1b2, muestran la carga promedio por monómero $\left\langle Q_{m o n}\right\rangle$ en función del pH. Los detalles de los cálculos para obtener $\left\langle Q_{m o n}\right\rangle$ pueden leerse en 3.1.1

Los valores de $p K_{a}^{c o n d}$ estimados a partir de las curvas de conductancia y los $p K_{a}^{a p p}$ obtenidos de las curvas de $\left\langle f_{e}\right\rangle$ son diferentes para nanocanales cónicos y tipo trompeta. La tabla 5.1 resume los valores de las constantes de equilibrio obtenidas $p K_{a}^{a p p}, p K_{a}^{\text {cond }}$ y su diferencia $\Delta p K_{a, i}^{a p p}=p K_{a, i}^{c o n d}-p K_{a, i}^{a p p}, i=1,2$ para las distintas formas del nanocanal. Los resultados 

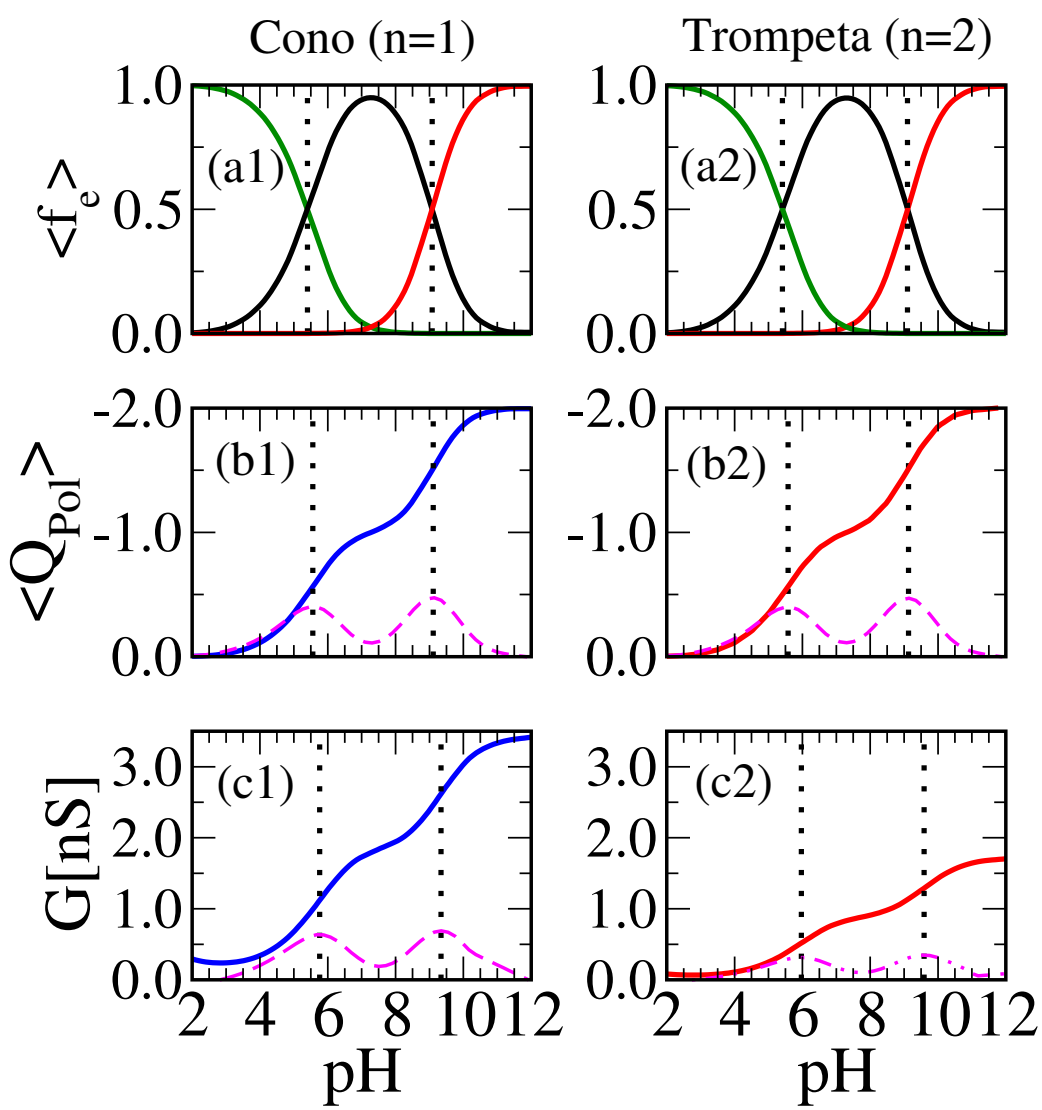

Figura 5.3.1: Se compara el comportamiento de nanocanales con forma de cono $(n=1)$ y forma de trompeta $(n=2)$. El parámetro $n$ aparece en la parametrización de la geometría. Los gráficos $(\mathrm{a} 1, \mathrm{a} 2)$ muestran el grado de disociación promedio $\left\langle f_{e}\right\rangle$ en función del $\mathrm{pH}$ de la solución. Donde $e=0$ corresponde al estado neutro (curva verde), $e=-1$ corresponde al estado con carga negativa (curva negra) y $e=-2$ corresponde al estado con carga -2 (curva roja). Los gráficos (b1,b2) muestran la carga media por monómero como función del pH de la solución; la curva rayada muestra la primer derivada con respecto al $\mathrm{pH}$. (c) Conductancia del nanocanal, $G$, como función del $\mathrm{pH}$; la curva punteada muestra los $p K a^{a p p}$ estimados con las condiciones: $\left\langle f_{0}\right\rangle=\left\langle f_{-1}\right\rangle$ y $\left\langle f_{-1}\right\rangle=\left\langle f_{-2}\right\rangle$ en $(\mathrm{a} 1, \mathrm{a} 2)$ y la primer derivada de $G$ in $(\mathrm{c} 1, \mathrm{c} 2) . D_{\min }=10 \mathrm{~nm}$, $D_{\text {max }}=290 \mathrm{~nm}, L=12 \mu \mathrm{m}, C_{\text {salt }}=0,1 \mathrm{M}$. 
Cuadro 5.1: Resumen de las constantes de equilibrio $p K_{a i}$, para la primera $(i=1)$ y segunda $(i=2)$ transición, determinados a partir de dos métodos distintos: a partir de las curvas de especiación $\left(p K_{a i}^{a p p}\right)$ y a partir de los puntos de inflexión de las curvas de conductancia $G$ vs $p H\left(p K_{a i}^{c o n d}\right)$. Se muestra también la diferencia $\Delta p K_{a i}^{a p p}=p K_{a i}^{c o n d}-p K_{a i}^{a p p}$.

\begin{tabular}{lccc} 
& cilindro & cono & trompeta \\
$p K_{a 1}^{a p p}$ & 5.81 & 5.40 & 5.41 \\
$p K_{a 1}^{c o n d}$ & 6.06 & 5.76 & 5.97 \\
$\Delta p K_{a 1}^{a p p}$ & 0.25 & 0.36 & 0.56 \\
\hline$p K_{a 2}^{a p p}$ & 9.67 & 9.08 & 9.10 \\
$p K_{a 2}^{c o n d}$ & 9.69 & 9.34 & 9.59 \\
$\Delta p K_{a 2}^{a p p}$ & 0.02 & 0.26 & 0.49
\end{tabular}

muestran que la geometría del nanocanal cambia levemente la concordancia entre los $p K_{a}$ aparentes obtenidos con mediciones macroscópicas vía la conductancia $\left(p K_{a, i}^{c o n d}\right)$ y la que se obtiene directamente de los estados de protonación del sistema, $p K_{a, i}^{a p p}$. Esta concordancia es importante para la caracterización experimental de nanocanales con distintas formas. La discrepancia entre $p K_{a}^{a p p}$ y $p K_{a}^{c o n d}$ en nanocanales no cilíndricos se debe al hecho de que la conductancia es más sensible al estado de protonación del polielectrolito en la punta del nanocanal que en la base. Es por esto que la conductancia no refleja el estado de carga global de nanocanales no cilíndricos. La tabla 5.1 también muestra que las constantes aparentes $p K_{a}^{a p p}$ para los nanocanales tipo trompeta son más grandes (el corrimiento respecto de los valores en el seno de la solución es mayor) que en el caso de los nanocanales cónicos. Esto puede explicarse con el hecho de que las geometrías tipo trompeta son más angostas que las geometrías cónicas para todo valor de $z$ por lo que el peso relativo de los diámetros pequeños es mayor en las geometrías tipo trompeta. Como mostramos en la figura 5.2.3a los valores de las constantes $p K_{a}^{a p p}$ se incrementan al disminuir el radio del nanocanal. 


\section{Capítulo 6}

\section{Conclusiones}

En este trabajo desarrollamos un modelo para nanocanales modificados que incorpora el detalle químico y físico de las moléculas que modifican el nanocanal. Esta metodología denominada teoría molecular, encuentra su lugar en el espectro de metodologías teórico/computacionales entre las simulaciones computacionales (atomísticas y de grano grueso) y los modelos continuos (como las ecuaciones de Poisson-Nernst-Planck, PNP). La teoría molecular es especialmente útil porque permite hallar la organización molecular en condiciones de equilibrio termodinámico. Uno de los aportes principales de esta tesis consiste es el desarrollo de una metodología teórica basada en los resultados de la teoría molecular para nanocanales cilíndricos largos. El método desarrollado es útil en geometrías que tienen simetría azimutal (homogéneos en la dirección angular $\hat{\theta}$ ) pero que pueden incorporar asimetrías en la dirección $\hat{z}$. Se investigaron primero sistemas simples para contrastar los resultados de esta metodología con otras técnicas: resolución de las ecuaciones PNP, ver comparación en la figura 3.1.3 y resultados experimentales ver figura 3.1.4. Con esta nueva metodología se investigó el efecto del confinamiento sobre brushes de polielectrolitos (Cap. 5) y la funcionalización de nanocanales asimétricos por adsorción de polielectrolitos (Cap. 4).

En el caso de brushes poliméricos, los equilibrios ácido-base presentes en las unidades monoméricas del PMEP (poli(2-(metacriloiloxi) etil-fosfato) en confinamiento no sólo exhiben un desplazamiento respecto del comportamiento en el seno de la solución, sino que también muestran transiciones más anchas de las que se encuentran en solución. Por eso, mostramos que para una descripción completa deben considerarse no sólo las constantes de equilibrio $p K_{a}$, sino también el ancho de las transiciones.

La organización molecular dentro de nanocanales modificados con brushes poliméricos es sensible a la curvatura del nanocanal. Para diámetros grandes desarrollamos un modelo sencillo de capa polimérica homogénea cuyos resultados son compatibles con los obtenidos con la teoría más general. Este resultado es importante porque muestra que es posible capturar el desplazamiento de los equilibrios químicos con un modelo sencillo en el rango de diámetros grandes $(D>h)$. Observamos que a medida que disminuye el diámetro del nanocanal, el nivel de acuerdo entre la teoría molecular y el modelo de capa homogénea también disminuye. Para nanocanales angostos $D<40 \mathrm{~nm}$, el modelo sencillo falla en la reproducción de las predicciones realizadas por la teoría molecular porque no considera los efectos de la curvatura de la superficie. En conclusión, para nanocanales angostos donde los equilibrios químicos se encuentran desplazados hacia las especies menos cargadas, los detalles moleculares del polímero son importantes y deben ser tenidos en cuenta. Los resultados presentados en el capítulo 5 son de interés para la interpretación de las curvas de conductancia en función del pH, así como para el diseño 
de nanocanales modificados con propiedades geométricas, físicas y químicas específicas.

Cuando la modificación química del nanocanal implica la adsorción de polielectrolitos (Cap. 4), debe modificarse la energía libre del sistema para incorporar los grados de libertad de traslación de las cadenas poliméricas. Si los experimentos de adsorción del polilectrolito dentro del nanocanal ocurren bajo la condición de equilibrio termodinámico, entonces el potencial químico de las cadenas debe ser constante, $\mu_{P}=c t e$. Hemos hallado dos resultados interesantes respecto del comportamiento del sistema bajo esta condición. En primer lugar, la adsorción es homogénea sólo en el caso de nanocanales cilíndricos. En segundo lugar, cuando la geometría del nanocanal es asimétrica la adsorción es en general inhomogénea y depende de la concentración de sal y de polielectrolitos en solución. Como se investigó en el capítulo 4, dependiendo de la concentración de sal y la concentración del polielectrolito, a medida que el diámetro disminuye (aumenta la curvatura y el confinamiento) la densidad de cadenas por unidad de superficie puede aumentar, disminuir o permanecer constante. Este abanico de posibilidades es el resultado del balance entre la energía electrostática y la entropía traslacional total de los iones y el polielectrolito. La condición de electroneutralidad entre la carga de la pared, la carga del polilectrolito y la carga iónica proveniente de la sal, se desplaza hacia la adsorción de polielectrolitos cuando la concentración de sal es baja y hacia la adsorción de iones provenientes de la sal para concentraciones altas de sal. Al aumentar el confinamiento (diámetros chicos) el sistema siempre prefiere disminuir el exceso de carga efectiva sobre la pared.

Aunque otros marcos teóricos (por ejemplo, las ecuaciones de PNP) pueden considerar inhomogeneidades en la carga efectiva sobre la pared del nanocanal, ninguno provee pista alguna acerca de la forma de la distribución de cargas. Consideramos que los resultados de este trabajo es de especial relevancia porque hasta donde sabemos es la primera vez que se reportan resultados teóricos que cuantifican las inhomogeneidades en la distribución de carga dentro del nanocanal.

Los resultados preliminares basados en la teoría molecular, indican que la distribución de cargas dentro del canal podría controlarse con la concentración de sal en solución durante la modificación de nanocanales por adsorción de polielectrolitos. Este fenómeno de adsorción inhomogénea resulta relevante, dada la relación que existe entre la rectificación de nanocanales asimétricos y la carga superficial efectiva de las paredes del nanocanal.

En el transcurso de este trabajo han quedado pendientes algunos modelos y sistemas que consideramos relevantes para el futuro de la investigación teórico/computacional en el área. Por un lado, el estudio de la conductancia de nanocanales con geometría bullet-like, modificados con polielectrolitos adsorbidos en su superficie. Estos sistemas son estudiados bajo la hipótesis de distribución de carga homogénea, en términos de sus propiedades dinámicas, por ejemplo, el grado de rectificación. Sin embargo, estas propiedades emergen del ordenamiento molecular y su respuesta frente al potencial externo. Consideramos que la planificación de experimentos de conductancia combinados con las herramientas teóricas desarrolladas en este trabajo, permitirían conocer las propiedades estructurales de estos sistemas. Por otro lado, en el capítulo 2, mostramos que es posible incorporar distintas arquitecturas y entidades químicas a las cadenas poliméricas, ver figura 2.2.1. Esta versatilidad de la teoría molecular permitiría el estudio de nanocanales modificados con polímeros zwitteriónicos e incluso polímeros ramificados. Estos sistemas también pueden ser modelados con el marco teórico desarrollado.

Una de las limitaciones de este marco teórico es la pérdida de los efectos hidrodinámicos, como el flujo electro-osmótico. En condiciones de cuasi-equilibrio, como las consideradas en el estudio de nanocanales largos, las corrientes iónicas son pequeñas y las contribuciones a la electro-ósmosis pueden ser despreciadas. Otra de las limitaciones de la teoría es la elección 
de los coeficientes de difusión, que son valores de entrada en el cálculo de conductancia en condición de cuasi-equilibrio . Nosotros aproximamos el coeficiente de difusión dentro del canal, por aquellos valores dados en el seno de la solución, fundamentados en el alto contenido de agua (alrededor del $80 \%$ ) dentro del nanocanal. Esta aproximación parece funcionar correctamente considerando el nivel de acuerdo entre los resultados teóricos y experimentales, figuras 3.1.3, 3.1.4 y 5.1.1. Sin embargo, es deseable desarrollar un método consistente para la estimación de los coeficientes de difusión en los nanocanales modificados. Una opción susceptible de ser estudiada en el futuro es el uso de simulaciones de dinámica molecular para determinar el coeficiente de difusión dentro de la capa de polielectrolitos.

Finalmente, consideramos que el desarrollo de nuevas plataformas de control del transporte iónico, a través de nanocanales modificados químicamente es un desafío que requiere entender las propiedades en confinamiento. En este trabajo hemos desarrollado modelos con los cuales exploramos las propiedades de polielectrolitos confinados. Gracias a la versatilidad de estos modelos es posible investigar una multiplicidad de sistemas realizables experimentalmente. Una herramienta teórico/computacional como la desarrollada en este trabajo permite explicar la respuesta macroscópica en función de las características moleculares, y guiar a los experimentalistas en la interpretación y la predicción de nuevos resultados. 


\section{Bibliografía}

[1] Mubarak Ali, Salvador Mafe, Patricio Ramirez, Reinhard Neumann, and Wolfgang Ensinger. Logic gates using nanofluidic diodes based on conical nanopores functionalized with polyprotic acid chains. 25(20):11993-11997. 8

[2] Mubarak Ali, Saima Nasir, Quoc Hung Nguyen, Jugal Kishore Sahoo, Muhammad Nawaz Tahir, Wolfgang Tremel, and Wolfgang Ensinger. Metal ion affinity-based biomolecular recognition and conjugation inside synthetic polymer nanopores modified with ironterpyridine complexes. 133(43):17307-17314. 8

[3] Mubarak Ali, Saima Nasir, Patricio Ramirez, Javier Cervera, Salvador Mafe, and Wolfgang Ensinger. Carbohydrate-Mediated Biomolecular Recognition and Gating of Synthetic Ion Channels. The Journal of Physical Chemistry C, 117(35):18234-18242, sep 2013. 8

[4] Mubarak Ali, Patricio Ramirez, Hung Quoc Nguyen, Saima Nasir, Javier Cervera, Salvador Mafe, and Wolfgang Ensinger. Single cigar-shaped nanopores functionalized with amphoteric amino acid chains: Experimental and theoretical characterization. 6(4):3631-3640. 8

[5] Mubarak Ali, Patricio Ramirez, Muhammad Nawaz Tahir, Salvador Mafe, Zuzanna Siwy, Reinhard Neumann, Wolfgang Tremel, and Wolfgang Ensinger. Biomolecular conjugation inside synthetic polymer nanopores viaglycoprotein-lectin interactions. Nanoscale, 3:18941903, 2011. 8

[6] Mubarak Ali, Basit Yameen, Reinhard Neumann, Wolfgang Ensinger, Wolfgang Knoll, and Omar Azzaroni. Biosensing and supramolecular bioconjugation in single conical polymer nanochannels. Facile incorporation of biorecognition elements into nanoconfined geometries. Journal of the American Chemical Society, 130(48):16351-7, dec 2008. 12

[7] P Apel, A Schulz, and R Spohr. Track size and track structure in polymer irradiated by heavy ions. Nuclear Instruments and ..., 146:468-474, 1998. 13, 36

[8] P. Yu Apel, I. V. Blonskaya, N. V. Levkovich, and O. L. Orelovich. Asymmetric track membranes: Relationship between nanopore geometry and ionic conductivity. Petroleum Chemistry, 51(7):555-567, December 2011. 36, 39

[9] Pavel Y. Apel, Valery V. Bashevoy, Irina V. Blonskaya, Nikolay E. Lizunov, Oleg L. Orelovitch, and Christina Trautmann. Shedding light on the mechanism of asymmetric track etching: an interplay between latent track structure, etchant diffusion and osmotic flow. Phys. Chem. Chem. Phys., 18:25421-25433, 2016. 36

[10] A Ben-Shaul and I Szleifer. Chain organization and thermodynamics in micelles and bilayers. 2. Model calculations. J. Chem. Phys., 83:3612, 1985. 14 
[11] A. Ben-Shaul, I. Szleifer, and W. M. Gelbart. Chain organization and thermodynamics in micelles and bilayers. i. theory. 83(7):3597-3611. 14

[12] John C. Hulteen and Charles R. Martin. A general template-based method for the preparation of nanomaterials. J. Mater. Chem., 7:1075-1087, 1997. 8

[13] M. A. Carignano and I. Szleifer. Statistical thermodynamic theory of grafted polymeric layers. 98(6):5006-5018. 14

[14] N. Chtanko, M. E. Toimil Molares, T. Cornelius, D. Dobrev, and R. Neumann. Etched single-ion-track templates for single nanowire synthesis. 108(28):9950-9954. 8

[15] Honggu Chun and Taek Dong Chung. Iontronics. 8(1):441-462. 12

[16] B. Corry, S. Kuyucak, and S.H. Chung. Invalidity of continuum theories of electrolytes in nanopores. Chemical Physics Letters, 320(1-2):35 - 41, 2000. 11

[17] Ben Corry, Serdar Kuyucak, and Shin-Ho Chung. Tests of continuum theories as models of ion channels. ii. poisson-nernst-planck theory versus brownian dynamics. Biophysical Journal, 78(5):2364 - 2381, 2000. 11, 34

[18] Hirofumi Daiguji, Peidong Yang, Andrew J. Szeri, and Arun Majumdar. Electrochemomechanical Energy Conversion in Nanofluidic Channels. Nano Letters, 4(12):2315-2321, December 2004. 61

[19] L. Dauginet, A.-S. Duwez, R. Legras, and S. Demoustier-Champagne. Surface modification of polycarbonate and poly(ethylene terephthalate) films and membranes by polyelectrolyte deposition. 17(13):3952-3957. 8

[20] Pierre-Gilles de Gennes. Nobel lecture: Soft matter. The Nobel Foundation, December 9 1991. 5

[21] Gero Decher. Fuzzy nanoassemblies: Toward layered polymeric multicomposites. Science, 277(5330):1232-1237, 1997. 9

[22] Joshua B. Edel and Tim Albrecht. Engineered Nanopores for Bioanalytical Applications (Micro and Nano Technologies). William Andrew, 2013. 9

[23] Jan C. T. Eijkel and Albert van den Berg. Nanofluidics and the chemical potential applied to solvent and solute transport. Chem. Soc. Rev., 39:957-973, 2010. 42

[24] P. G. de Gennes. Scaling Concepts in Polymer Physics. Cornell University Press, Ithaca. 6

[25] Facundo M. Gilles, Mario Tagliazucchi, Omar Azzaroni, and Igal Szleifer. Ionic conductance of polyelectrolyte-modified nanochannels: Nanoconfinement effects on the coupled protonation equilibria of polyprotic brushes. The Journal of Physical Chemistry C, 120(9):47894798, 2016. 52,85

[26] Dirk Gillespie and Robert Eisenberg. Modified Donnan potentials for ion transport through biological ion channels. Physical Review E, 63(6):061902, may 2001. 65

[27] DE Goldman. Potential, impedance, and rectification in membranes. The Journal of General Physiology, 1943. 34 
[28] Peng Gong and I. Szleifer. Competitive adsorption of model charged proteins: the effect of total charge and charge distribution. Journal of Colloid and Interface Science, 278(1):81 $-90,2004.28$

[29] Gaurav Goyal, Armin Darvish, and Min Jun Kim. Use of solid-state nanopores for sensing co-translocational deformation of nano-liposomes. Analyst, 140:4865-4873, 2015. 12

[30] Cuiping Han, Xu Hou, Huacheng Zhang, Wei Guo, Haibing Li, and Lei Jiang. Enantioselective recognition in biomimetic single artificial nanochannels. 133(20):7644-7647. 8

[31] Mitsuru Higa, Akihiko Tanioka, and Akira Kira. A novel measurement method of Donnan potential at an interface between a charged membrane and mixed salt solution. Journal of Membrane Science, 140(2):213-220, March 1998. 65

[32] Terrell L Hill. An Introduction to Statistical Thermodynamics (Dover Books on Physics). Dover Publications, jan 1987. 16, 80

[33] Alan C. Hindmarsh, Peter N. Brown, Keith E. Grant, Steven L. Lee, Radu Serban, Dan E. Shumaker, and Carol S. Woodward. Sundials: Suite of nonlinear and differential/algebraic equation solvers. ACM Trans. Math. Softw., 31(3):363-396, September 2005. 14

[34] Xu Hou, Wei Guo, Fan Xia, Fu-Qiang Nie, Hua Dong, Ye Tian, Liping Wen, Lin Wang, Liuxuan Cao, Yang Yang, Jianming Xue, Yanlin Song, Yugang Wang, Dongsheng Liu, and Lei Jiang. A biomimetic potassium responsive nanochannel: G-quadruplex DNA conformational switching in a synthetic nanopore. 131(22):7800-7805. 8

[35] Xu Hou, Yujie Liu, Hua Dong, Fu Yang, Lin Li, and Lei Jiang. A pH-gating ionic transport nanodevice: Asymmetric chemical modification of single nanochannels. 22(22):2440-2443. 8

[36] Xu Hou, Fu Yang, Lin Li, Yanlin Song, Lei Jiang, and Daoben Zhu. A biomimetic asymmetric responsive single nanochannel. 132(33):11736-11742. 8

[37] Xu Hou, Huacheng Zhang, and Lei Jiang. Building bio-inspired artificial functional nanochannels: From symmetric to asymmetric modification. 51(22):5296-5307. 8

[38] Sue Carter Janelle Leger, Magnus Berggren, editor. Iontronics: Ionic Carriers in Organic Electronic Materials and Devices. CRC Press, 2016. 12

[39] J.F. Joanny. Polyelectrolyte adsorption and charge inversion. The European Physical Journal B, 9(1):117-122, May 1999. 6

[40] Rohit Karnik, Kenneth Castelino, Rong Fan, Peidong Yang, and Arun Majumdar. Effects of biological reactions and modifications on conductance of nanofluidic channels. 5(9):16381642. 9

[41] Cornelius Lanczos. The variational principles of mechanics. Dover Publications, New York, 1986. 14

[42] Ira N Levine. FisicoquÃmica Vol.1. McGraw-Hill Interamericana, New York, 1997. 15

[43] David R. Lide. CRC handbook of chemistry and physics a Ready-Reference Book of Chemical and Physical Data. CRC press inc., Boca Raton; Ann Arbor; London [etc.], 1991. 21 
[44] Gabriel Longo and I Szleifer. Ligand-receptor interactions in tethered polymer layers. Langmuir : the ACS journal of surfaces and colloids, 21(24):11342-51, nov 2005. 14

[45] M. Muthukumar. Polymer Translocation. CRC Press, April 2016. 10

[46] R Nap, Peng Gong, and Igal Szleifer. Weak polyelectrolytes tethered to surfaces: effect of geometry, acid-base equilibrium and electrical permittivity. Journal of Polymer Science Part B: ..., 44:2638-2662, 2006. 14, 28, 63

[47] R Nap and I Szleifer. Control of Carbon Nanotube-Surface Interactions: The Role of Grafted Polymers. Langmuir, 21:12072-12075, 2005. 14

[48] Rikkert J Nap, M Tagliazucchi, and I Szleifer. Born energy, acid-base equilibrium, structure and interactions of end-grafted weak polyelectrolyte layers. The Journal of chemical physics, 140(2):024910, jan 2014. 14

[49] Roland R. Netz and David Andelman. Neutral and charged polymers at interfaces. Physics Reports, 380(1-2):1-95, jun 2003. 9

[50] H Ohshima and S Ohki. Donnan potential and surface potential of a charged membrane. Biophysical journal, 47(5):673-8, May 1985. 65

[51] Gonzalo Perez-Mitta, Alberto G. Albesa, Christina Trautmann, Maria Eugenia ToimilMolares, and Omar Azzaroni. Bioinspired integrated nanosystems based on solid-state nanopores: iontronic"transduction of biological, chemical and physical stimuli. Chem. Sci., 8:890-913, 2017. 12

[52] Gonzalo Pérez-Mitta, Jimena S. Tuninetti, Wolfgang Knoll, Christina Trautmann, María Eugenia Toimil-Molares, and Omar Azzaroni. Polydopamine Meets Solid-State Nanopores: A Bioinspired Integrative Surface Chemistry Approach To Tailor the Functional Properties of Nanofluidic Diodes. Journal of the American Chemical Society, page 150430122659003, 2015. 13

[53] Susan Perkin, Ronit Goldberg, Liraz Chai, Nir Kampf, and Jacob Klein. Dynamic properties of confined hydration layers. Faraday Discuss., 141:399-413, 2009. 6

[54] Carine Perrot, Laurent Gonon, Catherine Marestin, and GÃ@rard Gebel. Hydrolytic degradation of sulfonated polyimide membranes for fuel cells. 379(1):207-214. 8

[55] P. Ramírez, P. Yu Apel, J. Cervera, and S. Mafé. Pore structure and function of synthetic nanopores with fixed charges: tip shape and rectification properties. Nanotechnology, 19(31):315707, August 2008. 36

[56] Reto Schoch, Jongyoon Han, and Philippe Renaud. Transport phenomena in nanofluidics. Reviews of Modern Physics, 80(3):839-883, jul 2008. 90

[57] Z. Siwy, I. D. Kosińska, A. Fuliński, and C. R. Martin. Asymmetric diffusion through synthetic nanopores. 94(4). 8

[58] ZS Siwy, D. Dobrev, R. Neumann, C. Trautmann, and K. Voss. Electro-responsive asymmetric nanopores in polyimide with stable ion-current signal. Applied Physics A, 76(5):781785, mar 2003. 8 
[59] Zuzanna S. Siwy, Pavel Apel, Dagmar Baur, and DD Dobrev. Preparation of synthetic nanopores with transport properties analogous to biological channels. Surface Science, 532-535:1061-1066, jun 2003. 8

[60] Zuzanna S Siwy and Stefan Howorka. Engineered voltage-responsive nanopores. Chemical Society reviews, 39(3):1115-32, mar 2010. 12

[61] Zuzanna S. Siwy, Lacramioara Trofin, Punit Kohli, Lane a Baker, Christina Trautmann, and Charles R Martin. Protein biosensors based on biofunctionalized conical gold nanotubes. Journal of the American Chemical Society, 127(14):5000-1, apr 2005. 9

[62] Douglas A. Skoog, Donald M. West West, F. James Holler, and Stanley R. Crouch Crouch. Fundamentals Of Analytical Chemistry. Saunders College Pub, 7 edition, 1995. 63

[63] R. Spohr. Ion Tracks and Microtechnology: Principles and Applications. Vieweg+Teubner Verlag, 2013. 8

[64] Zhongyue Sun, Cuiping Han, Miaomiao Song, Long Wen, Demei Tian, Haibing Li, and Lei Jiang. Fabrication of cysteine-responsive biomimetic single nanochannels by a thiol-yne reaction strategy and their application for sensing in urine samples. 26(3):455-460. 8

[65] Mario Tagliazucchi Igal Szleifer, editor. Elsevier, 2016. 8, 12

[66] Mario Tagliazucchi, Omar Azzaroni, and Igal Szleifer. Responsive polymers end-tethered in solid-state nanochannels: when nanoconfinement really matters. Journal of the American Chemical Society, 132(35):12404-11, September 2010. 35, 58, 61

[67] Mario Tagliazucchi, Yitzhak Rabin, and Igal Szleifer. Transport rectification in nanopores with outer membranes modified with surface charges and polyelectrolytes. ACS Nano, 7(10):9085-9097, 2013. 38

[68] Mario Tagliazucchi and Igal Szleifer. Stimuli-responsive polymers grafted to nanopores and other nano-curved surfaces: structure, chemical equilibrium and transport. Soft Matter, 8(28):7292, 2012. 63

[69] Ivan Vlassiouk, Sergei Smirnov, and Zuzanna S. Siwy. Ionic selectivity of single nanochannels. Nano letters, 8(7):1978-85, jul 2008. 34

[70] Dawei Wang, Rikkert J Nap, István Lagzi, Bartlomiej Kowalczyk, Shuangbing Han, Bartosz a Grzybowski, and Igal Szleifer. How and why nanoparticle's curvature regulates the apparent pKa of the coating ligands. Journal of the American Chemical Society, 133(7):2192-7, February 2011. 63

[71] Tzu-Yu Wang, Yu-Jane Sheng, and Heng-Kwong Tsao. Donnan potential of dilute colloidal dispersions: Monte Carlo simulations. Journal of colloid and interface science, 340(2):192201, December 2009. 65

[72] Yajun Wang, Alexandra S. Angelatos, Dave E. Dunstan, and Frank Caruso. Infiltration of macromolecules into nanoporous silica particles. 40(21):7594-7600. 9

[73] Yajun Wang, Vipul Bansal, Alexander N. Zelikin, and Frank Caruso. Templated synthesis of single-component polymer capsules and their application in drug delivery. 8(6):17411745. 9 
[74] Ruoshan Wei, Volker Gatterdam, Ralph Wieneke, Robert Tamp ̃̃®, and Ulrich Rant. Stochastic sensing of proteins with receptor-modified solid-state nanopores. 7(4):257-263. 12

[75] Liping Wen, Xu Hou, Ye Tian, Jin Zhai, and Lei Jiang. Bio-inspired Photoelectric Conversion Based on Smart-Gating Nanochannels. Advanced Functional Materials, 20(16):26362642, aug 2010. 12

[76] Ganhua Xie, Liping Wen, and Lei Jiang. Biomimetic smart nanochannels for power harvesting. Nano Research, 9(1):59-71, January 2016. 35

[77] Basit Yameen, Mubarak Ali, Reinhard Neumann, Wolfgang Ensinger, Wolfgang Knoll, and Omar Azzaroni. Ionic transport through single solid-state nanopores controlled with thermally nanoactuated macromolecular gates. Small, 5(11):1287-1291, jun 2009. 13

[78] Basit Yameen, Mubarak Ali, Reinhard Neumann, Wolfgang Ensinger, Wolfgang Knoll, and Omar Azzaroni. Single Conical Nanopores Displaying pH-Tunable Rectifying Characteristics . Manipulating Molecular Transport with Zwitterionic Polymer Brushes (SI). Journal of the American Chemical Society, 131(6):2070-1, feb 2009. 13

[79] Basit Yameen, Mubarak Ali, Reinhard Neumann, Wolfgang Ensinger, Wolfgang Knoll, and Omar Azzaroni. Synthetic proton-gated ion channels via single solid-state nanochannels modified with responsive polymer brushes. Nano letters, 9(7):2788-93, July 2009. 8, 13

[80] Basit Yameen, Mubarak Ali, Reinhard Neumann, Wolfgang Ensinger, Wolfgang Knoll, and Omar Azzaroni. Proton-regulated rectified ionic transport through solid-state conical nanopores modified with phosphate-bearing polymer brushes. Chemical communications (Cambridge, England), 46(11):1908-10, March 2010. 8, 13, 57

[81] Huacheng Zhang, Xu Hou, Lu Zeng, Fu Yang, Lin Li, Dadong Yan, Ye Tian, and Lei Jiang. Bioinspired Arti fi cial Single Ion Pump. 2013. 8

[82] Minghui Zhang, Zheyi Meng, Jin Zhai, and Lei Jiang. Photo-induced current amplification in L-histidine modified nanochannels based on a highly charged photoacid in solution. Chemical communications (Cambridge, England), 49(23):2284-6, mar 2013. 12

[83] Zhen Zhang, Xiang-Yu Kong, Ganhua Xie, Pei Li, Kai Xiao, Liping Wen, and Lei Jiang. "uphill" cation transport: A bioinspired photo-driven ion pump. Science Advances, 2(10), 2016. 12 


\section{Apéndice A}

\section{Tablas de Símbolos y Valores}

Cuadro A.1: Lista de variables para descripción del polímero

\begin{tabular}{|c|c|}
\hline$N_{p}$ & $\begin{array}{l}\text { Número de cadenas poliméricas pegadas a la } \\
\text { superficie del nanocanal. Se utiliza en el caso de } \\
\text { cepillos poliméricos. }\end{array}$ \\
\hline$n_{\text {mon }}$ & $\begin{array}{l}\text { Número de segmentos (monómeros) en cada cadena } \\
\text { polimérica }\end{array}$ \\
\hline$v_{p}$ & Volumen de un monómero en $\mathrm{nm}^{3}$ \\
\hline$l$ & $\begin{array}{l}\text { Longitud de unión entre dos monómeros se utilizó } \\
\text { el valor } l=0,5 \mathrm{~nm}\end{array}$ \\
\hline$\alpha$ & $\begin{array}{l}\text { Índice para una configuración de cadena: } \\
\text { distribución espacial de los monómeros } \\
\text { pertenecientes a la misma cadena polimérica. }\end{array}$ \\
\hline$P(\alpha), P(r, \alpha)$ & $\begin{array}{l}\text { Probabilidad de la configuración de cadena anclada } \\
\text { a la superficie del nanocanal y cadenas libres }\end{array}$ \\
\hline $\begin{array}{c}\phi_{p}(r)=\frac{N_{P} v_{p}}{A(R)} \sum_{\alpha} n(r, \alpha) P(\alpha) \\
\phi_{p}(r)=\rho_{P C}(r) \sum_{\alpha} \int n\left(r ; r^{\prime}, \alpha\right) P\left(r^{\prime}, \alpha\right)\left(\frac{r}{R}\right) d r\end{array}$ & $\begin{array}{l}\text { Fracción de volumen ocupada por monómeros del } \\
\text { polímero. La densidad de monómeros es } \\
\rho_{p}(r)=\phi_{P}(r) / v_{P}\end{array}$ \\
\hline$\rho_{P C}(r)$ & $\begin{array}{l}\text { Densidad numérica de cadenas. Unidades: } \\
\frac{\text { Número de cadenas }}{n m^{3}}\end{array}$ \\
\hline
\end{tabular}


Cuadro A.2: Símbolos de propiedades Fisicoquímicas relevantes

\begin{tabular}{|c|c|}
\hline & Descripción \\
\hline \hline$\beta \mu_{i}$ & $\begin{array}{r}\text { Potencial químico de la especie indicada en el subíndice en } \\
\text { unidades de energía térmica } \beta=\frac{1}{k_{B} T}\end{array}$ \\
\hline$\beta \mu_{i}^{0}$ & $\begin{array}{c}\text { Potencial químico estándar de la especie indicada en el subíndice } \\
\text { en unidades de energía térmica } \beta=\frac{1}{k_{B} T}\end{array}$ \\
\hline$\phi_{i}(r)$ & Fracción de volumen de la especie indicada con subíndice \\
\hline$\rho_{i}(r)$ & $\begin{array}{c}\text { Densidad de unidades de la especie indicada en el subíndice. } \\
{\left[\frac{1}{n m^{3}}\right]}\end{array}$ \\
\hline Geometría & $\begin{array}{c}\text { Área lateral interna del nanocanal cilíndrico en nanómetros } \\
\text { cuadrados }\left[n m^{2}\right] . \text { La longitud } L \text { se reemplaza por la longitud } \\
\text { diferencial } \delta_{z} \text { y luego se integra para calcular el área interna de } \\
\text { nanocanales no cilíndricos. (Ver las expresiones 2.19, y 4.2, para } \\
\left.\left\langle\rho_{P}(r)\right\rangle\right)\end{array}$ \\
\hline$A(R)=2 \pi R L$ & $\begin{array}{c}\text { Distancia radial al centro del nanocanal se utilizan coordenadas } \\
\text { polares como se indica en la figura 2.0.1. }\end{array}$ \\
\hline$R, R(z)$ & Radio del nanoporo. \\
\hline
\end{tabular}

Cuadro A.3: Tabla de volúmenes utilizados en el programa principal. A partir de estos volúmenes se calcularon los radios listados en la última columna.

\begin{tabular}{ccll}
\hline Descripción & Variable & Valor $\left[\mathrm{nm}^{3}\right]$ & Radio $[\mathrm{nm}]$ \\
\hline \hline Volumen Agua & $v_{w}$ & 0.03 & 0.193 \\
\hline \hline Volumen H+ & $v_{H^{+}}$ & 0.03 & 0.193 \\
\hline \hline Volumen OH- & $v_{\mathrm{OH}^{-}}$ & 0.03 & 0.193 \\
\hline \hline \multicolumn{4}{l}{} \\
\hline \hline Volumen K+ & $v_{K^{+}}$ & 0.0824 & 0.270 \\
\hline \hline Volumen Cl- & $v_{\mathrm{Cl}^{-}}$ & 0.0824 & 0.270 \\
\hline \hline \multicolumn{4}{c}{} \\
\hline \hline Volumen PAH & $v_{p o l}$ & 0.095 & 0.283 \\
\hline \hline Volumen PMEP & $v_{p o l}$ & 0.095 & 0.283 \\
\hline
\end{tabular}

Cuadro A.4: Valores de las mobilidades de las distintas especies iónicas presentes en el sistema. Estos valores se utilizaron en el programa para calcular la conductancia del nanocanal.

\begin{tabular}{|c|c|}
\hline Mobilidad & Valores \\
\hline \hline$\mu_{K}$ & $7,352 \frac{m^{2} S}{m o l}$ \\
\hline$\mu_{C l}$ & $7,634 \frac{m^{2} S}{m^{2}}$ \\
\hline$\mu_{H}$ & $34,982 \frac{m^{2} S}{m o l}$ \\
\hline$\mu_{O H}$ & $19,8 \frac{m^{2} S}{m o l}$ \\
\hline
\end{tabular}


Cuadro A.5: Tabla de magnitudes utilizadas en el programa principal.

\begin{tabular}{|c|c|c|}
\hline Descripción & Variable & \\
\hline \hline Energía térmica & $k_{B} T$ & $25,7 \mathrm{meV}$ \\
\hline Longitud de Bjerrum & $\lambda_{B}$ & $0,716 \mathrm{~nm}$ \\
\hline Carga del electrón & $e^{-}$ & $-1,6021766208 \times 10^{-19} \mathrm{C}$ \\
\hline Constante de Boltzmann & $k_{B}$ & $1,38064852 \times 10^{-23} \mathrm{~J} / \mathrm{K}$ \\
& & $\left(8,6173303 \times 10^{-5} \mathrm{eV} / \mathrm{K}\right)$ \\
\hline
\end{tabular}




\section{Apéndice B}

\section{Soluciones de Bulk}

Dadas las reacciones químicas presentes en los monómeros (2-(metacriloiloxi) etil-fosfato (MEP) se definen las constantes de equilibrio químico $K_{1}$ y $K_{2}$ asociadas,

$$
\begin{gathered}
P H_{2} \rightleftharpoons \mathrm{PH}^{-}+H^{+}, \quad K_{1}=\frac{\left[P H^{-}\right]\left[H^{+}\right]}{\left[P H_{2}\right]} \\
P H^{-} \rightleftharpoons P^{2-}+H^{+}, \quad K_{2}=\frac{\left[P^{2-}\right]\left[H^{+}\right]}{\left[P H^{-}\right]}
\end{gathered}
$$

estas constantes pueden ser medidas y sus valores se encuentran en la literatura. Por otra parte, la termodinámica permite relacionar el cambio en la energía libre de Gibbs con las constantes de equilibrio químico(ver por ejemplo Cap.10[32]). Por definición,

$$
\begin{gathered}
\Delta G_{1}^{0}=\mu_{-1}^{0}+\mu_{H^{+}}^{0}-\mu_{0}^{0}=-\ln \left(K_{1}^{\text {theo }}\right) \\
\Delta G_{2}^{0}=\mu_{-2}^{0}+\mu_{H^{+}}^{0}-\mu_{-2}^{0}=-\ln \left(K_{2}^{\text {theo }}\right)
\end{gathered}
$$

las constantes de equilibrio químico utilizadas en B.3 y B.4 son definiciones teóricas y son las que utilizamos para escribir las expresiones de los grados de disociación luego de la minimización de la energía libre.

Los valores que se encuentran en la bibliografía son aquellos que se obtienen de forma experimental, que se miden de acuerdo con las definiciones dadas previamente en B.1 y B.2. Para relacionar los valores de las constantes de equilibrio experimentales con los valores de las constantes de equilibrio definidas en B.3 y B.4, consideramos las fracciones de disociación para los distintos estados del MEP,

$$
\begin{aligned}
f_{0} & =\frac{\left[\mathrm{PH}_{2}\right]}{\left[\mathrm{PH}_{2}\right]+\left[\mathrm{PH}^{-}\right]+\left[\mathrm{P}^{2-}\right]} \\
f_{-1} & =\frac{\left[\mathrm{PH}^{-}\right]}{\left[\mathrm{PH}_{2}\right]+\left[\mathrm{PH}^{-}\right]+\left[\mathrm{P}^{2-}\right]} \\
f_{-2} & =\frac{\left[\mathrm{P}^{2-}\right]}{\left[\mathrm{PH}_{2}\right]+\left[\mathrm{PH}^{-}\right]+\left[\mathrm{P}^{2-}\right]}
\end{aligned}
$$

dado que las fracciones de disociación involucran las concentraciones de distintas especies, estas expresiones pueden escribirse en términos de las constantes de equilibrio experimentales $K_{1}$ y $K_{2}$, 


$$
\begin{gathered}
f_{0}=\frac{\left[H^{+}\right]^{2}}{\left[H^{+}\right]^{2}+\left[H^{+}\right] K_{1}+K_{1} K_{2}} \\
f_{-1}=\frac{\left[H^{+}\right] K_{1}}{\left[H^{+}\right]^{2}+\left[H^{+}\right] K_{1}+K_{1} K_{2}} \\
f_{-2}=\frac{K_{1} K_{2}}{\left[H^{+}\right]^{2}+\left[H^{+}\right] K_{1}+K_{1} K_{2}}
\end{gathered}
$$

Para hallar la relación entre la definición termodinámica (B.3) y la constante de equilibrio que se obtiene de forma experimental (B.1) se escriben las constantes en relación a los grados de disociación,

$$
K_{1}^{e x p}=\frac{\left[P H^{-}\right]\left[H^{+}\right]}{\left[P H_{2}\right]}=\left[H^{+}\right] \frac{f_{1}}{f_{0}}
$$

y

$$
K_{1}^{\text {theo }}=\frac{\phi_{H^{+}}}{\phi_{\omega}} \frac{f_{1}}{f_{0}}
$$

Si escribimos la última expresión en términos de las propiedades en solución, $\phi_{H^{+}}^{b}=$ $\left[H^{+}\right] 0,602 v_{\omega}$ y $\phi_{\omega}^{b}=1$, y despejamos la fracción $\frac{f_{1}}{f_{0}}$, se obtiene la relación buscada:

$$
K_{1}^{\text {theo }}=0,602 v_{\omega} K_{1}^{\text {exp }}
$$

En términos del $p K=-\log _{10}\left(K_{1}\right)$

$$
p K_{1}^{\text {theo }}=p K^{\text {exp }}-\log _{10}\left(0,602 v_{\omega}\right)
$$

\section{B.0.1. Simetría en las ecuaciones del grado de disociación}

Elegimos escribir siempre los estados de carga neutra como reactivos (a la izquierda del equilibrio químico) y las especies cargadas como productos (a la derecha del equilibrio químico). De esta forma la expresión del grado de disociación tiene siempre la misma estructura y el acoplamiento con protones $\mathrm{H}^{+}$y oxidrilos $\mathrm{OH}^{-}$, es explícita. Por ejemplo,

$$
A H \rightleftharpoons A^{-}+H^{+}, \quad K_{a 1}=\frac{\left[A^{-}\right]\left[H^{+}\right]}{[A H]} \| \quad H_{2} O+B \rightleftharpoons B H^{+}+O H^{-}, \quad K_{b 1}=\frac{\left[B H^{+}\right]\left[O H^{-}\right]}{[B]\left[H_{2} O\right]}
$$

si consideramos que la actividad del agua es igual a uno, la expresión para $f_{1}$ siempre es

$$
\begin{gathered}
f_{a 1}=\frac{\left[A^{-}\right]}{[A H]+\left[A^{-}\right]}=\frac{K_{a 1}}{\left[H^{+}\right]+K_{a 1}} \| f_{b 1}=\frac{\left[B H^{+}\right]}{[B]+\left[B H^{+}\right]}=\frac{K_{b 1}}{\left[O H^{-}\right]+K_{b 1}} \\
f_{b 1}=\frac{\left[e B H^{+}\right]}{[B]+\left[B H^{+}\right]}=\frac{K_{1}}{\left[O H^{-}\right]+K_{1}}
\end{gathered}
$$

Con las últimas expresiones es fácil ver la carga del estado 1 del monómero. Si el acoplamiento es con el ion $\mathrm{OH}^{-}\left(\mathrm{H}^{+}\right)$entonces el monómero está cargado positivamente (negativamente). 
B.1. RELACIONES ENTRE LAS VARIABLES DE BULK EXPERIMENTALES Y LAS VARIABLES DE ENTRADA EN LA TEORÍA APÉNDICE B. SOLUCIONES DE BULK B.1. Relaciones entre las variables de bulk experimentales

\section{y las variables de entrada en la teoría}

La concentración molar de sal en bulk tiene unidades de $\left[C_{\text {salt }}\right]=\frac{m o l}{d m^{3}}$. La fracción de volumen ocupado por los iones debido a la sal se calcula como:

$$
\phi_{\text {ion }}=C_{\text {salt }} \frac{6,02 \times 10^{23} \#}{m o l} \frac{1 d m^{3}}{10^{24} \mathrm{~nm}^{3}}=C_{\text {salt }} \times 0,602 \times v_{\text {ion }}
$$

Para calcular la fracción de volumen ocupada por iones $H^{+}$se utiliza el valor del $\mathrm{pH}$ de la solución:

$$
\phi_{H^{+}}=e^{-p H} \times 0,602 \times v_{H^{+}}
$$




\section{Apéndice $\mathrm{C}$}

\section{Modelo de Capa Polimérica Homogénea}

Para el desarrollo de un modelo sencillo considero una capa homogénea de polímero en contacto con una solución con concentración de sal $C_{\text {salt }}$ y $p H$ fijo. La energía libre para la capa homogénea de polímero se escribe:

$$
\begin{aligned}
\beta F= & \beta \rho_{q} \Psi^{P}+\sum_{i=w, K^{+}, C l^{-}, H^{+}, O H^{-}} \rho_{i}\left[\ln \left(\rho_{i} v_{w}\right)-1+\beta\left(\mu_{i}^{0}-\mu_{i}\right)\right] \\
& +\rho_{P}\left\{\sum_{e=0}^{-2} f_{e}\left[\ln \left(f_{e}\right)+\beta\left(\mu_{e}^{0}-\mu_{H^{+}} n_{e, H^{+}}\right)\right]\right\} \\
& +\beta \pi\left[\rho_{P} v_{p}+\sum_{i=w, K^{+}, C l^{-}, H^{+}, O H^{-}} \rho_{i} v_{i}-1\right]
\end{aligned}
$$

donde $\Psi^{P}$ es el potencial electrostático y $\rho_{q}$ es la densidad de carga en la capa polimérica, $\rho_{i}$ la densidad de las especies presentes en la capa polimérica $i=w, \mathrm{~K}^{+}, \mathrm{Cl}^{-}, \mathrm{H}^{+}, \mathrm{OH}^{-}, \mathrm{P} . \mathrm{f}_{e}$ representa el grado de disociación para el estado $e=0,-1,-2$ y $\mu_{i}$ los potenciales químicos de las especies correspondientes. Como estamos considerando capa polimérica homogénea todas las propiedades son independientes de la posición dentro de la capa polimérica. Conocemos las relaciones

$$
\begin{gathered}
\rho_{q}=\rho_{H^{+}}+\rho_{K^{+}}-\rho_{O H^{-}}-\rho_{C l^{-}}-\rho_{p m e p}\left(f_{-1}+2 f_{-2}\right) \\
\beta \Delta G_{1}=\beta\left(\mu_{-1}^{0}+\mu_{H^{+}}^{0}-\mu_{0}^{0}\right)=-\ln \left(K_{1}\right) \\
\beta \Delta G_{2}=\beta\left(\mu_{-2}^{0}+\mu_{H^{+}}^{0}-\mu_{-1}^{0}\right)=-\ln \left(K_{2}\right)
\end{gathered}
$$

De la minimización de la expresión C.1 se obtienen las expresiones para cada fracción de volumen $\phi_{i}$ :

$$
\beta \mu_{K^{+}}=\ln \left(\phi_{K^{+}}\right)+\beta \Psi^{P}+\beta \pi v_{K^{+}}
$$




$$
\begin{gathered}
\beta \mu_{H^{+}}=\ln \left(\phi_{H^{+}}\right)+\beta \Psi^{P}+\beta \pi v_{H^{+}} \\
\beta \mu_{C l^{-}}=\ln \left(\phi_{C l^{-}}\right)+\beta \Psi^{P}+\beta \pi v_{C l^{-}} \\
\beta \mu_{O H^{-}}=\ln \left(\phi_{O H^{-}}\right)+\beta \Psi^{P}+\beta \pi v_{O H^{-}} \\
0=\ln \left(\phi_{w}\right)+\beta \pi v_{w}
\end{gathered}
$$

Donde se usó que $\mu_{w}=0$, y la expresión para los grados de disociación

$$
\begin{gathered}
\ln \left(\frac{f_{-1}}{f_{0}}\right)=\beta \Psi^{p}+\ln \left(K_{1}\right)-\beta \Delta \mu_{H^{+}} \\
\ln \left(\frac{f_{-2}}{f_{0}}\right)=\beta \Psi^{p}+\ln \left(K_{1} K_{2}\right)-2 \beta \Delta \mu_{H^{+}}
\end{gathered}
$$

Teniendo en cuenta las relaciones de bulk

$$
\begin{gathered}
\ln \left(\rho_{w}^{b} v_{w}\right)=-\beta \pi^{b} v_{w} \\
\beta \mu_{K^{+}}=\ln \left(\rho_{K^{+}}^{b}\right)-\frac{v_{K^{+}}}{v_{w}} \ln \left(\phi_{w}^{b}\right) \\
\beta \mu_{H^{+}}=\ln \left(\rho_{H^{+}}^{b}\right)-\frac{v_{H^{+}}}{v_{w}} \ln \left(\phi_{w}^{b}\right) \\
\beta \mu_{C l^{-}}=\ln \left(\rho_{C l^{-}}^{b}\right)-\frac{v_{C l^{-}}}{v_{w}} \ln \left(\phi_{w}^{b}\right) \\
\beta \mu_{O H^{-}}=\ln \left(\rho_{O H^{-}}^{b}\right)-\frac{v_{O H^{-}}}{v_{w}} \ln \left(\phi_{w}^{b}\right)
\end{gathered}
$$

Podemos escribir:

$$
\begin{gathered}
\ln \left(\frac{\phi_{w}}{\phi_{w}^{b}}\right)=-\beta\left(\pi-\pi^{b}\right) v_{w} \\
\ln \left(\frac{\rho_{K^{+}} v_{w}}{\left(\rho_{w} v_{w}\right)^{v_{k}+v_{w}}}\right)=-\beta \Psi^{p}+\beta \mu_{K^{+}}
\end{gathered}
$$

Redefiniendo todos los potenciales como potenciales químicos de intercambio, y usando las ecuaciones C.2, C.3, C.4 y C.5, podemos escribir:

$$
\begin{aligned}
& \ln \left(K_{1}\right)=\ln \left(\frac{f_{-1}}{f_{0}} \frac{\rho_{K^{+}}}{\rho_{w}^{v_{K}} v_{w}} \frac{\rho_{H^{+}}^{b}}{\rho_{w}^{b}} \frac{\left(\rho_{w}^{b}\right)^{v_{K^{+}} / v_{w}}}{\rho_{K^{+}}^{b}}\right) \\
& \ln \left(K_{2}\right)=\ln \left(\frac{f_{-2}}{f_{-1}} \frac{\rho_{K^{+}}^{v_{K^{+}}} \rho_{w}}{\rho_{H^{+}}^{b}} \frac{\left(\rho_{w}^{b}\right)^{v_{K^{+}} / v_{w}}}{\rho_{w}^{b}}\right)
\end{aligned}
$$

Desde donde derivamos la dependencia de las constantes de equilibrio químico con el estado de carga de los monómeros y la fracción de volumen de polímero, ver ecuaciones C.6 y C.7. 


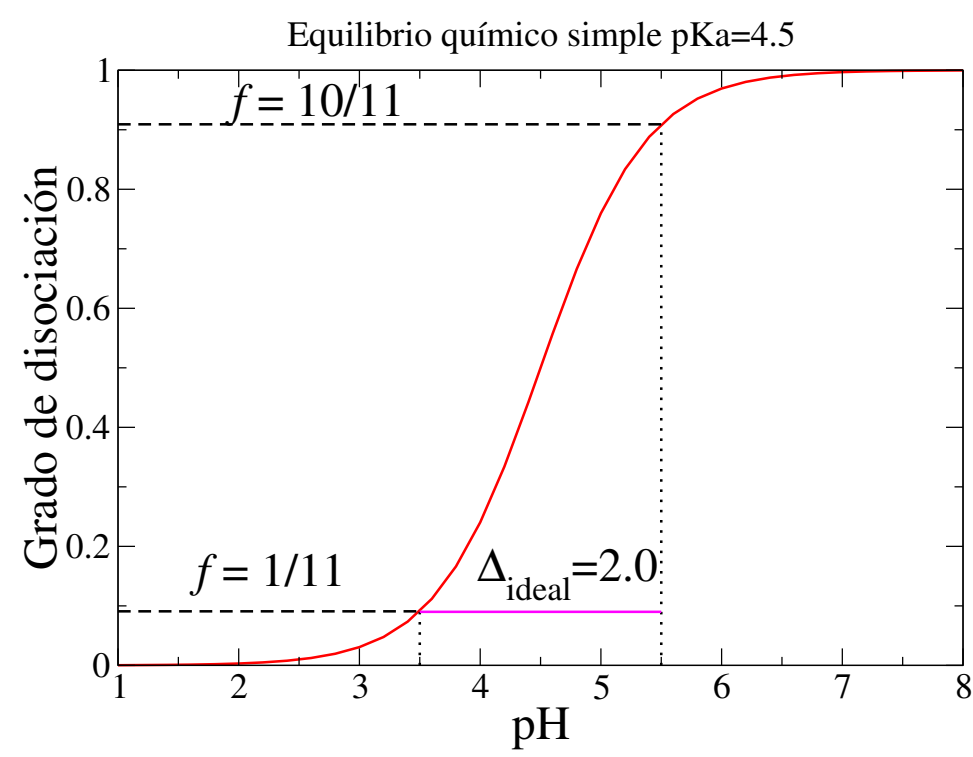

Figura C.0.1: El gráfico muestra una curva de transición ácido-base ideal. Definimos el ancho de la transición $(\Delta)$ como la diferencia de valores de $\mathrm{pH}$ donde el grado de disociación es $1 / 11$ (estado "apagado") y 10/11 (estado encendido). Esta definición asegura $\Delta=2$ para la curva ácido-base ideal.

Para la primera transición aproximamos $f_{-2}=0$, y por lo tanto $f_{0}=1-f_{-1}$. Para la segunda transición aproximamos $f_{0}=0$ y por lo tanto $f_{-1}=1-f_{-2}$. Además usamos las aproximaciones generales: $\phi_{v}^{b}=1$, es decir asumimos que en bulk la solución es aproximadamente agua pura. Dentro del poro el volumen puede estar ocupado por moléculas de agua o monómeros del polímero por ello se asume $\phi_{w}=1-\phi_{p}$. El número de contra iones es igual al número de cargas en la capa polimérica homogénea, es decir pedimos electroneutralidad local.

$$
\begin{aligned}
& p K a_{1}^{a p p}\left(f_{-1}\right)=p K a_{1}^{\text {bulk }}+\log \left(\frac{f_{-1}}{\left(1-f_{-1}\right)}\right)+\log \left(\frac{f_{-1} \phi_{p}}{\left(1-\phi_{p}\right)^{v_{K}+v_{w}}} \frac{v_{w}}{C_{\text {salt }} v_{p}}\right) \\
& p K a_{2}^{a p p}\left(f_{-2}\right)=p K a_{2}^{\text {bulk }}+\log \left(\frac{f_{-2}}{\left(1-f_{-2}\right)}\right)+\log \left(\frac{\left(1+f_{-2}\right) \phi_{p}}{\left(1-\phi_{p}\right)^{v{ }^{+} / v_{w}}} \frac{v_{w}}{C_{\text {salt }} v_{p}}\right)
\end{aligned}
$$

En las últimas expresiones, las constantes de equilibrio de bulk $p K a_{i}^{\text {bulk }}$ con $i=1,2$, corresponden a las definiciones termodinámicas de las constantes de equilibrio de las transiciones; $f_{e}$, con $e=-1,-2$ son los grados de disociación dentro de la interfase polimérica uniforme, $\phi_{P}$ es la fracción de volumen de polímero, $C_{\text {salt }}$ la concentración de sal, $v_{w}, v_{p} \mathrm{y} v_{K^{+}}$son los volúmenes considerados para las distintas especies (ver Cuadro A.3). Estas ecuaciones son importantes por que ofrecen una relación analítica entre las constantes de equilibrio aparentes y la concentración de sal, además predicen correctamente el ancho de las transiciones (ecuaciones (18) y (19) en Gilles et.al[25]). La dependencia de las constantes de equilibrio aparente con la concentración de sal descripta por este modelo analítico es compatible con los valores de $p K a^{a p p}$ que predice la teoría molecular.

Para calcular el ancho de transición del primer equilibrio químico, consideramos los valores de $\mathrm{pH}$ donde $\left\langle f_{-1}\right\rangle=\frac{1}{11} \mathrm{y}\left\langle f_{-1}\right\rangle=\frac{10}{11}$, (ver figuraC.0.1). 


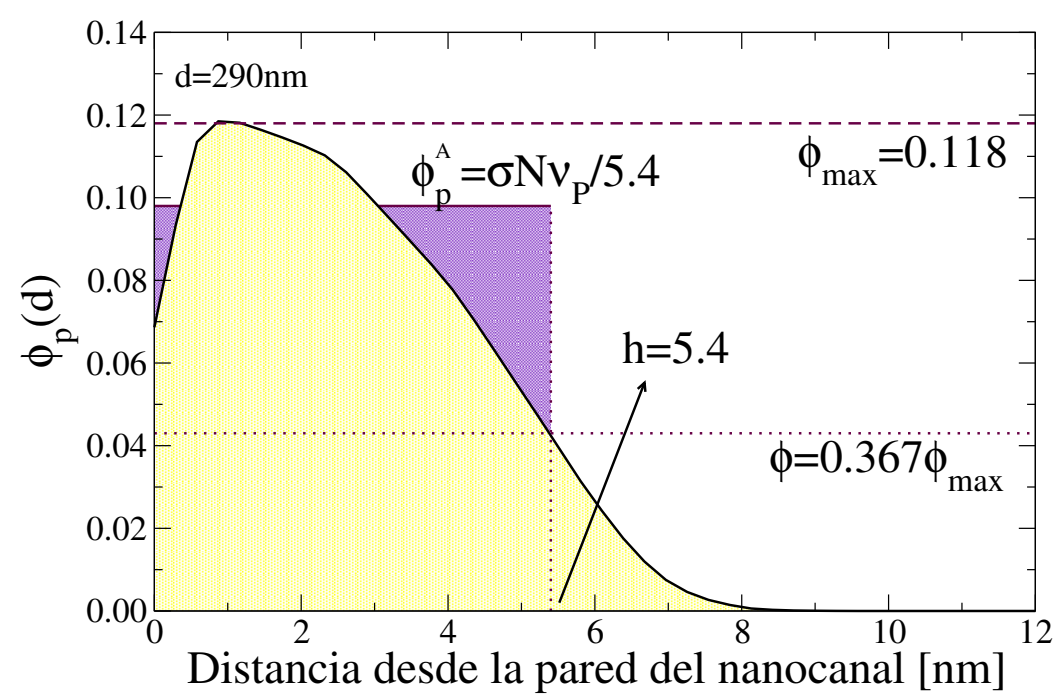

Figura C.0.2: Estimación de la fracción de volumen de la capa polimérica homogénea equivalente a partir de la distribución in-homogénea de polímero que devuelve la teoría molecular.

$$
\begin{aligned}
& p H\left(\left\langle f_{-1}\right\rangle=\frac{1}{11}\right)=p K a_{1}^{\text {bulk }}+\log \left(\frac{\frac{1}{11}}{\left(1-\frac{1}{11}\right)}\right)+\log \left(\frac{\frac{1}{11} \phi_{p}^{A}}{\left(1-\phi_{p}^{A}\right)^{v_{K}{ }^{+/ v_{w}}}} \frac{v_{w}}{C_{s} v_{p}}\right) \\
& p H\left(\left\langle f_{-1}\right\rangle=\frac{10}{11}\right)=p K a_{1}^{\text {bulk }}+\log \left(\frac{\frac{10}{11}}{\left(1-\frac{10}{11}\right)}\right)+\log \left(\frac{\frac{10}{11} \phi_{p}^{B}}{\left(1-\phi_{p}^{B}\right)^{v^{+}{ }^{+/ v_{w}}} \frac{v_{w}}{C_{s} v_{p}}}\right)
\end{aligned}
$$

Donde $\phi_{p}^{A}$ y $\phi_{p}^{B}$ son las fracciones de volumen de polímero cuando $\left\langle f_{-1}\right\rangle=\frac{1}{11} \mathrm{y}\left\langle f_{-1}\right\rangle=\frac{10}{11}$, respectivamente. El ancho de la primera transición está dado por,

$$
p H\left(\left\langle f_{-1}\right\rangle=\frac{10}{11}\right)-p H\left(\left\langle f_{-1}\right\rangle=\frac{1}{11}\right)=2+\log \left(10 \frac{\phi_{p}^{B}}{\phi_{p}^{A}} \frac{\left(1-\phi_{p}^{A}\right)^{v K^{+} / v_{w}}}{\left(1-\phi_{p}^{B}\right)^{v K^{+/ v_{w}}}}\right)
$$

Con el objetivo de estimar el valor de $\phi_{p}^{A}$, determinamos la altura de la capa polimérica $(h=5,4 n m)$ como la distancia desde la pared del nanocanal hasta el punto donde la densidad de monómeros es un $36.7 \%$ del máximo (ver figura C.0.2). Habiendo estimado la altura calculamos la fracción de volumen de la capa homogénea equivalente: $\phi_{p}^{A}=\frac{\sigma N v_{p}}{h}=0,1$

Durante la segunda transición, la densidad y el ancho de la capa polimérica permanecen constantes dado que no hay cambios conformaciones de las cadenas. El ancho de la segunda transición se calcula como,

$$
p K a_{2}^{a p p}\left(\frac{10}{11}\right)-p K a_{2}^{a p p}\left(\frac{1}{11}\right)=2+\log \left(\frac{21}{12}\right)
$$




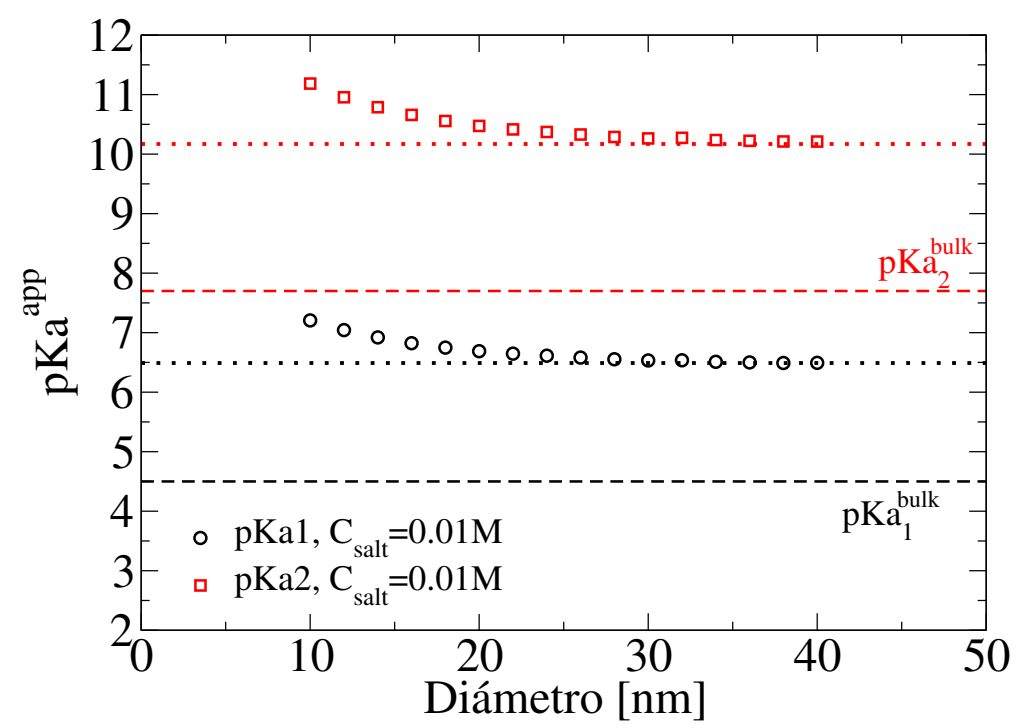

Figura C.0.3: Comparación de los resultados de la teoría molecular con los obtenidos con el modelo simple de capa polimérica homogénea. Con concentración de sal $C_{\text {salt }}=0,01 \mathrm{M}$. 


\section{Apéndice D}

\section{Discretización de las ecuaciones}

\section{D.1. Ecuación de Poisson}

Para la discretización de las ecuaciones se divide la dirección radial en $\operatorname{dim} R$ capas de forma tal que, $r_{j}=\left(j-\frac{1}{2}\right) \delta$ con $j=1, \ldots, \operatorname{dim} R$ y $\delta=\frac{R}{\operatorname{dim} R}=0,5 n m$, ver figura D.0.1. Tomando coordenadas cilíndricas en la ecuación 2.59

$$
\left(\frac{1}{r} \frac{\partial \psi(r)}{\partial r}+\frac{\partial^{2} \psi(r)}{\partial r^{2}}\right)=-\frac{1}{\varepsilon}\left\langle\rho_{q}(r)\right\rangle
$$

Para escribir las ecuaciones discretizadas se calcula la primera derivada como el promedio de los cocientes incrementales por derecha y por izquierda. La derivada segunda se calcula como la diferencia entre los mismos cocientes incrementales,

$$
\begin{gathered}
\frac{\partial \Psi(r)}{\partial r}=\frac{\left(\frac{\partial \Psi}{\partial r}\right)_{d e r}+\left(\frac{\partial \Psi}{\partial r}\right)_{i z q}}{2}=\frac{1}{2 \delta}\left[\Psi\left(r_{j+1}\right)-\Psi\left(r_{j-1}\right)\right] \\
\frac{\partial^{2} \Psi(r)}{\partial r^{2}}=\frac{\left(\frac{\partial \Psi}{\partial r}\right)_{d e r}-\left(\frac{\partial \Psi}{\partial r}\right)_{i z q}}{r_{j+1}-r_{j}}=\frac{1}{\delta^{2}}\left[\Psi\left(r_{j+1}\right)-2 \Psi\left(r_{j}\right)+\Psi\left(r_{j-1}\right)\right]
\end{gathered}
$$

la ecuación de Poisson (2.59) discretizada nos queda,

$$
\psi\left(r_{j+1}\right)-2 \psi\left(r_{j}\right)+\psi\left(r_{j-1}\right)+\frac{1}{2(j-1 / 2)}\left(\psi\left(r_{j+1}\right)-\psi\left(r_{j-1}\right)\right)=-\frac{\delta^{2}}{\varepsilon}\left\langle\rho_{q}\left(r_{j}\right)\right\rangle
$$

donde $r_{j}=(j-1 / 2) \delta$ es el radio evaluado en el centro de la capa $j=1, \ldots, N$. $\varepsilon$ se obtiene en el programa a partir de la longitud de Bjerrum $l_{B}$, según la ecuación:

$$
4 \pi l_{B}=\beta \frac{e^{2}}{\varepsilon_{0} \varepsilon}=\beta \frac{e^{2}}{\varepsilon}
$$

en el código del programa, el potencial electrostático está definido como: $\Psi(r)=\beta e \psi(r)$. Si multiplicamos la expresión D.4 por la magnitud $\beta e$ y usando la definición del potencial nos queda:

$$
\Psi\left(r_{j+1}\right)-2 \Psi\left(r_{j}\right)+\Psi\left(r_{j-1}\right)+\frac{1}{2(j-1 / 2)}\left(\Psi\left(r_{j+1}\right)-\Psi\left(r_{j-1}\right)\right)=-\delta^{2} \frac{\beta e}{\varepsilon} e\left\langle\rho_{q}\left(r_{j}\right)\right\rangle
$$

la segunda carga viene del hecho de que la densidad de carga en el código del programa es 


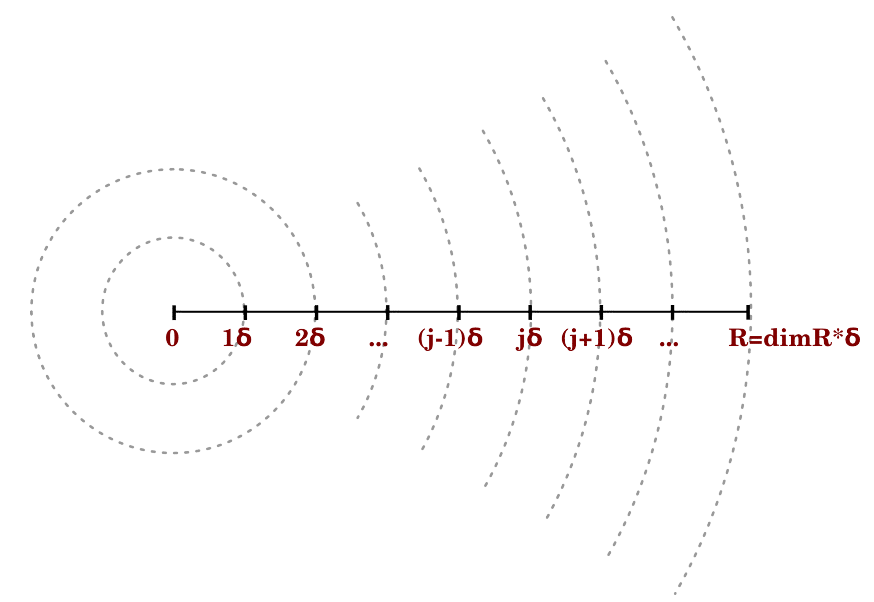

Figura D.0.1: Gráfico de la discretización del volumen del nanocanal. Debido a la simetría cilíndrica sólo se considera la discretización en la dirección radial. Cada capa $j$ tiene sus bordes entre $(j-1) \delta$ y $j \delta$, con $j=1, \ldots, \operatorname{dim} R$.

por unidad de carga por lo que nunca fue multiplicada por la carga fundamental. Por lo tanto la expresión D.5, incorpora naturalmente la longitud de Bjerrum.

$$
\Psi\left(r_{j+1}\right)-2 \Psi\left(r_{j}\right)+\Psi\left(r_{j-1}\right)+\frac{1}{2(j-1 / 2)}\left(\Psi\left(r_{j+1}\right)-\Psi\left(r_{j-1}\right)\right)=-\delta^{2}\left(4 \pi l_{b}\right)\left\langle\rho_{q}\left(r_{j}\right)\right\rangle
$$

\section{Condiciones de contorno}

En el centro del canal por simetría axial el campo electrostático debe ser cero (no hay ninguna dirección privilegiada). Por lo tanto es apropiada la condición de contorno:

$$
\frac{\partial \Psi(0)}{\partial r}(r=0)=0
$$

En cambio sobre la pared del nanocanal puede haber una distribución de carga que asumimos homogénea en la dirección angular $\hat{\theta}$. Por lo tanto el flujo de campo $E$ a través de esta superficie se calcula utilizando la ley de Gauss:

$$
E_{2}-E_{1}=\frac{\sigma_{q}}{\varepsilon}
$$

donde 2 es dentro de la membrana polimérica y 1 es dentro del nanocanal. Como en el seno de la membrana no hay cargas y el potencial electrostático en el bulk debe converger a un potencial constante, el campo eléctrico en 2 debe ser cero . $E_{2}=0$. Además como $E_{1}=-\frac{\partial \Psi}{\partial r}=$ $-\frac{\Psi_{N}-\Psi_{N-1}}{\delta}$

$$
E_{1}=-\frac{\sigma_{q}}{\varepsilon}=-\frac{\Psi_{N}-\Psi_{N-1}}{\delta}
$$

y por lo tanto nuestra condición de contorno es

$$
\frac{\partial \Psi(R)}{\partial r}(r=R)=\frac{\sigma_{q}}{\varepsilon}
$$

En sus formas discretizadas las condiciones de contorno D.7 y D.8 se escriben,

$$
\Psi(0)=\Psi(1)
$$




$$
\Psi\left(r_{N}\right)=\Psi\left(r_{N-1}\right)+\delta \frac{\sigma_{q}}{\varepsilon}
$$

estas condiciones de contorno fueron testeadas con el modelo teórico descripto por Schoch[56] y resultan adecuadas para la descripción del potencial electrostático. Acomodando las unidades como en D.6 la condición de contorno sobre la pared del nanocanal queda,

$$
\Psi\left(r_{N}\right)=\Psi\left(r_{N-1}\right)+\delta\left(4 \pi l_{b}\right) \sigma_{q}
$$

\section{D.2. Discretización de la función densidad de probabili- dad $P(\alpha)$}

La ecuación 2.58 para la probabilidad de una conformación polimérica debe ser discretizada para ser resuelta computacionalmente. Antes de comenzar con la discretización de la ecuación de $P(\alpha) 2.58$, reemplazamos las expresiones de los grados de disociación obtenidas al minimizar la funcional ecuación 2.47. Obtenemos:

$$
P(\alpha)=\frac{1}{Q} \exp \left(-\int_{0}^{R} n(r ; \alpha) \times\left\{\beta \pi(r) v_{p}+\ln \left(f_{0}(r)\right)-\beta \chi_{\text {wall }}(r)\right\}\left(\frac{r}{R}\right) d r\right)
$$

la idea es calcular $P(\alpha)$ como el producto de las probabilidad de Boltzmann de tener un segmento en una capa dada, debido a la conformación $\alpha$.

$$
P(\alpha)=\frac{1}{Q} \prod_{j=1} p\left(r_{j}\right)^{N_{\left(r_{j} ; \alpha\right)}}
$$

Es importante observar que $N_{\left(r_{j} ; \alpha\right)}$ es el número de monómeros en la capa $r_{j}, r_{j}+d r$ debido a la conformación $\alpha$ y este número tiene en cuenta el jacobiano de la integral.

$$
N_{\left(r_{j}, \alpha\right)}=n\left(r_{j} ; \alpha\right) \frac{r_{j}}{\operatorname{dim} R}
$$

La probabilidad de la capa se calculada como:

$$
p\left(r_{j}\right)=e^{-\left(\beta \pi\left(r_{j}\right) v_{p}+\ln \left(f_{0}\left(r_{j}\right)\right)-\beta \chi_{w a l l}\left(r_{j}\right)\right)}
$$

acomodando la expresión nos queda,

$$
p\left(r_{j}\right)=e^{\beta \chi_{w a l l}\left(r_{j}\right)} \frac{1}{f_{0}\left(r_{j}\right) \phi_{w}\left(r_{j}\right)^{v_{p} / v_{w}}}
$$




\section{Apéndice E}

\section{Aproximación de Campo Medio}

A continuación se explica la aproximación de campo medio que permite desacoplar la probabilidad de la conformación de una dada cadena de la conformación de las cadenas vecinas. Esto se hace reemplazando la interacción con las cadenas vecinas por un campo promedio. Para realizar la aproximación escribimos la densidad de monómeros en función de la distancia a una superficie plana,

$$
n_{m}(z)=\sum_{\{\alpha\}} P(\{\alpha\}) n_{m}(z ; \alpha)
$$

donde $\sum_{\{\alpha\}}$ significa la suma sobre todos los posibles estados de cada conformación. $n_{m}(z)$ densidad de monómeros en $z, n_{m}(z ; \alpha)$ densidad de monómeros en $z$ debido a la configuración $\alpha$. Supongamos que se generan con el modelo de RIS dos millones de conformaciones de cadenas. La expresión E.1, implica hacer la suma para cada cadena sobre los dos millones de conformaciones. Si el sistema tiene $N_{p}$ cadenas poliméricas entonces el símbolo $\sum_{\{\alpha\}}$ consiste en $N_{p}$ sumatorias $\sum_{\alpha_{1}} \sum_{\alpha_{2}} \ldots \sum_{\alpha_{N_{p}}}$, donde $\alpha$ etiqueta cada conformación de cadena y va desde uno hasta dos millones. Es posible reescribir la función de probabilidad del conjunto de las cadenas $P(\{\alpha\})=$ $P\left(\alpha_{1}, \alpha_{2}, \ldots, \alpha_{N_{p}}\right)$ bajo la aproximación de campo medio como

$$
P(\{\alpha\})=P\left(\alpha_{1}\right) P\left(\alpha_{2}\right) \ldots P\left(\alpha_{N_{p}}\right)
$$

donde se asume que la probabilidad de la conformación de una dada cadena es independiente del resto de las cadenas. Usando la última expresión en la ecuación E.1, se obtiene

$$
\begin{aligned}
n_{m}(z) & =\left(\sum_{\alpha_{1}} P\left(\alpha_{1}\right) n_{m}\left(z ; \alpha_{1}\right)\right)\left(\sum_{\alpha_{2}} P\left(\alpha_{2}\right)\right)\left(\sum_{\alpha_{3}} P\left(\alpha_{3}\right)\right) \ldots\left(\sum_{\alpha_{N_{p}}} P\left(\alpha_{N_{p}}\right)\right) \\
& +\left(\sum_{\alpha_{1}} P\left(\alpha_{1}\right)\right)\left(\sum_{\alpha_{2}} P\left(\alpha_{2}\right) n_{m}\left(z ; \alpha_{2}\right)\right)\left(\sum_{\alpha_{3}} P\left(\alpha_{3}\right)\right) \ldots\left(\sum_{\alpha_{N_{p}}} P\left(\alpha_{N_{p}}\right)\right) \\
& +\left(\sum_{\alpha_{1}} P\left(\alpha_{1}\right)\right)\left(\sum_{\alpha_{2}} P\left(\alpha_{2}\right)\right)\left(\sum_{\alpha_{3}} P\left(\alpha_{3}\right) n_{m}\left(z ; \alpha_{3}\right)\right) \ldots\left(\sum_{\alpha_{N_{p}}} P\left(\alpha_{N_{p}}\right)\right) \\
+\ldots+ & \left(\sum_{\alpha_{1}} P\left(\alpha_{1}\right)\right)\left(\sum_{\alpha_{2}} P\left(\alpha_{2}\right)\right)\left(\sum_{\alpha_{3}} P\left(\alpha_{3}\right)\right) \ldots\left(\sum_{\alpha_{N_{p}}} P\left(\alpha_{N_{p}}\right) n_{m}\left(z ; \alpha_{N_{p}}\right)\right)
\end{aligned}
$$


cada término con la forma $\left(\sum_{\alpha_{i}} P\left(\alpha_{i}\right)\right)$ es igual a $1\left(\left(\sum_{\alpha_{1}} P\left(\alpha_{i}\right)\right)=1\right)$ debido a la condición de normalización, usando esta propiedad y usando que las cadenas poliméricas son similares unas a otras (indistinguibles entre si).

$$
n_{m}(z)=N_{p}\left(\sum_{\alpha} P(\alpha) n_{m}(z ; \alpha)\right)
$$

donde $\alpha$ itera sobre todas las conformaciones de cadena generadas.

Consideremos una geometría cilíndrica donde incorporamos la posibilidad de traslación para las cadenas poliméricas. En este caso, la aproximación cambia un poco. Ahora la densidad de monómeros en $r$ dependerá de la conformación de cadenas etiquetadas con un índice discreto $\alpha$ y un índice continuo $r^{\prime}$ que integra sobre todas las posibles posiciones espaciales de la conformación de cadenas en coordenadas cilíndricas. Como asumimos homogeneidad en la dirección $\hat{\theta}$ esa integral devuelve un factor $2 \pi$.

$$
n_{m}(r)=\sum_{\left\{r^{\prime}, \alpha\right\}} P\left(\left\{r^{\prime}, \alpha\right\}\right) n_{m}\left(r ;\left\{r^{\prime}, \alpha\right\}\right)
$$

donde $\sum_{\left\{r^{\prime}, \alpha\right\}}$ significa la suma sobre todos los posibles estados de cada conformación $\left(r^{\prime}, \alpha\right)$. Siguiendo el ejemplo anterior si el sistema tiene $N_{p}$ cadenas poliméricas entonces el símbolo $\sum_{\left\{r^{\prime}, \alpha\right\}}$ consiste en $N_{p}$ sumatorias $\left(\sum_{\alpha_{1}} \int_{0}^{R} 2 \pi r_{1}^{\prime} d r_{1}^{\prime}\right)\left(\sum_{\alpha_{2}} \int_{0}^{R} 2 \pi r_{2}^{\prime} d r_{2}^{\prime}\right) \ldots\left(\sum_{\alpha_{N_{p}}} \int_{0}^{R} 2 \pi r_{N_{p}}^{\prime} d r_{N_{p}}^{\prime}\right)$. Reescribiendo la expresión E.2, y forzando la condición de normalización local de la probabilidad de cadena $\sum_{\alpha} P\left(r^{\prime}, \alpha\right)=1$, se ve que cada término aporta $N_{p}\left(\pi R^{2}\right)$ a la densidad de monómeros en $r$

$$
n_{m}(r)=\frac{N_{P}}{\pi R^{2}}\left(\pi R^{2}\right)^{N_{p}}\left(\sum_{\alpha} \int_{0}^{R} P\left(r^{\prime}, \alpha\right) n_{m}\left(r ; r^{\prime}, \alpha\right)\left(2 \pi r^{\prime}\right) d r^{\prime}\right)
$$

si el número de monómeros por cadena es $N_{\text {long }}$ entonces

$$
2 \pi \int_{0}^{R} n_{m}(r) r d r=N_{p} \times N_{\text {long }}
$$


$n\left(r ; r^{\prime}, \alpha\right)$ Sobre la definición de esta magnitud:

Las propiedades de $n\left(r ; r^{\prime}, \alpha\right)$ son tres:

1. La suma sobre todas las posición $r$, debería dar el número total de monómeros de la cadena polimérica $\left(r^{\prime}, \alpha\right)$ que será $N_{m o n}$ por que considero todas las cadenas igual de largas.

$$
\int_{0}^{R} n\left(r ; r^{\prime}, \alpha\right) r d r=\frac{N_{m o n}\left(r^{\prime}, \alpha\right)}{2 \pi L}=\frac{N_{m o n}}{2 \pi L}
$$

2. La suma sobre la variable continua de la conformación $\boldsymbol{r}^{\prime}$, es el número total de monómeros en $r$, debido a todas las conformaciones $\alpha$. (cómo si estuviera la misma conformación $\alpha$ en todas las posiciones)

$$
\int_{0}^{R} n\left(r ; r^{\prime}, \alpha\right)\left(2 \pi r^{\prime}\right) d r^{\prime}=n(r ; \alpha)
$$

3. Entonces

$$
\int_{0}^{R}\left(\int_{0}^{R} n\left(r ; r^{\prime}, \alpha\right) r d r\right)\left(2 \pi r^{\prime}\right) d r^{\prime}=\frac{N_{m o n}}{2 \pi L}\left(\pi R^{2} L\right)=N_{m o n} \frac{R^{2}}{2}
$$

4. La suma sobre la variable discreta de las conformaciones $\alpha$, es el número de monómeros en $r$ debido a todas las conformaciones $\{\alpha\}$ posicionadas en $r^{\prime}$

$$
\sum_{\alpha} n\left(r ; r^{\prime}, \alpha\right)=n\left(r ; r^{\prime}\right)
$$

5. Colapsando todos los índices

$$
\sum_{\alpha} \int_{0}^{R}\left(\int_{0}^{R} n\left(r ; r^{\prime}, \alpha\right) r d r\right)\left(2 \pi r^{\prime}\right) d r^{\prime}=\frac{N_{m o n}}{2 \pi L}\left(\pi R^{2} L\right) N_{\alpha^{\prime} s}
$$

En el código del programa utilizado la magnitud $n\left(r ; r^{\prime}, \alpha\right)$ se redefine para contener el jacobiano de las coordenadas: $n\left(r ; r^{\prime}, \alpha\right)=r r^{\prime} n\left(r ; r^{\prime}, \alpha\right)$. 


\section{Apéndice F}

\section{Método Variacional}

Dada la funcional $S$ dependiente de las variables independientes $q$ y sus derivadas primeras $\dot{q}$,

$$
S=\int L(q, \dot{q}, t) d t
$$

El mínimo de la función $S(\delta S=0)$ implica las ecuaciones de Euler-Lagrange para la función $L(q, \dot{q}, t):$

$$
\frac{d L(q, \dot{q}, t)}{d q}-\frac{d}{d t}\left(\frac{\delta L(q, \dot{q}, t)}{\delta \dot{q}}\right)=0
$$

La optimización de la funcional $\omega$, que describe nuestro sistema, consiste en utilizar las ecuaciones de Euler-Lagrange. La ecuación 2.33 es una ecuación funcional que, salvo la derivada del potencial electrostático, no depende de la primer derivada del resto de las variables independientes, es decir: $\omega=\omega(q, t)$. En la teoría molecular que se presenta las variables independientes son: $P(\alpha), \rho_{i}(r)$ con $i=C l^{-}, O H^{-}, K^{+}, H^{+}, f_{e}(r)$ con $e=1, . ., m$ y $\Psi(r)$.

Por lo tanto,

$$
\omega=\int W\left(P(\alpha), \rho_{C l^{-}}(\boldsymbol{r}), \rho_{K^{+}}(\boldsymbol{r}), \rho_{O H^{-}}(\boldsymbol{r}), \rho_{H^{+}}(\boldsymbol{r}), f_{e}(\boldsymbol{r}), \Psi(\boldsymbol{r}), \nabla_{r} \Psi(\boldsymbol{r}), \boldsymbol{r}\right) d \boldsymbol{r}
$$

se pueden hallar sus puntos estacionarios utilizando la ecuación F.2,

$$
\begin{array}{lll}
\frac{d W}{d P(\alpha)} & = & 0 \\
\frac{d W}{d \rho_{i}(r)} & = & 0 \\
\frac{d W}{d f_{e}(r)} & = & 0 \\
\frac{d W}{d \Psi(r)}- & \frac{d}{d r}\left(\frac{\delta W}{\delta \Psi(r)}\right)= & 0
\end{array}
$$

Si bien los mínimos, máximos y puntos de ensilladura cumplen estas condiciones, nosotros sabemos (empíricamente) que nuestros métodos numéricos localizan los mínimos locales. 